ṽ

0
0
$\omega$
$w$

BIBLIOTHEEK TU Delft

P 13014035

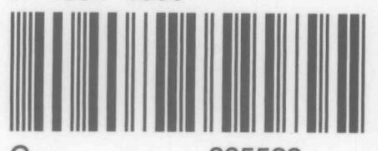

C 

ON NON-LINEAR

DISPERSIVE WATER WAVES 



\section{ON NON-LINEAR \\ DISPERSIVE WATER WAVES}

PROEFSCHRIFT

TER VERKRIJGING VAN DE GRAAD VAN DOCTOR IN DE TECHNISCHE WETENSCHAPPEN AAN DE TECHNISCHE HOGESCHOOL DELFT, OP GEZAG VAN DE RECTOR MAGNIFICUS DR. IR. C. J. D. M. VERHAGEN, HOOGLERAAR IN DE AFDELING DER TECHNISCHE NATUURKUNDE, VOOR EEN COMMISSIE UIT DE SENAAT TE VERDEDIGEN OP WOENSDAG 11 DECEMBER 1968 TE 14 UUR

DOOR

\section{HENDRIK WILLEM HOOGSTRATEN wiskundig ingenieur geboren te Leiden}

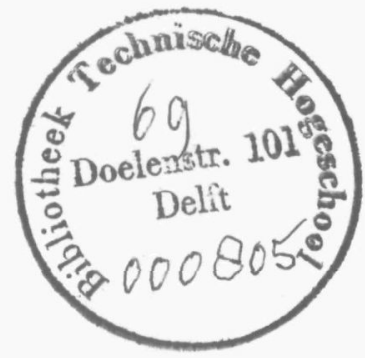




$$
-
$$





\section{CONTENTS}

INTRODUCTION. . . . . . . . . . . . . . . . . . . . . . 7

1 Dispersion of waves . . . . . . . . . . . . . . . . . . . 7

2 Linear waves . . . . . . . . . . . . . . . . 9

3 Whitham's theory for non-linear conservative dispersive waves . 11

4 A general asymptotic theory of non-linear slowly varying wavetrains . . . . . . . . . . . . . 13

Chapter I CNOIDAL WAVES

I.1 The Korteweg-de Vries equation . . . . . . . . . . . . . . 16

I.2 Asymptotic expansion with respect to K . . . . . . . . . . . 17

I.3 Two integral relations for $U(p, x, t)$. . . . . . . . . . . . . 20

I.4 Conservation equations for cnoidal waves . . . . . . . . . . 21

I.5 Asymptotic expansion of $U(p, x, t)$ in powers of $\varepsilon$. . . . . . . 23

I.6 The equations for $\kappa, \omega, E$ and $H_{0}$. . . . . . . . . . . . . . . . . . 28

I.7 An asymptotic solution of the initial value problem for slowly varying wavetrains . . . . . . . . . . . . . . . 31

Chapter II BOUSSINESQ WAVES

II.1 The Boussinesq equations . . . . . . . . . . . . . . . . . 35

II.2 Asymptotic expansions with respect to $K$ for the one-dimensional case . . . . . . . . . . . . . . . . . . . . 37

II.3 Three integral relations involving $U(p, x, t)$ and $H(p, x, t)$. . . 39

II.4 Conservation laws for one-dimensional Boussinesq waves . . . 41

II.5 Asymptotic expansion of $U(p, x, t)$ and $H(p, x, t)$ in powers of $\varepsilon 43$

II.6 Reduction of the integral relations . . . . . . . . . . . . . 46

II.7 Investigation of the equations for $\kappa, \omega, E, H_{0}$ and $U_{0} \ldots \ldots$. . 48

II.8 Two-dimensional Boussinesq waves . . . . . . . . . . . . . 52

II.9 Conservative equations and integral relations for two-dimensional Boussinesq waves. . . . . . . . . . . . . . . . . . 57

\section{Chapter III STOKES WAVES}

III.1 Formulation of the problem and the asymptotic representation of a slowly varying wavetrain. . . . . . . . . . . . . . . 61

III.2 Investigation of the boundary value problem for $\chi(p, x, y, t)$,

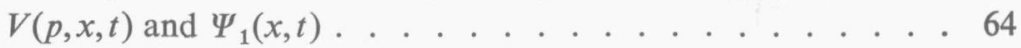

Appendix . . . . . . . . . . . . . . . . . . . . 71

References . . . . . . . . . . . . . . . . . 79

Samenvatting . . . . . . . . . . . . . . . . . . 81 


\section{INTRODUCTION}

\section{Dispersion of waves}

In this thesis we study the propagation of three kinds of non-linear dispersive water waves, viz. cnoidal waves, Boussinesq waves and Stokes waves. At first we give a survey of linear and non-linear dispersive wave phenomena.

Consider a one-dimensional function $\eta(x, t)$ depending on one space coordinate $x$ and on the time $t$ and which satisfies a partial differential equation in $x$ and $t$. A solution $\eta(x, t)$ of this partial differential equation is said to represent a one-dimensional uniform wave propagating in the direction of the positive $x$-axis if it is a function of a single phase coordinate $\theta=\kappa x-\omega t$ :

$$
\eta(x, t)=\eta(\kappa x-\omega t), \quad \kappa>0, \quad \omega>0 .
$$

The constants $\kappa$ and $\omega$ are called the wavenumber and the frequency respectively. The phase velocity $c$ defined by $c=\omega / \kappa$, may be considered as the velocity with which a 'point' of constant phase moves in the direction of the positive $x$-axis. In the $x, t$-plane the curves of constant phase are called equi-phase lines or wavefronts. For the uniform wave (1) these curves are straight lines.

The description of a one-dimensional wave phenomenon by means of a single wavefunction $\eta(x, t)$ is by no means a general one. It appears that in some cases a set of $m$ wavefunctions $\eta_{1}(x, t), \eta_{2}(x, t), \ldots, \eta_{m}(x, t)$ is needed to describe a wave phenomenon. A uniform progressive wave is then defined by taking all functions $\eta_{1}(x, t)$, $\eta_{2}(x, t), \ldots, \eta_{m}(x, t)$ as functions of the same phase coordinate $\theta=\kappa x-\omega t$. This case will be encountered in Chapter II dealing with Boussinesq waves.

A more complicated case is treated in Chapter III on Stokes waves. In that case a partial differential equation has to be satisfied with boundary conditions imposed on a wave-like curve that has to be determined as a part of the problem. Also we may have $n$-dimensional wavefunctions $\eta\left(x_{1}, x_{2}, \ldots, x_{n}, t\right)$ for which the uniform (plane) wave is defined by taking $\eta$ as a function of the $n$-dimensional phase coordinate

$$
\theta=\kappa_{1} x_{1}+\kappa_{2} x_{2}+\ldots+\kappa_{n} x_{n}-\omega t
$$

However, in the survey given in this Introduction we restrict ourselves to onedimensional wave phenomena that can be described by a single wavefunction $\eta(x, t)$. This case is dealt with in Chapter I on cnoidal waves.

The dependence of the wavefunction $\eta$ on the phase coordinate $\theta$ in the case of a 
uniform propagating wave is found by substitution of equation (1) into the governing partial differential equation for $\eta(x, t)$. It is evident that only a restricted class of partial differential equations has solutions of the form of a uniform propagating wave $\eta=\eta(\kappa x-\omega t)$. A general criterion whether a partial differential equation possesses such wave-like solutions is not available and in this thesis we only consider problems for which the uniform propagating wave solution exists. But even when substitution of (1) into the basic partial differential equation leads to an ordinary differential equation for $\eta(\theta)$, this still does not mean that the problem has solutions representing a uniform periodic wave. If $\eta(\theta)$ is not periodic in $\theta$ one usually speaks of 'waves', (we mention shock waves and solitary waves) but notions like wavelength, frequency etc. loose their significance. In the following we will always consider problems for which the uniform propagating wave solution exists and has the form of a bounded periodic function $\eta(\theta)$ of the phase coordinate $\theta=\kappa x-\omega t$. We call such a solution a uniform wavetrain.

Depending on the order of the ordinary differential equation for $\eta(\theta)$ the uniform wavetrain solution may depend on a number of integration constants $\alpha_{1}, \alpha_{2}, \ldots, \alpha_{m}$ :

$$
\eta(x, t)=\eta\left(\kappa x-\omega t ; \alpha_{1}, \alpha_{2}, \ldots, \alpha_{m}\right) .
$$

Mostly these additional constants can be identified with quantities of physical interest such as amplitude, mean waveheight, etc.

By defining the phase function $\theta$ as $\kappa x-\omega t$ the wavenumber $\kappa$ is not fixed uniquely. The period of $\eta(\theta)$ is in general a function of the parameters $\kappa, \omega, \alpha_{1}, \ldots, \alpha_{m}$ and may be normalized to one or $2 \pi$. This gives a relation between the $m+2$ constants:

$$
F\left(\kappa, \omega, \alpha_{1}, \alpha_{2}, \ldots, \alpha_{m}\right)=0,
$$

which is called the dispersion relation. Waves for which the dispersion relation reduces to

$$
c=\frac{\omega}{\kappa}=\text { constant }
$$

are called non-dispersive: the phase velocity $c$ for uniform wavetrains with different wavenumbers is equal. For instance when the governing partial differential equation is linear, a wave composed of a linear superposition of non-dispersive uniform wavetrains moves without distortion and with constant phase velocity $c$ in the direction of the positive $x$-axis.

All other waves which are accordingly called dispersive waves, behave in a different way. A general wavetrain of dispersive waves will have a continuously changing shape and velocity and the only waves that may propagate without distortion and 
with constant velocity are the uniform periodic wavetrains given by equation (2). Possible exceptions such as the solitary wave which is the limiting case for infinite wavelength will not be considered here.

Dispersion is also the cause of the phenomenon that a dispersive wave system initiated by an arbitrary disturbance will develop after a considerable time into a socalled slowly varying wavetrain. A slowly varying wavetrain is a wavetrain for which typical quantities such as wavenumber, wavelength, amplitude, etc. change only by a very small fraction of themselves during one period or within one wavelength. Such waves that locally may be considered as nearly uniform are the object of investigation in this thesis. The expression 'slowly varying' refers to the two fundamental scales which are important for the description of these wavetrains: a 'microscopic' scale in which the local oscillations are nearly periodic and a large 'macroscopic' scale in which quantities like wavenumber, frequency, amplitude, etc. may change considerably. The asymptotic theory used in the sequel is based on the separation of these two scales for slowly varying wavetrains. This will enable us to gain insight into the large-scale variations of the wavetrain which are of physical interest separated from the local periodic oscillatory behaviour.

In the next section of this Introduction we give a brief survey of linear waves, i.e. wave phenomena governed by linear partial differential equations with linear boundary conditions and show that for equations with constant coefficients indeed an initial disturbance disperses into a slowly varying wavetrain. Furthermore a direct asymptotic method for linear dispersive waves, viz. the ray theory, is mentioned. In section 3 we discuss the theories of WhITHAM and LighTHILL that have been developed in recent years in order to deal with non-linear wavetrains. The last section is devoted to an exposition of the general asymptotic theory for non-linear waves which forms the starting point for the subsequent chapters dealing with non-linear water waves.

\section{Linear waves}

For wave phenomena governed by linear partial differential equations with constant coefficients it is a well-known fact that the uniform progressive wavetrain is sinusoidal and that the dispersion relation only contains the wavenumber $\kappa$ and the frequency $\omega$. In the complex notation the uniform wavetrain propagating in the direction of the positive $x$-axis is given by:

$$
\eta(x, t)=a \exp [i\{\kappa x-\omega(\kappa) t\}], \quad \omega=\omega(\kappa) .
$$

It is typical of linear waves that the amplitude $a$ may be chosen arbitrarily without influencing the frequency $\omega$.

According to the superposition principle for linear problems any arbitrary wave- 
train can be decomposed in its harmonic components and hence it is possible to give an exact solution of linear wave equations with constant coefficients by means of a Fourier integral:

$$
\eta(x, t)=\int_{0}^{\infty} f(\kappa) \exp [i\{\kappa x-\omega(\kappa) t\}] d \kappa .
$$

Although this is an explicit exact solution many properties of it remain obscure and much more information may be obtained from it after an asymptotic expansion for large $x$ and $t$ by means of the method of stationary phase:

$$
\eta(x, t) \sim\left\{\frac{2 \pi}{t\left|\omega^{\prime \prime}\left(\kappa_{o}\right)\right|}\right\}^{\frac{1}{2}} f\left(\kappa_{0}\right) \exp \left[i\left\{\kappa_{0} x-\omega\left(\kappa_{0}\right) t\right\}-\frac{\pi i}{4} \operatorname{sgn} \omega^{\prime \prime}\left(\kappa_{0}\right)\right], \underset{\left(\omega^{\prime \prime}\left(\kappa_{0}\right) \neq 0\right),}{,}
$$

where $\kappa_{0}(x, t)$ is to be determined from the equation

$$
\frac{x}{t}=\left[\frac{d \omega}{d \kappa}\right]_{\kappa=\kappa_{0}(x, t)} .
$$

It is observed that asymptotic expansion (4) indeed represents a slowly varying wavetrain. The exponential function oscillates with wavelength and period of order unity, whereas the local wavenumber $\kappa_{0}(x, t)$ is a slowly varying function of $x$ and $t$ which can be seen from the relations

$$
\begin{aligned}
& \frac{1}{\kappa_{0}} \frac{\partial \kappa_{0}}{\partial x}=\frac{1}{t \kappa_{0} \omega^{\prime \prime}\left(\kappa_{0}\right)}=O\left(\frac{1}{t}\right), \\
& \frac{1}{\kappa_{0}} \frac{\partial \kappa_{0}}{\partial t}=-\frac{\omega^{\prime}\left(\kappa_{0}\right)}{t \kappa_{0} \omega^{\prime \prime}\left(\kappa_{0}\right)}=O\left(\frac{1}{t}\right),
\end{aligned}
$$

following from equation (5).

The quantity $\omega^{\prime}(\kappa)$ has the dimension of a velocity and is called the group velocity. It may be shown (BRoER [1], LighthiLl [2]) that the energy transported by a conservative linear wavetrain is propagated with group velocity. For further information we refer to ECKART [3], BriLlouin \& SOMMERFELD [4] and JeFFreys \& JeFFreys [5].

A direct asymptotic method is provided by the ray theory developed by KELLER which also may be applied to linear equations with variable coefficients. Although in that case a uniform wavetrain does not exist, a slowly varying wavetrain solution is expected to be possible if the coefficients in the basic equation are slowly varying functions of $x$ and $t$. Under these conditions an asymptotic expansion of a linear 
slowly varying wavetrain with wavelength of order of magnitude $K^{-1}$ (with $K \gg 1$ ) is expected to be of the form:

$$
\eta(x, t)=\left[A_{0}(x, t)+\frac{1}{i K} A_{1}(x, t)+\frac{1}{(i K)^{2}} A_{2}(x, t)+\ldots\right] e^{i K S(x, t)}
$$

This expansion is inserted into the governing equation and the coefficients of the various powers of $K$ are equated to zero. Then the coefficient of the highest power of $K$ yields a first order partial differential equation for the phase function $S(x, t)$ of which the characteristics are called 'rays'. The coefficients of the subsequent powers of $K$ lead to 'transport equations' for the amplitude functions $A_{0}(x, t), A_{1}(x, t), \ldots$, which reduce to ordinary differential equations along the rays. Further details can be found in an extensive paper of LEwIS [6]. In this connection we also mention articles of Bleistein \& Lewis [7] and Boersma [8].

\section{Whitham's theory for non-linear conservative dispersive waves}

In recent years a theory for non-linear conservative wave problems has been developed by Whitham $[9,1011,12]$ and refined for a restricted class of non-linear problems by LighthiLL $[13,14]$. The basic assumption in Whitham's theory is that a non-linear slowly varying wavetrain may be represented locally, i.e. within a small number of wavelengths and during a small number of periods, by the uniform wavetrain solution (2) with slowly varying parameters $\kappa(x, t), \omega(x, t), \alpha_{1}(x, t), \ldots, \alpha_{m}(x, t)$. The problem is then to find a set of $m+2$ equations for these $m+2$ unknown functions governing the large-scale variations of the wavetrain.

In Whiтнам [9] an averaging technique is applied to $m+2$ conservative equations which may be obtained for a conservative wave problem. These conservation laws have the form:

$$
\frac{\partial P_{i}(x, t)}{\partial t}+\frac{\partial Q_{i}(x, t)}{\partial x}=0, \quad i=1,2,3, \ldots, m+2 .
$$

Next for the uniform wavetrain solution (2) of the problem the quantities $P_{i}(x, t)$ and $Q_{i}(x, t)$ are averaged over one period. The mean values, denoted by $\bar{P}_{i}$ and $\bar{Q}_{i}$, are functions of the $m+2$ parameters $\kappa, \omega, \alpha_{1}, \ldots, \alpha_{m}$ and in the case of a slowly varying wavetrain these $m+2$ parameters are considered as slowly varying functions of $x$ and $t$. The $m+2$ equations determining these functions are then chosen to be:

$$
\frac{\partial}{\partial t}\left\{\bar{P}_{i}\left(\kappa, \omega, \alpha_{1}, \ldots, \alpha_{m}\right)\right\}+\frac{\partial}{\partial x}\left\{\bar{Q}_{i}\left(\kappa, \omega, \alpha_{1}, \ldots, \alpha_{m}\right)\right\}=0, \quad i=1,2,3, \ldots, m+2 .
$$


One of these relations leads to the dispersion relation (3) whereas another yields the equation

$$
\frac{\partial \kappa}{\partial t}+\frac{\partial \omega}{\partial x}=0
$$

expressing the 'conservation of wavecrests'. (See also LightHILL [2]).

In a subsequent paper Wнітнам [10] gives a very elegant method in terms of an averaged Langrangian density function. For many conservative problems a Lagrangian density function $L\left(\eta, \eta_{x}, \eta_{t}, x, t\right)$ is explicitly known. The variational principle for $L$ yields the governing equation of the problem as Euler-Lagrange equation. Whiтнам calculates the Lagrangian density for the uniform wavetrain solution (2) and then it is averaged over one period to give the averaged Lagrangian density function $\mathscr{L}$ depending on the $m+2$ parameters $\kappa, \omega, \alpha_{1}, \alpha_{2}, \ldots, \alpha_{m}$.

For a slowly varying wavetrain it is assumed that the equation of conservation of wavecrests is valid and hence a phase function $S(x, t)$ may be introduced as follows:

$$
\kappa(x, t)=\frac{\partial S}{\partial x}, \quad \omega(x, t)=-\frac{\partial S}{\partial t} .
$$

Furthermore the parameters $\alpha_{1}, \alpha_{2}, \ldots, \alpha_{m}$ are considered as slowly varying functions of $x$ and $t$ too and for slowly varying wavetrains an averaged Lagrangian principle is assumed to hold:

$$
\delta \iint \mathscr{L}\left[S_{x}, S_{t}, \alpha_{1}(x, t), \ldots, \alpha_{m}(x, t)\right] d x d t=0 .
$$

The $m+1$ Euler-Lagrange equations

$$
\begin{aligned}
& \frac{\partial}{\partial t}\left(\frac{\partial \mathscr{L}}{\partial \omega}\right)-\frac{\partial}{\partial x}\left(\frac{\partial \mathscr{L}}{\partial \kappa}\right)=0, \\
& \frac{\partial \mathscr{L}}{\partial \alpha_{i}}=0, \quad i=1,2,3, \ldots, m,
\end{aligned}
$$

together with equation (7) provide $m+2$ equations for the $m+2$ unknown slowly varying parameters. Dispersion relation (3) is equivalent to one of the Euler-Lagrange equations (10).

For wave problems given in terms of potential functions the uniform wavetrain solution may contain a term linear in $x$ and $t$, for instance $\alpha_{1} x+\alpha_{2} t$. Then for slowly varying wavetrains the parameters $\alpha_{1}(x, t)$ and $\alpha_{2}(x, t)$, called pseudo-frequencies by WHITHAM, are related by 


$$
\frac{\partial \alpha_{1}}{\partial t}-\frac{\partial \alpha_{2}}{\partial x}=0
$$

This leads to an Euler-Lagrange equation analogous to equation (9). For further details we refer to Whitham $[10,11,12]$. An attempt to give a systematic derivation of Whitham's averaged Lagrangian principle was made by LUKE [15] who considered a non-linear Klein-Gordon equation.

LightHILL $[13,14]$ considers the restricted case of an averaged Lagrangian density $\mathscr{L}$ depending on the frequency $\omega$ and the wavenumber $\kappa$ only. The variational principle for $\mathscr{L}$ then leads to the single Euler-Lagrange equation (9). In terms of the phase function $S(x, t)$ equation (9) may be written as

$$
\frac{\partial^{2} \mathscr{L}}{\partial \omega^{2}} \frac{\partial^{2} S}{\partial t^{2}}-2 \frac{\partial^{2} \mathscr{L}}{\partial \omega \partial \kappa} \frac{\partial^{2} S}{\partial x \partial t}+\frac{\partial^{2} \mathscr{L}}{\partial \kappa^{2}} \frac{\partial^{2} S}{\partial x^{2}}=0
$$

which is a second order quasi-linear partial differential equation for $S(x, t)$. Nonlinear problems usually lead to higher order equations for $S(x, t)$ whereas linear problems yield first order equations.

\section{A general asymptotic theory of non-linear slowly varying wavetrains}

The problem is to find asymptotic solutions of non-linear dispersive wave problems representing slowly varying wavetrains, i.e. wavetrains which may be considered as nearly uniform in regions of order of magnitude of a small number of wavelengths and periods. The order of magnitude of the slow variations of wavelength, frequency, amplitude, etc. is $K^{-1}$ where $K$ is a large number. In this section we restrict ourselves again to problems described in terms of a single one-dimensional wavefunction $\eta(x, t)$.

The problem is formulated in coordinates in which wavelength and period are quantities of order unity. These coordinates are stretched with the large factor $K$ in order to obtain a set of $x, t$-coordinates in which each unity of $x$ and $t$ contains a large number (of order of magnitude $K$ ) of wavelengths and periods respectively.

In terms of these stretched coordinates $x$ and $t$ the lines of equal phase or wavefronts $S(x, t)=$ constant are defined as curves along which the normal derivative of the wavefunction $\eta(x, t)$ is of order of magnitude $K$ whereas the tangential derivative is of order unity. Accordingly it is assumed that the wavefunction of a slowly varying wavetrain may be represented by the following asymptotic series in $K$ :

$$
\eta(x, t)=U_{1}[K S(x, t), x, t]+\frac{1}{K} U_{2}[K S(x, t), x, t]+O\left(\frac{1}{K^{2}}\right) .
$$


The derivative of $\eta(x, t)$ normal to a line $S(x, t)=$ constant is given by

$$
\begin{aligned}
\frac{\partial \eta}{\partial n} & =|\nabla S|^{-1} \cdot S_{x}\left[K S_{x} U_{1 p}+U_{1 x}+S_{x} U_{2 p}+O\left(\frac{1}{K}\right)\right]+ \\
& +|\nabla S|^{-1} \cdot S_{t}\left[K S_{t} U_{1 p}+U_{1 t}+S_{t} U_{2 p}+O\left(\frac{1}{K}\right)\right]= \\
& =O(K),
\end{aligned}
$$

where $p$ stands for $K S(x, t)$. Further the tangential derivative of $\eta(x, t)$ to a wavefront $S(x, t)=$ constant is given by

$$
\begin{aligned}
\frac{\partial \eta}{\partial s} & =|\nabla S|^{-1} \cdot\left(-S_{t}\right)\left[K S_{x} U_{1 p}+U_{1 x}+S_{x} U_{2 p}+O\left(\frac{1}{K}\right)\right]+ \\
& +|\nabla S|^{-1} \cdot S_{x}\left[K S_{t} U_{1 p}+U_{1 t}+S_{t} U_{2 p}+O\left(\frac{1}{K}\right)\right]= \\
& =O(1)
\end{aligned}
$$

In regions of order of magnitude $K^{-1}$ in the $x$, $t$-plane the variations of $p=K S(x, t)$ are of order unity. Thus the dependence of $U_{i}$ on $p$ describes the local rapid oscillations (it is anticipated that the dependence of $U_{i}$ on $p$ may be oscillatory) and the slow dependence on $x$ and $t$ describes the large-scale variations of the wavetrain.

In the subsequent chapters of this thesis we will insert asymptotic expansion (12) for slowly varying wavetrains (or an appropriate modification of it in order to deal with more than one wavefunction or with more than one dimension) into the basic equations of three non-linear dispersive water wave problems. By equating to zero the coefficients of the various powers of $K$ we obtain differential equations for $U_{1}(p, x, t)$ and $U_{2}(p, x, t)$. It appears that the coefficient of the highest power of $K$ leads to an ordinary differential equation for the dependence of the leading term $U_{1}(p, x, t)$ on $p$. This equation is identical with the ordinary differential equation for the uniform wavetrain.

The slow dependence of the leading term on $x$ and $t$ is governed by the slowly varying parameters $\kappa(x, t), \omega(x, t), \alpha_{1}(x, t), \alpha_{2}(x, t), \ldots, \alpha_{m}(x, t)$. These parameters satisfy dispersion relation (3) and the equation $\kappa_{t}+\omega_{x}=0$. The remaining equations are obtained by imposing conditions of boundedness on the second term $U_{2}(p, x, t)$ of the asymptotic series which is determined by a linear differential equation in $p$ with an inhomogeneous periodic righthand side depending on $U_{1}(p, x, t)$. In order to ensure boundedness of $U_{2}(p, x, t)$ as a function of $p$ several integral relations must be satisfied by $U_{1}(p, x, t)$. The integral relations provide the remaining equations for the determination of the slowly varying parameters of the problem. For the shallow 
water waves of Chapters I and II it is shown that these integral relations also may be obtained by applying an appropriate averaging technique to conservative equations of the problem. This averaging technique is different from Whitham's averaging of conservation laws.

In order to render the equations for the slowly varying parameters tractable the leading term $U_{1}(p, x, t)$ is developed in an asymptotic series in powers of the small amplitude/depth ratio $\varepsilon$ and only the lowest order non-linear effects are taken into account. This leads to a set of partial differential equations for wavenumber, frequency, amplitude, mean waveheight, etc.

In Chapters I and II these equations are investigated further by a transformation into their characteristic form. It appears that two equations for wavenumber and amplitude uncouple from the other ones. These equations may be transformed into the equations of one-dimensional unsteady gas dynamics and an asymptotic solution of the initial value problem for slowly varying wavetrains is given. Chapter III deals with Stokes waves and provides a systematic derivation of the results found by Wнітнам $[11,12]$ with the averaged Lagrangian density method. In the Appendix the uniform progressive periodic Stokes wave is derived and expanded in powers of the small amplitude/wavelength ratio $\varepsilon$. 


\section{Chapter I}

\section{CNOIDAL WAVES}

\section{I.1 The Korteweg-de Vries equation}

Cnoidal waves are solutions of a differential equation derived by KoRTEwEG and DE VRIES [16] for one-dimensional shallow water waves. This equation may be written as follows:

$$
\frac{\partial \bar{\eta}}{\partial \bar{t}}+\sqrt{g h_{0}}\left(1+\frac{3 \bar{\eta}}{2 h_{0}}\right) \frac{\partial \bar{\eta}}{\partial \bar{x}}+\frac{1}{6} h_{0}^{2} \sqrt{g h_{0}} \frac{\partial^{3} \bar{\eta}}{\partial \bar{x}^{3}}=0,
$$

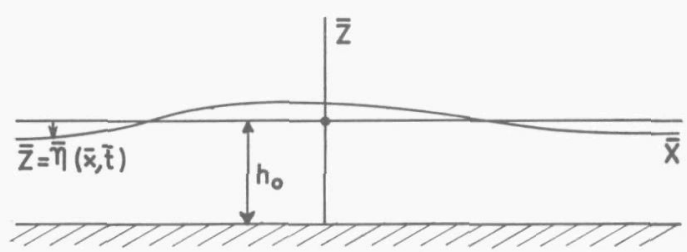

Fig. 1.

where $\bar{\eta}(\bar{x}, \bar{t})$ denotes the elevation of the free surface above the uniform undisturbed depth $h_{0}$. The equation is valid for small values of the two non-dimensional parameters $\varepsilon$ and $\mu$ defined by

$$
\varepsilon=\frac{a}{h_{0}}, \quad \mu=\frac{h_{0}^{2}}{6 \lambda_{0}^{2}},
$$

where $a$ is a typical amplitude and $\lambda_{0}$ a typical wavelength. Introducing non-dimensional variables $\eta, x$ and $t$ by means of

$$
\varepsilon \eta=\frac{3 \bar{\eta}}{2 h_{0}}, \quad x=\frac{\bar{x}}{\lambda_{0}}, \quad t=\frac{\sqrt{g h_{0}}}{\lambda_{0}} \bar{t},
$$

equation (1.1) transforms into:

$$
\frac{\partial \eta}{\partial t}+(1+\varepsilon \eta) \frac{\partial \eta}{\partial x}+\mu \frac{\partial^{3} \eta}{\partial x^{3}}=0 .
$$

In this non-dimensional equation the waveheight $\eta(x, t)$, the wavelength and period are all quantities of order of magnitude one. 
It is worthwhile to give some attention to the linearized versions of equation (1.2). If both $\varepsilon$ and $\mu$ approach zero we get a unidirectional wave equation with general solution

$$
\eta(x, t)=F(x-t) .
$$

This means that each disturbance of the free surface is propagated without distortion with non-dimensional velocity one in the direction of the positive $x$-axis. This corresponds to the fully linearized shallow water theory in which no dispersion occurs. For our purposes the linearization with respect to $\varepsilon$ only is more meaningful because the presence of the third order derivative yields a dispersive linear equation:

$$
\frac{\partial \eta}{\partial t}+\frac{\partial \eta}{\partial x}+\mu \frac{\partial^{3} \eta}{\partial x^{3}}=0
$$

Substitution of a uniform harmonic wave

$$
\eta(x, t)=A \cos [2 \pi(\kappa x-\omega t)]
$$

gives the dispersion relation between frequency and wavenumber for equation (1.3):

$$
\omega=\omega_{0}(\kappa)=\kappa-4 \pi^{2} \mu \kappa^{3} .
$$

In the following we will always refer to equation (1.3) as to the linearized equation for cnoidal waves.

\section{I.2 Asymptotic expansion with respect to $K$}

In order to investigate asymptotic solutions of equation (1.2) representing slowly varying wavetrains the coordinates $x$ and $t$ are stretched with a large factor $K$ :

$$
x=K x^{*}, \quad t=K t^{*} .
$$

We then get after omission of the asterisks:

$$
\frac{\partial \eta}{\partial t}+(1+\varepsilon \eta) \frac{\partial \eta}{\partial x}+\frac{\mu}{K^{2}} \frac{\partial^{3} \eta}{\partial x^{3}}=0
$$

Now each unity of $x$ and $t$ contains a large number (of order $K$ ) of wavelengths and periods respectively. If we consider an initial value problem with a given slowly varying wavetrain for $t=0$, then the large parameter $K$ is determined by the largescale variations of the wavetrain at $t=0$. 
Substitution of the general asymptotic expansion for a slowly varying wavetrain:

$$
\eta(x, t)=U[K S(x, t), x, t]+\frac{1}{K} V[K S(x, t), x, t]+O\left(\frac{1}{K^{2}}\right)
$$

into equation (1.5) yields with the abbreviation $p=K S(x, t)$ :

$$
\begin{aligned}
& K S_{t} U_{p}+U_{t}+V_{p} S_{t}+\left(1+\varepsilon U+\frac{\varepsilon}{K} V\right)\left(K S_{x} U_{p}+U_{x}+V_{p} S_{x}\right)+ \\
& +\frac{\mu}{K^{2}}\left[K^{3} S_{x}^{3} U_{p p p}+K^{2}\left(3 S_{x}^{2} U_{p p x}+3 S_{x} S_{x x} U_{p p}+S_{x}^{3} V_{p p p}\right)\right]+O\left(\frac{1}{K}\right)=0 .
\end{aligned}
$$

Introducing the local wavenumber $\kappa$ and the local frequency $\omega$ by

$$
\kappa=S_{x}, \quad \omega=-S_{t},
$$

and equating to zero the coefficients of the various powers of $K$ in equation (1.6), we get successively:

$$
\begin{array}{ll}
O(K): \quad(\kappa-\omega) U_{p}+\varepsilon \kappa U U_{p}+\mu \kappa^{3} U_{p p p}=0, \\
O(1): \quad(\kappa-\omega) V_{p}+\varepsilon \kappa\left(U V_{p}+V U_{p}\right)+\mu \kappa^{3} V_{p p p}=F(p, x, t),
\end{array}
$$

with the notation:

$$
F(p, x, t)=-U_{t}-U_{x}-\varepsilon U U_{x}-3 \mu\left(\kappa^{2} U_{p p x}+\kappa \kappa_{x} U_{p p}\right) .
$$

Equation (1.7) may be considered as an ordinary differential equation for $U$ as a function of $p$ with coefficients depending on $x$ and $t$. Integrating equation (1.7) twice with respect to $p$ we find successively:

$$
\begin{aligned}
& (\kappa-\omega) U+\frac{1}{2} \varepsilon \kappa U^{2}+\mu \kappa^{3} U_{p p}=\frac{1}{2} \alpha, \\
& \mu \kappa^{3} U_{p}^{2}=\alpha U+\beta+(\omega-\kappa) U^{2}-\frac{1}{3} \varepsilon \kappa U^{3},
\end{aligned}
$$

with $\alpha$ and $\beta$ unknown functions of $x$ and $t$ figuring as constants of integration. From equation (1.9) it can be seen that $U$ is a periodic function of $p$ oscillating between two zeroes $U_{1}$ and $U_{2}$ of the righthand side of equation (1.9). In general this righthand side has three zeroes but we have to select those two zeroes $U_{1}$ and $U_{2}$ for which the righthand side of equation (1.9) is positive for $U_{1}<U<U_{2}$. 
The dependence of $U$ on $p$ can be given in implicit form as:

$$
p+\gamma=\sqrt{\mu \kappa^{3}} \int_{U_{1}}^{U} \frac{d U}{\left[\alpha U+\beta+(\omega-\kappa) U^{2}-\frac{1}{3} \varepsilon \kappa U^{3}\right]^{\frac{1}{2}}},
$$

with $\gamma(x, t)$ as a non-essential shifting constant. The explicit dependence of $U$ on $p$ is in terms of the Jacobian elliptic function $\mathrm{cn}$, the fact from which the cnoidal waves derive their name.

The period $p_{0}$ of $U$ should be independent of $x$ and $t$, otherwise differentiation of the relation

$$
U\left(p+n p_{0}, x, t\right)=U(p, x, t), \quad(n \text { integer })
$$

with respect to $x$ or $t$ would give rise to unbounded terms for large $n$ :

$$
U_{x}\left(p+n p_{0}, x, t\right)=U_{x}(p, x, t)-n p_{0 x} U_{p}(p, x, t) .
$$

Hence $p_{0}$ is a constant and may be normalized to unity, which gives the dispersion relation:

$$
\frac{1}{2}=\sqrt{\mu \kappa^{3}} \int_{U_{1}}^{U_{2}} \frac{d U}{\left[\alpha U+\beta+(\omega-\kappa) U^{2}-\frac{1}{3} \varepsilon \kappa U^{3}\right]^{\frac{1}{2}}} .
$$

Because of the dependence of $U_{1}$ and $U_{2}$ on $\omega, \kappa, \alpha$ and $\beta$ relation (1.10) is a transcendental relation between $\omega, \kappa, \alpha$ and $\beta$. For $\varepsilon=0$ we have $\alpha=0$ and $\beta=\kappa-\omega$. Then equation (1.10) becomes:

$$
\frac{1}{2}=\sqrt{\frac{\mu \kappa^{3}}{\kappa-\omega}} \int_{-1}^{+1} \frac{d U}{\sqrt{1-U^{2}}}=\pi \sqrt{\frac{\mu \kappa^{3}}{\kappa-\omega}},
$$

which is equivalent to dispersion relation (1.4) for the linearized problem.

Hence we see that the first term $U(p, x, t)$ of the asymptotic expansion for the slowly varying wavetrain represents locally a uniform wavetrain as a result of the periodic dependence of $U$ on $p$. The slow variations of the wavetrain are described by the four slowly varying parameters $\kappa(x, t), \omega(x, t), \alpha(x, t)$ and $\beta(x, t)$ which occur in $U(p, x, t)$, and we are interested in finding four equations to determine these functions. Dispersion relation (1.10) and the relation

$$
\frac{\partial \omega}{\partial x}+\frac{\partial \kappa}{\partial t}=0
$$


which follows from the definition of $\kappa$ and $\omega$, provide two of them. The two remaining equations will be obtained in the next section from the condition of boundedness of the second term $V(p, x, t)$ in the asymptotic expansion for the wavefunction $\eta(x, t)$.

\section{I.3 Two integral relations for $U(p, x, t)$}

The second term $V(p, x, t)$ in the asymptotic series for the slowly varying wavetrain is determined from equation (1.8). This equation may be written as:

$$
\frac{\partial}{\partial p}\left[(\kappa-\omega) V+\varepsilon \kappa U V+\mu \kappa^{3} V_{p p}\right]=F(p, x, t)
$$

The term between square brackets has to be bounded, so:

$$
\int F(p, x, t) d p+p_{1}(x, t)
$$

must be bounded, where $p_{1}(x, t)$ is a constant of integration. Because $F(p, x, t)$ is periodic in $p$ with period 1 , this integral is bounded for large $p$ only if the integral over one period vanishes:

$$
\int_{0}^{1} F(p, x, t) d p=0
$$

or, written out fully:

$$
\begin{aligned}
& \int_{0}^{1}\left[U_{t}+U_{x}+\varepsilon U U_{x}+3 \mu\left(\kappa^{2} U_{p p x}+\kappa \kappa_{x} U_{p p}\right)\right] d p= \\
& =\int_{0}^{1}\left[U_{t}+U_{x}+\varepsilon U U_{x}\right] d p=0,
\end{aligned}
$$

where use has been made of the periodicity of $U_{p x}$ and $U_{p}$.

Equation (1.14) is the third relation between $\kappa(x, t), \omega(x, t), \alpha(x, t)$ and $\beta(x, t)$. A fourth relation follows after integration of equation (1.12) with respect to $p$ and noticing that $V=U_{p}$ is a solution of the corresponding homogeneous equation. Introducing a function $w$ by putting:

$$
V=w U_{p},
$$

we find after some manipulation:

$$
\mu \kappa^{3} \frac{\partial}{\partial p}\left(w_{p} U_{p}^{2}\right)=\mu \kappa^{3} \frac{\partial}{\partial p}\left[V_{p} U_{p}-V U_{p p}\right]=U_{p}\left\{\int F(p, x, t) d p+p_{1}(x, t)\right\} .
$$


With the same reasoning as above the term between square brackets must be bounded for large $p$ and because of the periodicity of the inhomogeneous term, the integral over one period of it must vanish:

$$
\int_{0}^{1} U_{p}\left\{\int^{p} F(\tilde{p}, x, t) d \tilde{p}+p_{1}(x, t)\right\} d p=0,
$$

which is transformed by partial integration into:

$$
\left[U \int F(p, x, t) d p+U p_{1}\right]_{p=0}^{1}-\int_{0}^{1} U F d p=-\int_{0}^{1} U F d p=0,
$$

or written out fully:

$$
\int_{0}^{1} U\left[U_{t}+U_{x}+\varepsilon U U_{x}+3 \mu\left(\kappa^{2} U_{p p x}+\kappa \kappa_{x} U_{p p}\right)\right] d p=0 .
$$

The four equations (1.10), (1.11), (1.14) and (1.15) determine the four slowly varying functions $\kappa(x, t), \omega(x, t), \alpha(x, t)$ and $\beta(x, t)$. In the next section we will show that integral relations (1.14) and (1.15) also may be obtained by an appropriate averaging procedure applied to conservation equations for cnoidal waves.

\section{I.4 Conservation equations for cnoidal waves}

Several conservation laws can be obtained from the governing equation (1.5). Equation (1.5) itself is an equation in conservative form:

$$
\frac{\partial}{\partial t}(\eta)+\frac{\partial}{\partial x}\left(\eta+\frac{1}{2} \varepsilon \eta^{2}+\frac{\mu}{K^{2}} \eta_{x x}\right)=0 .
$$

A second conservation law may be constructed by multiplication with $\eta$ :

$$
\begin{aligned}
& \eta \frac{\partial}{\partial t}(\eta)+\eta \frac{\partial}{\partial x}\left(\eta+\frac{1}{2} \varepsilon \eta^{2}+\frac{\mu}{K^{2}} \eta_{x x}\right)= \\
& =\frac{\partial}{\partial t}\left(\frac{1}{2} \eta^{2}\right)+\frac{\partial}{\partial x}\left\{\frac{1}{2} \eta^{2}+\frac{1}{3} \varepsilon \eta^{3}+\frac{\mu}{K^{2}}\left(\eta \eta_{x x}-\frac{1}{2} \eta_{x}^{2}\right)\right\}=0 .
\end{aligned}
$$

Higher order conservation equations are obtained by multiplication with $\eta^{2}, \eta^{3}$, etc., but these will not be needed here. Equation (1.16) may be considered as an approximate form of the equation of conservation of mass and equation (1.17) as the corresponding approximation to the equation of conservation of energy. 
Consider the uniform wavetrain:

$$
\eta(x, t)=U[K(\kappa x-\omega t) ; \kappa, \omega, \alpha, \beta]
$$

satisfying equation (1.7) with $p=K(\kappa x-\omega t)$ :

$$
(\kappa-\omega) U_{p}+\varepsilon \kappa U U_{p}+\mu \kappa^{3} U_{p p p}=0,
$$

with parameters $\kappa, \omega, \alpha$ and $\beta$ satisfying dispersion relation (1.10). It is assumed that a slowly varying wavetrain can be represented locally, i.e. in regions of order $K^{-1}$ in the $x, t$-plane, by the uniform wavetrain solution (1.18) of equation (1.19), but with slowly varying parameters $\kappa(x, t), \omega(x, t), \alpha(x, t)$ and $\beta(x, t)$, which still satisfy dispersion relation (1.10).

Hence we put for a slowly varying wavetrain:

with

$$
\eta(x, t)=U[p ; \kappa(x, t), \omega(x, t), \alpha(x, t), \beta(x, t)],
$$

$$
p_{x}=K \kappa(x, t), \quad p_{t}=-K \omega(x, t) .
$$

Introducing the notation

$$
U_{x}=U_{\alpha} \alpha_{x}+U_{\beta} \beta_{x}+U_{\kappa} \kappa_{x}+U_{\omega} \omega_{x},
$$

and similarly for $U_{t}$, we get after substitution of equation (1.20) into conservation equations (1.16) and (1.17):

$$
\begin{aligned}
& -K \omega U_{p}+U_{t}+(1+\varepsilon U)\left(K \kappa U_{p}+U_{x}\right)+ \\
& +\frac{\mu}{K^{2}}\left[K^{3} \kappa^{3} U_{p p p}+K^{2}\left(3 \kappa^{2} U_{p p x}+3 \kappa \kappa_{x} U_{p p}\right)\right]+O\left(\frac{1}{K}\right)=0,
\end{aligned}
$$

and

$$
\begin{aligned}
& U\left[-K \omega U_{p}+U_{t}+(1+\varepsilon U)\left(K \kappa U_{p}+U_{x}\right)+\right. \\
& \left.+\frac{\mu}{K^{2}}\left\{K^{3} \kappa^{3} U_{p p p}+K^{2}\left(3 \kappa^{2} U_{p p x}+3 \kappa \kappa_{x} U_{p p}\right)\right\}\right]+O\left(\frac{1}{K}\right)=0 .
\end{aligned}
$$

By virtue of equation (1.19) the terms of order $K$ in equations (1.21) and (1.22) vanish and the remaining parts, being of order unity, are averaged over one period in $p$ in order to obtain equations for the $x, t$-dependence of the wavetrain:

$$
\begin{aligned}
& \int_{0}^{1}\left[U_{t}+U_{x}+\varepsilon U U_{x}+3 \mu\left(\kappa^{2} U_{p p x}+\kappa \kappa_{x} U_{p p}\right)\right] d p+O\left(\frac{1}{K}\right)= \\
& =\int_{0}^{1} F(p, x, t) d p+O\left(\frac{1}{K}\right)=0
\end{aligned}
$$




$$
\begin{aligned}
& \int_{0}^{1} U\left[U_{t}+U_{x}+\varepsilon U U_{x}+3 \mu\left(\kappa^{2} U_{p p x}+\kappa \kappa_{x} U_{p p}\right)\right] d p+O\left(\frac{1}{K}\right)= \\
& =\int_{0}^{1} U(p, x, t) F(p, x, t) d p+O\left(\frac{1}{K}\right)=0 .
\end{aligned}
$$

These two relations are, apart from terms of order $K^{-1}$ which may be omitted to the present order of approximation, in exact agreement with integral relations (1.14) and (1.15) of the last section.

\section{I.5 Asymptotic expansion of $U(p, x, t)$ in powers of $\varepsilon$}

The system of four equations (1.10), (1.11), (1.14), and (1.15) for the determination of $\kappa(x, t), \omega(x, t), \alpha(x, t)$ and $\beta(x, t)$ is very complicated and cannot be dealt with in its present form. An asymptotic expansion of $U(p, x, t)$ with respect to the small amplitude/depth ratio $\varepsilon$ enables us to simplify the system considerably. This asymptotic expansion of $U(p, x, t)$ which will appear to be of the form of a Fourier series in $p$ with coefficients depending on $x, t$ and $\varepsilon$, is inserted into the equations for $\kappa, \omega$, $\alpha$ and $\beta$, taking into account only the lowest order non-linear effects. This means that we consider the first non-linear approximation beyond the linear infinitesimal amplitude approximation.

Furthermore the functions $\alpha(x, t)$ and $\beta(x, t)$ have no physical significance; we want to obtain equations in terms of wavenumber, frequency, amplitude and mean waveheight. To this end new functions $\eta_{0}(x, t), A(x, t)$ and $\Phi(p, x, t)$ are introduced by means of

$$
\begin{aligned}
& U(p, x, t)=\eta_{0}(x, t)+A(x, t) \Phi(p, x, t), \\
& \eta_{0}(x, t)=\frac{1}{2}\left\{U_{2}(x, t)+U_{1}(x, t)\right\} .
\end{aligned}
$$

Then $A(x, t)$ has the meaning of an amplitude function and $\Phi(p, x, t)$ is a periodic function of $p$ with period one. The introduction of $\eta_{0}(x, t)$ allows us to fix the extremal values of $\Phi(p, x, t)$ as -1 and +1 . Note that $\eta_{0}(x, t)$ is not equal to the mean waveheight, i.e. the averaged value of $U(p, x, t)$ over one period in $p$. For computational reasons the function $\eta_{0}(x, t)$ has advantages and only in a later stage of the analysis we will switch to the mean waveheight.

It is possible now to eliminate the functions $\alpha(x, t)$ and $\beta(x, t)$ and have them replaced by $A(x, t)$ and $\eta_{0}(x, t)$. Substituting (1.23) into equation (1.9) we obtain a differential equation of $\Phi(p, x, t)$ :

$$
\mu \kappa^{3} A^{2} \Phi_{p}^{2}=\alpha\left(\eta_{0}+A \Phi\right)+\beta+(\omega-\kappa)\left(\eta_{0}+A \Phi\right)^{2}-\frac{1}{3} \varepsilon \kappa\left(\eta_{0}+A \Phi\right)^{3} .
$$


Using the fact that $\Phi_{p}=0$ for both $\Phi=-1$ and $\Phi=+1$, we arrive at two algebraical equations from which $\alpha$ and $\beta$ may be expressed as functions of $\omega, \kappa, A$ and $\eta_{0}$ :

$$
\begin{aligned}
& \alpha\left(\eta_{0}+A\right)+\beta+(\omega-\kappa)\left(\eta_{0}+A\right)^{2}-\frac{1}{3} \varepsilon \kappa\left(\eta_{0}+A\right)^{3}=0, \\
& \alpha\left(\eta_{0}-A\right)+\beta+(\omega-\kappa)\left(\eta_{0}-A\right)^{2}-\frac{1}{3} \varepsilon \kappa\left(\eta_{0}-A\right)^{3}=0 .
\end{aligned}
$$

Solving $\alpha$ and $\beta$ and inserting the result into equation (1.24), we arrive at the following differential equation for $\Phi(p, x, t)$ :

$$
\mu \kappa^{3} \Phi_{p}^{2}=\left(1-\Phi^{2}\right)\left(\frac{1}{3} \varepsilon A \kappa \Phi+\varepsilon \kappa \eta_{0}+\kappa-\omega\right) .
$$

Before proceeding we fix the order of magnitude of $A(x, t)$ and $\eta_{0}(x, t)$. It is clear that $A(x, t)$ is of order unity because $\eta(x, t)$ is of order unity. For $\varepsilon \rightarrow 0$ we get the linear wave problem for which $\eta_{0}(x, t)$ is trivial: the surface elevation in the linear case may be taken symmetrical with respect to the undisturbed state. For $\varepsilon \neq 0$ it is assumed that $\eta_{0}(x, t)$ is of order $\varepsilon$ and hence we put:

$$
\eta_{0}(x, t)=\varepsilon \eta_{1}(x, t), \quad \eta_{1}(x, t)=O(1) .
$$

Then equation (1.25) becomes:

$$
\mu \kappa^{3} \Phi_{p}^{2}=\left(1-\Phi^{2}\right)\left(\frac{1}{3} \varepsilon A \kappa \Phi+\varepsilon^{2} \kappa \eta_{1}+\kappa-\omega\right) .
$$

The condition that $\Phi(p, x, t)$ has period one in $p$ yields the dispersion relation

$$
\frac{1}{2}=\sqrt{\mu \kappa^{3}} \int_{-1}^{+1} \frac{d \Phi}{\left[\left(1-\Phi^{2}\right)\left(\frac{1}{3} \varepsilon A \kappa \Phi+\varepsilon^{2} \kappa \eta_{1}+\kappa-\omega\right)\right]^{\frac{1}{2}}} .
$$

For $\varepsilon=0$ equation (1.26) has the linearized solution:

$$
\Phi(p, x, t)=\cos \left\{p \sqrt{\frac{\kappa-\omega}{\mu \kappa^{3}}}\right\},
$$

and we apply Lindstedt's method (MINORSKY [17]), in order to obtain an asymptotic expansion of the solution of equation (1.26) in the form of a Fourier series in $p$ starting with $(1.28)$ as the first harmonic term.

Differentiation of equation (1.26) with respect to $p$ and introduction of the new independent variable

$$
q=p \sqrt{\frac{\kappa-\omega}{\mu \kappa^{3}}},
$$


yields the equation

$$
(\kappa-\omega)\left(\Phi_{q q}+\Phi\right)=-\frac{1}{2} \varepsilon A \kappa \Phi^{2}-\varepsilon^{2} \eta_{1} \kappa \Phi+\frac{1}{6} \varepsilon A \kappa .
$$

It is essential for Lindstedt's method that both the dependent variable $\Phi$ and the independent variable $q$ are considered as functions of a new variable $s$ and both are expanded in a power series in $\varepsilon$ :

$$
\begin{aligned}
\Phi(s) & =\cos s+\varepsilon \Phi_{1}(s)+\varepsilon^{2} \Phi_{2}(s)+\varepsilon^{3} \Phi_{3}(s)+\ldots, \\
q & =s\left(1+c_{1} \varepsilon+c_{2} \varepsilon^{2}+c_{3} \varepsilon^{3}+\ldots\right) .
\end{aligned}
$$

The introduction of the constants $c_{1}, c_{2}, c_{3}, \ldots$ enables us to avoid secular terms, i.e. terms that are not periodic in $s$. The boundary conditions are:

$$
\begin{aligned}
& \Phi_{1}(0)=\Phi_{2}(0)=\Phi_{3}(0)=\ldots=0, \\
& \Phi_{1}^{\prime}(0)=\Phi_{2}^{\prime}(0)=\Phi_{3}^{\prime}(0)=\ldots=0 .
\end{aligned}
$$

Substitution of equations (1.30) and (1.31) into equation (1.29) gives

$$
\begin{aligned}
& -\cos s+\varepsilon \Phi_{1}^{\prime \prime}+\varepsilon^{2} \Phi_{2}^{\prime \prime}+\ldots+\left[1+2 \varepsilon c_{1}+\varepsilon^{2}\left(c_{1}^{2}+2 c_{2}\right)+\ldots\right] \times \\
& \times\left[\cos s+\varepsilon \Phi_{1}+\varepsilon^{2} \Phi_{2}+\ldots\right]=\left[1+2 \varepsilon c_{1}+\varepsilon^{2}\left(c_{1}^{2}+2 c_{2}\right)+\ldots\right] \times \\
& \times\left[\frac{A \kappa \varepsilon}{6(\kappa-\omega)}-\frac{\varepsilon^{2} \kappa \eta_{1}}{\kappa-\omega}\left(\cos s+\varepsilon \Phi_{1}+\varepsilon^{2} \Phi_{2}+\ldots\right)+\right. \\
& \left.-\frac{\varepsilon A \kappa}{2(\kappa-\omega)}\left(\cos ^{2} s+2 \varepsilon \Phi_{1} \cos s+\ldots\right)\right] .
\end{aligned}
$$

The term of order unity vanishes and the coefficient of $\varepsilon$ yields a differential equation for $\Phi_{1}(s)$ :

$$
\frac{d^{2} \Phi_{1}}{d s^{2}}+\Phi_{1}=-2 c_{1} \cos s+\frac{A \kappa}{6(\kappa-\omega)}-\frac{A \kappa}{2(\kappa-\omega)} \cos ^{2} s .
$$

The term $-2 c_{1} \cos s$ in the righthand side gives rise to a term proportional to $s \sin s$ in $\Phi_{1}(s)$. This is a secular term destroying the periodicity of $\Phi_{1}(s)$. Hence we take $c_{1}=0$.

The solution of equation (1.33) satisfying the boundary conditions is given by:

$$
\Phi_{1}(s)=-\frac{A \kappa}{12(\kappa-\omega)}(1-\cos 2 s) .
$$


The coefficient of $\varepsilon^{2}$ in equation (1.32) produces a differential equation for $\Phi_{2}(s)$ :

$$
\frac{d^{2} \Phi_{2}}{d s^{2}}+\Phi_{2}=\left[-\frac{\kappa \eta_{1}}{\kappa-\omega}-2 c_{2}+\frac{A^{2} \kappa^{2}}{24(\kappa-\omega)^{2}}\right] \cos s-\frac{A^{2} \kappa^{2}}{24(\kappa-\omega)^{2}} \cos 3 s .
$$

Here again the term with coss gives rise to secular terms in $\Phi_{2}(s)$ and hence its coefficient must vanish. This gives for $c_{2}$ :

$$
c_{2}=\frac{A^{2} \kappa^{2}}{48(\kappa-\omega)^{2}}-\frac{\eta_{1} \kappa}{2(\kappa-\omega)} .
$$

After solving $\Phi_{2}(s)$ and proceeding with the equation for $\Phi_{3}(s)$ which yields $c_{3}=0$, we get the following asymptotic solution of equation (1.26):

$$
\begin{aligned}
\Phi(s) & =\cos s-\frac{\varepsilon A \kappa}{12(\kappa-\omega)}(1-\cos 2 s)+ \\
& +\frac{\varepsilon^{2} A^{2} \kappa^{2}}{192(\kappa-\omega)^{2}}(\cos 3 s-\cos s)+O\left(\varepsilon^{3}\right),
\end{aligned}
$$

with

$$
q=p \sqrt{\frac{\kappa-\omega}{\mu \kappa^{3}}}=s\left[1+\varepsilon^{2}\left\{\frac{A^{2} \kappa^{2}}{48(\kappa-\omega)^{2}}-\frac{\eta_{1} \kappa}{2(\kappa-\omega)}\right\}+O\left(\varepsilon^{4}\right)\right] .
$$

The condition that $\Phi(p)$ is periodic with period one gives $s=2 \pi p$ and equation (1.35) yields the dispersion relation:

$$
\kappa-\omega=4 \pi^{2} \mu \kappa^{3}\left[1+\varepsilon^{2}\left\{\frac{A^{2} \kappa^{2}}{24(\kappa-\omega)^{2}}-\frac{\eta_{1} \kappa}{\kappa-\omega}\right\}\right]+O\left(\varepsilon^{4}\right) .
$$

This relation also could have been obtained by expansion of the complete dispersion relation (1.27) in powers of $\varepsilon$. Equation (1.36) is written more conveniently as

$$
\omega=\kappa-4 \pi^{2} \mu \kappa^{3}+\varepsilon^{2}\left\{\eta_{1} \kappa-\frac{A^{2}}{96 \pi^{2} \mu \kappa}\right\}+O\left(\varepsilon^{4}\right),
$$

and similarly we can write for $U(p, x, t)$ :

$$
U=\varepsilon \eta_{1}+A \cos 2 \pi p+\frac{\varepsilon A^{2}}{48 \pi^{2} \mu \kappa^{2}}\{\cos 4 \pi p-1\}+O\left(\varepsilon^{2}\right) .
$$


Introducing the mean waveheight $\varepsilon h_{1}(x, t)$ as the averaged value of $U(p, x, t)$ over one period in $p$ :

$$
\varepsilon h_{1}(x, t)=\varepsilon \eta_{1}-\frac{\varepsilon A^{2}}{48 \pi^{2} \mu \kappa^{2}}+O\left(\varepsilon^{2}\right), \quad h_{1}=O(1),
$$

we arrive at

$$
U=\varepsilon h_{1}+A \cos 2 \pi p+\frac{\varepsilon A^{2}}{48 \pi^{2} \mu \kappa^{2}} \cos 4 \pi p+O\left(\varepsilon^{2}\right) .
$$

Dispersion relation (1.37) is transformed into:

$$
\omega=\omega_{0}(\kappa)+\varepsilon^{2}\left\{\kappa h_{1}+\frac{A^{2}}{96 \pi^{2} \mu \kappa}\right\}+O\left(\varepsilon^{4}\right)
$$

with the abbreviation

$$
\omega_{0}(\kappa)=\kappa-4 \pi^{2} \mu \kappa^{3} .
$$

Notice that for $\varepsilon=0$ dispersion relation (1.39) reduces to dispersion relation (1.4) of the linear problem and hence the dependence of $\omega$ on $A$ and $h_{1}$ is a non-linear effect. Equation (1.38) is the final form of the asymptotic expansion of $U(p, x, t)$ with respect to $\varepsilon$ and is used for substitution into integral relations (1.14) and (1.15). Writing relation (1.14) as:

$$
\frac{\partial}{\partial t} \int_{0}^{1} U d p+\frac{\partial}{\partial x} \int_{0}^{1} U d p+\frac{1}{2} \varepsilon \frac{\partial}{\partial x} \int_{0}^{1} U^{2} d p=0,
$$

and integral relation (1.15) as:

$$
\begin{aligned}
& \frac{1}{2} \frac{\partial}{\partial x} \int_{0}^{1} U^{2} d p+\frac{1}{2} \frac{\partial}{\partial t} \int_{0}^{1} U^{2} d p+\frac{1}{3} \varepsilon \frac{\partial}{\partial x} \int_{0}^{1} U^{3} d p+ \\
& +3 \mu \kappa^{2} \int_{0}^{1} U U_{p p x} d p+3 \mu \kappa \kappa_{x} \int_{0}^{1} U U_{p p} d p=0
\end{aligned}
$$

insertion of equation (1.38) gives successively:

$$
\begin{aligned}
& \frac{\partial h_{1}}{\partial t}+\frac{\partial h_{1}}{\partial x}+\frac{\partial}{\partial x}\left(\frac{1}{4} A^{2}\right)+O\left(\varepsilon^{2}\right)=0 \\
& \frac{\partial}{\partial t}\left(A^{2}\right)+\frac{\partial}{\partial x}\left\{\left(1-12 \pi^{2} \mu \kappa^{2}\right) A^{2}\right\}+O\left(\varepsilon^{2}\right)=0 .
\end{aligned}
$$


Introducing the second order functions $H_{0}(x, t)$ and $E(x, t)$ by means of

$$
H_{0}=\varepsilon^{2} h_{1}, \quad E=\varepsilon^{2} A^{2},
$$

equations (1.40) and (1.41) may be written apart from terms of order $\varepsilon^{4}$, as:

$$
\begin{gathered}
\frac{\partial H_{0}}{\partial t}+\frac{\partial}{\partial x}\left\{H_{0}+\frac{1}{4} E\right\}=0, \\
\frac{\partial E}{\partial t}+\frac{\partial}{\partial x}\left\{\omega_{0}^{\prime}(\kappa) E\right\}=0 .
\end{gathered}
$$

Dispersion relation (1.39) is written as

$$
\omega=\omega_{0}(\kappa)+\kappa H_{0}+\frac{E}{96 \pi^{2} \mu \kappa}+O\left(\varepsilon^{4}\right)
$$

Equation (1.42) may be considered as an averaged form of the equation of conservation of mass and equation (1.43) expresses the conservation of the 'averaged energy' $E(x, t)$ of the wavetrain, which is propagated apparently with linear group velocity

$$
C_{0}=\frac{d \omega_{0}}{d \kappa}=1-12 \pi^{2} \mu \kappa^{2}
$$

within the present order of approximation.

In the next section these equations are investigated further.

\section{I.6 The equations for $\kappa, \omega, E$ and $H_{0}$}

The set of equations (1.11), (1.42), (1.43) and (1.44) determining the four functions $\kappa(x, t), \omega(x, t), E(x, t)$ and $H_{0}(x, t)$ is reduced to a set of three equations by elimination of $\omega(x, t)$. Differentiation of dispersion relation (1.44) with respect to $x$ and using equation (1.11) gives the equation:

$$
\frac{\partial \kappa}{\partial t}+\omega_{0}^{\prime}(\kappa) \frac{\partial \kappa}{\partial x}+H_{0} \frac{\partial \kappa}{\partial x}+\kappa \frac{\partial H_{0}}{\partial x}+\frac{\kappa \frac{\partial E}{\partial x}-E \frac{\partial \kappa}{\partial x}}{96 \pi^{2} \mu \kappa^{2}}=0 .
$$

Much information about the set of equations (1.42), (1.43) and (1.45) may be obtained from an investigation of the characteristic velocities. These characteristic velocities are found by multiplying equations (1.42) and (1.43) with factors $\lambda$ and $v$ respectively 
and adding them to equation (1.45). The condition that $\kappa, E$ and $H_{0}$ are to be differentiated in the same characteristic direction $C$ gives a set of algebraical equations for $C, \lambda$ and $v$ :

$$
\begin{aligned}
C & =\omega_{0}^{\prime}(\kappa)+H_{0}-\frac{E}{96 \pi^{2} \mu \kappa^{2}}+v \omega_{0}^{\prime \prime}(\kappa) E, \\
\lambda C & =\kappa+\lambda, \\
\nu C & =\frac{1}{96 \pi^{2} \mu \kappa}+\frac{1}{4} \lambda+v \omega_{0}^{\prime}(\kappa) .
\end{aligned}
$$

Elimination of $\lambda$ and $v$ gives one equation for $C$ :

$$
\begin{aligned}
& {\left[C-\omega_{0}^{\prime}(\kappa)\right]^{2}=\left[H_{0}-\frac{E}{96 \pi^{2} \mu \kappa^{2}}\right]\left[C-\omega_{0}^{\prime}(\kappa)\right]+} \\
& +\omega_{0}^{\prime \prime}(\kappa) E\left[\frac{\kappa}{4(C-1)}+\frac{1}{96 \pi^{2} \mu \kappa}\right] .
\end{aligned}
$$

It may be seen from equation (1.46) that for linear waves with $E \rightarrow 0$ and $H_{0} \rightarrow 0$ there is a double root $C=C_{0}=\omega_{0}{ }^{\prime}(\kappa)$ corresponding to the linear group velocity. For non-linear waves $H_{0}$ and $E$ are of order $\varepsilon^{2}$ and then we have two roots $C_{1}$ and $C_{2}$ near $C_{0}=\omega_{0}{ }^{\prime}(\kappa)$ and one root $C_{3}$ near +1 . The three characteristic velocities $C_{i},(i=1,2,3)$, are approximately found to be:

$$
\begin{aligned}
C_{1,2} & =C_{0} \pm \sqrt{\omega_{0}^{\prime \prime}(\kappa) E\left\{\frac{\kappa}{4\left(C_{0}-1\right)}+\frac{1}{96 \pi^{2} \mu \kappa}\right\}}+O\left(\varepsilon^{\frac{3}{2}}\right)= \\
& =C_{0} \pm \frac{1}{2} \sqrt{ } E+O\left(\varepsilon^{\frac{3}{2}}\right), \\
C_{3} & =1+\frac{\kappa E \omega_{0}^{\prime \prime}(\kappa)}{4\left(1-C_{0}\right)^{2}}+O\left(\varepsilon^{3}\right)=1-\frac{E}{24 \pi^{2} \mu \kappa^{2}}+O\left(\varepsilon^{3}\right) .
\end{aligned}
$$

The characteristic velocities are real and hence we are dealing with a hyperbolic system of equations that may be written in characteristic form as follows:

$$
\frac{\partial \kappa}{\partial t}+C_{i} \frac{\partial \kappa}{\partial x}+\lambda_{i}\left\{\frac{\partial H_{0}}{\partial t}+C_{i} \frac{\partial H_{0}}{\partial x}\right\}+v_{i}\left\{\frac{\partial E}{\partial t}+C_{i} \frac{\partial E}{\partial x}\right\}=0
$$

$$
(i=1,2,3)
$$


with multipliers $\lambda_{i}$ and $v_{i}$ given by

$$
\begin{aligned}
\lambda_{i} & =\frac{\kappa}{C_{i}-1}, \\
v_{i} & =\frac{1}{C_{i}-C_{o}}\left[\frac{1}{96 \pi^{2} \mu \kappa}+\frac{\kappa}{4\left(C_{i}-1\right)}\right] .
\end{aligned}
$$

Note that the characteristic velocities do not depend on the mean waveheight $H_{0}(x, t)$ to the present order of approximation. Also we observe that for $i=1$ and $i=2$ (corresponding to the characteristic velocities $C_{1}$ and $C_{2}$ near the linear group velocity $\left.C_{0}=\omega_{0}{ }^{\prime}(\kappa)\right)$ the multipliers $v_{1}$ and $v_{2}$ are large of order $\varepsilon^{-1}$ compared to $\lambda_{1}$ and $\lambda_{2}$. This follows from

$$
\frac{1}{C_{1,2}-C_{0}}=O(1 / \varepsilon)
$$

By virtue of $E=O\left(\varepsilon^{2}\right)$ and $H_{0}=O\left(\varepsilon^{2}\right)$ the terms containing $H_{0}$ in equation (1.49) can be neglected for $i=1$ and $i=2$. In fact they are of the same order of magnitude as terms omitted in earlier stages of the analysis. Hence to the present order of approximation we get two equations involving the functions $\kappa(x, t)$ and $E(x, t)$ :

$$
\begin{aligned}
& \frac{\partial \kappa}{\partial t}+\left(C_{0}+\frac{1}{2} \sqrt{ } E\right) \frac{\partial \kappa}{\partial x}-\frac{1}{48 \pi^{2} \mu \kappa \sqrt{ } E}\left[\frac{\partial E}{\partial t}+\left(C_{0}+\frac{1}{2} \sqrt{ } E\right) \frac{\partial E}{\partial x}\right]=0, \\
& \frac{\partial \kappa}{\partial t}+\left(C_{0}-\frac{1}{2} \sqrt{ } E\right) \frac{\partial \kappa}{\partial x}+\frac{1}{48 \pi^{2} \mu \kappa \sqrt{ } E}\left[\frac{\partial E}{\partial t}+\left(C_{0}-\frac{1}{2} \sqrt{ } E\right) \frac{\partial E}{\partial x}\right]=0 .
\end{aligned}
$$

By addition and subtraction of these equations we find successively:

$$
\begin{aligned}
& \frac{\partial \kappa}{\partial t}+C_{0} \frac{\partial \kappa}{\partial x}-\frac{1}{96 \pi^{2} \mu \kappa} \frac{\partial E}{\partial x}=0, \\
& \frac{\partial E}{\partial t}+\frac{\partial}{\partial x}\left(C_{0} E\right)=0 .
\end{aligned}
$$

Introducing a new dependent variable $C_{0}(x, t)$ we obtain the set of equations:

$$
\begin{aligned}
& \frac{\partial C_{0}}{\partial t}+C_{0} \frac{\partial C_{0}}{\partial x}+\frac{1}{4} \frac{\partial E}{\partial x}=0, \\
& \frac{\partial E}{\partial t}+\frac{\partial}{\partial x}\left(C_{0} E\right)=0 .
\end{aligned}
$$


This remarkable result shows that for cnoidal waves the energy density $E(x, t)$ and its propagation velocity $C_{0}(x, t)$ satisfy the one-dimensional unsteady equations for a compressible fluid:

$$
\begin{array}{ll}
\frac{\partial u}{\partial t}+u \frac{\partial u}{\partial x}+\frac{1}{\varrho} \frac{\partial p}{\partial x}=0, & \text { (momentum equation) } \\
\frac{\partial \varrho}{\partial t}+\frac{\partial}{\partial x}(\varrho u)=0, & \text { (continuity equation) }
\end{array}
$$

with an adiabatic pressure-density relation $p=\frac{1}{8} \varrho^{2}$. These equations are also similar to the first-order non-linear shallow water equations. An important feature of equations (1.54) and (1.55) is that the two unknown functions have a different order of magnitude: $C_{0}(x, t)$ is of order unity and $E(x, t)$ is of order $\varepsilon^{2}$. Based on this fact we give an asymptotic method of solution of equations (1.54) and (1.55) in the following section.

\section{I.7 An asymptotic solution of the initial value problem for slowly varying wavetrains}

Equations (1.54) and (1.55) are identical to the equations of one-dimensional unsteady gas dynamics and in order to stress this analogy they are rewritten as:

$$
\begin{aligned}
& \frac{\partial u}{\partial t}+u \frac{\partial u}{\partial x}+\varepsilon^{2} \frac{\partial \varrho}{\partial x}=0, \\
& \frac{\partial \varrho}{\partial t}+u \frac{\partial \varrho}{\partial x}+\varrho \frac{\partial u}{\partial x}=0,
\end{aligned}
$$

with $\varrho(x, t)$ and $u(x, t)$ of order unity.

Introduction of a new dependent variable $v(x, t)$ by means of

$$
v(x, t)=2 \varepsilon \sqrt{\varrho(x, t)},
$$

leads to the equations

$$
\begin{aligned}
& \frac{\partial u}{\partial t}+u \frac{\partial u}{\partial x}+\frac{1}{2} v \frac{\partial v}{\partial x}=0 \\
& \frac{\partial v}{\partial t}+u \frac{\partial v}{\partial x}+\frac{1}{2} v \frac{\partial u}{\partial x}=0 .
\end{aligned}
$$


Considering $x$ and $t$ as functions of $u$ and $v$, equations (1.58) and (1.59) transform into

$$
\begin{array}{r}
-\frac{\partial x}{\partial v}+u \frac{\partial t}{\partial v}-\frac{1}{2} v \frac{\partial t}{\partial u}=0 \\
\frac{\partial x}{\partial u}+\frac{1}{2} v \frac{\partial t}{\partial v}-u \frac{\partial t}{\partial u}=0 .
\end{array}
$$

Introducing a function $\phi(u, v)$ by

$$
\begin{aligned}
& \frac{\partial \phi}{\partial v}=-\frac{1}{2} v t, \\
& \frac{\partial \phi}{\partial u}=x-u t,
\end{aligned}
$$

then equation (1.60) is satisfied. From equation (1.61) follows the equation for $\phi(u, v)$ :

$$
\frac{\partial^{2} \phi}{\partial v^{2}}+\frac{1}{v} \frac{\partial \phi}{\partial v}-\frac{\partial^{2} \phi}{\partial u^{2}}=0
$$

which is a two-dimensional axisymmetric wave equation.

It is possible now to formulate an initial value problem for slowly varying wavetrains. Suppose that for $t=0$ the energy density $E(x, 0)$ and the wavenumber distribution $\kappa(x, 0)$ are given as smooth bounded functions of $x$. Then also $C_{0}(x, 0)$ is fixed. In terms of equations (1.58) and (1.59) this means that at $t=0$ the functions $u(x, 0)$ and $v(x, 0)$ are given. Note that both $u(x, 0)$ and $v(x, 0)$ are positive and that $u(x, 0)$ is of order unity whereas $v(x, 0)$ is of order $\varepsilon$. Elimination of $x$ from $u=u(x, 0)$ and $v=v(x, 0)$ gives a smooth arc in the $u, v$-plane bounded by the extremal values $u_{\max }$ and $u_{\min }$ of $u(x, 0)$. This curve is denoted by $v=\varepsilon g(u)$ and has a distance of order $\varepsilon$ from the $u$-axis because of $v=O(\varepsilon)$. (See fig. 2).

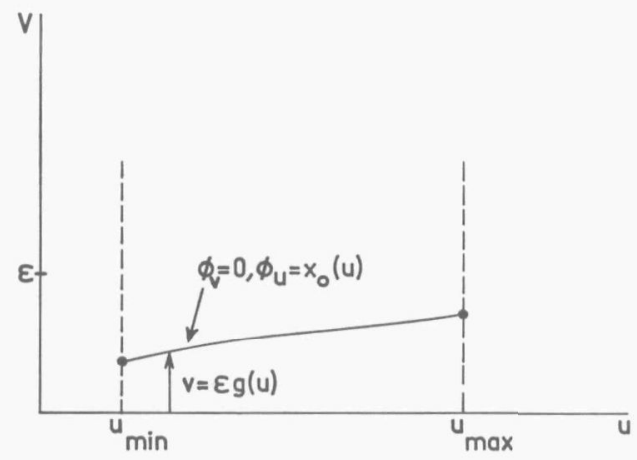

Fig. 2. 
In order to give boundary conditions for $\phi(u, v)$ we observe that on $v=\varepsilon g(u)$ we have $t=0$ and $x$ is a given function of $u, x=x_{0}(u)$ say. From equations (1.62) and (1.63) follow the boundary conditions for $\phi(u, v)$ on $v=\varepsilon g(u)$ :

$$
\begin{aligned}
& {\left[\frac{\partial \Phi}{\partial u}\right]_{v=\varepsilon g(u)}=x_{0}(u),} \\
& {\left[\frac{\partial \Phi}{\partial v}\right]_{v=\varepsilon g(u)}=0 .}
\end{aligned}
$$

In this way a boundary value problem for equation (1.64) is obtained bearing some resemblance to the axisymmetric slender body theory in supersonic aerodynamics. As we are only interested in solutions for small values of $v$, i.e. $v=O(\varepsilon)$, this problem can be solved approximately in a simple way by replacing the term $\phi_{u u}$ in equation (1.64) by $x_{0}{ }^{\prime}(u)$ for small values of $v$.Then equation (1.64) reduces to an ordinary differential equation in $v$ with the solution

$$
\begin{aligned}
& \phi_{0}(u, v)=X_{0}(u)+\frac{1}{2} \varepsilon^{2} g^{2}(u) X_{0}^{\prime \prime}(u) \ln \left[\frac{\varepsilon g(u)}{v}\right]+ \\
& +\frac{1}{4} X_{0}^{\prime \prime}(u)\left[v^{2}-\varepsilon^{2} g^{2}(u)\right],
\end{aligned}
$$

where $X_{0}(u)$ is defined by:

$$
X_{0}^{\prime}(u)=x_{0}(u)
$$

Notice that this asymptotic solution of equation (1.64) satisfies boundary conditions (1.65) and (1.66) exactly.

In order to justify this asymptotic solution we proceed with the next approximation. The remainder function $\psi(u, v)$ defined by

$$
\phi(u, v)=\phi_{0}(u, v)+\psi(u, v),
$$

satisfies the differential equation

$$
\begin{aligned}
& \frac{\partial^{2} \psi}{\partial v^{2}}+\frac{1}{v} \frac{\partial \psi}{\partial v}-\frac{\partial^{2} \psi}{\partial u^{2}}=\frac{\partial^{2}}{\partial u^{2}}\left[\frac{1}{2} \varepsilon^{2} g^{2}(u) X_{0}^{\prime \prime}(u) \ln \left\{\frac{\varepsilon g(u)}{v}\right\}+\right. \\
& \left.+\frac{1}{4} X_{0}^{\prime \prime}(u)\left\{v^{2}-\varepsilon^{2} g^{2}(u)\right\}\right]
\end{aligned}
$$

with boundary conditions 


$$
\begin{aligned}
& {\left[\frac{\partial \psi}{\partial u}\right]_{v=\varepsilon g(u)}=0,} \\
& {\left[\frac{\partial \psi}{\partial v}\right]_{v=\varepsilon g(u)}=0 .}
\end{aligned}
$$

Equation (1.67) for $\psi(u, v)$ has the form:

$$
\frac{\partial^{2} \psi}{\partial v^{2}}+\frac{1}{v} \frac{\partial \psi}{\partial v}-\frac{\partial^{2} \psi}{\partial u^{2}}=\varepsilon^{2} f_{1}(u)+\varepsilon^{2} \ln \varepsilon \cdot f_{2}(u)+v^{2} f_{3}(u)+\varepsilon^{2} \ln v \cdot f_{4}(u)
$$

with functions $f_{i}(u),(i=1,2,3,4)$ of order of magnitude one. Assuming that $\psi_{u u}$ is small compared to the terms of the righthand side of equation (1.70), the term $\psi_{u u}$ may be omitted from equation (1.70). The resulting ordinary differential equation has the solution

$$
\begin{aligned}
& \psi_{0}(u, v)=A(u) \ln v+B(u)+\frac{1}{4} v^{2}\left[\varepsilon^{2} f_{1}(u)+\varepsilon^{2} \ln \varepsilon \cdot f_{2}(u)\right]+ \\
& +\frac{1}{16} v^{4} f_{3}(u)+\frac{1}{4} \varepsilon^{2} f_{4}(u) \cdot v^{2}(\ln v-1)
\end{aligned}
$$

It is easy to see that by virtue of boundary conditions (1.68) and (1.69) $A(u)$ and $B(u)$ are of order $\varepsilon^{4} \ln \varepsilon$ and $\varepsilon^{4} \ln ^{2} \varepsilon$ respectively. Hence in the region where $v$ is of order $\varepsilon$, the second approximation $\psi_{0}(u, v)$ is of order $\varepsilon^{4} \ln ^{2} \varepsilon$. This justifies the neglect of the term $\psi_{u u}$ in equation (1.70).

LightHILL [13,14], who used Whitham's averaged Lagrangian principle, also arrived at the two-dimensional axisymmetric wave equation (1.64) in his study of a restricted class of non-linear dispersive wave problems, i.e. problems leading to equations containing the wavenumber and frequency only. The analysis of this chapter shows that for cnoidal waves (and also for Boussinesq waves as will appear in the next chapter) a similar theory as expounded by LigHTHILL is possible and hence for a detailed study of boundary value problems arising from equation (1.64) in the case of an initially given slowly varying wavetrain and also for various kinds of wavemakers we refer to LighthiLL $[13,14]$. 
Chapter II

\section{BOUSSINESQ WAVES}

\section{II.1 The Boussinesq equations}

The Boussinesq equations for shallow water waves are

$$
\begin{aligned}
& \frac{\partial \bar{h}}{\partial \bar{t}}+\frac{\partial}{\partial \bar{x}}(\bar{u} \bar{h})+\frac{\partial}{\partial \bar{y}}(\bar{v} \bar{h})=0, \\
& \frac{\partial \bar{u}}{\partial \bar{t}}+\bar{u} \frac{\partial \bar{u}}{\partial \bar{x}}+\bar{v} \frac{\partial \bar{u}}{\partial \bar{y}}+g \frac{\partial \bar{h}}{\partial \bar{x}}+\frac{1}{3} \bar{h}_{0} \frac{\partial^{3} \bar{h}}{\partial \bar{x} \partial \bar{t}^{2}}=0, \\
& \frac{\partial \bar{v}}{\partial \bar{t}}+\bar{u} \frac{\partial \bar{v}}{\partial \bar{x}}+\bar{v} \frac{\partial \bar{v}}{\partial \bar{y}}+g \frac{\partial \bar{h}}{\partial \bar{y}}+\frac{1}{3} \bar{h}_{0} \frac{\partial^{3} \bar{h}}{\partial \bar{y} \partial \bar{t}^{2}}=0,
\end{aligned}
$$

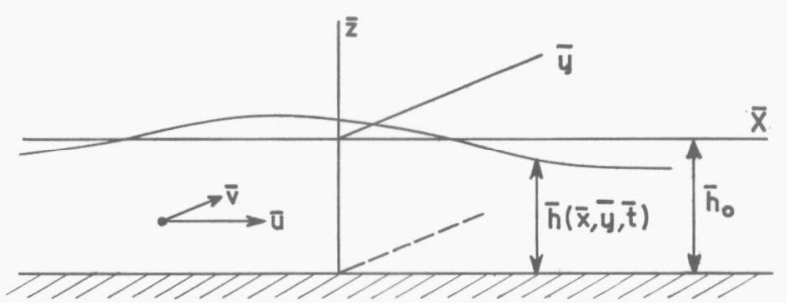

Fig. 3.

where $\bar{u}(\bar{x}, \bar{y}, \bar{t})$ and $\bar{v}(\bar{x}, \bar{y}, \bar{t})$ denote the horizontal components of the velocity in $\bar{x}-$ and $\bar{y}$-direction respectively, $\bar{h}(\bar{x}, \bar{y}, \bar{t})$ denotes the total depth and $\bar{h}_{0}$ is the constant undisturbed depth. As usual in the shallow water approximation the $\bar{z}$-dependence and the vertical velocity are absent in the governing equations. A derivation of the Boussinesq equations may be found in WhITHAM [12].

Analogous to the case of the cnoidal waves, equations (2.1), (2.2) and (2.3) are valid for small values of two non-dimensional parameters $\varepsilon$ and $\mu$ defined as

$$
\varepsilon=\frac{a}{\bar{h}_{0}}, \quad \mu=\frac{\bar{h}_{0}^{2}}{3 \lambda_{0}^{2}}
$$

where $a$ is a typical amplitude and $\lambda_{0}$ a typical wavelength. After introduction of the non-dimensional variables $u, v, h, x, y$ and $t$ : 


$$
\begin{aligned}
& x=\frac{\bar{x}}{\lambda_{0}}, \quad y=\frac{\bar{y}}{\lambda_{0}}, \quad h=\frac{\bar{h}}{\bar{h}_{0}}, \\
& u=\frac{\bar{u}}{\sqrt{g \bar{h}_{0}}}, \quad v=\frac{\bar{v}}{\sqrt{g \bar{h}_{0}}}, \quad t=\frac{\sqrt{g \bar{h}_{0}}}{\lambda_{0}},
\end{aligned}
$$

the Boussinesq equations transform into

$$
\begin{aligned}
& \frac{\partial h}{\partial t}+\frac{\partial}{\partial x}(u h)+\frac{\partial}{\partial y}(v h)=0 \\
& \frac{\partial u}{\partial t}+u \frac{\partial u}{\partial x}+v \frac{\partial u}{\partial y}+\frac{\partial h}{\partial x}+\mu \frac{\partial^{3} h}{\partial x \partial t^{2}}=0, \\
& \frac{\partial v}{\partial t}+u \frac{\partial v}{\partial x}+v \frac{\partial v}{\partial y}+\frac{\partial h}{\partial y}+\mu \frac{\partial^{3} h}{\partial y \partial t^{2}}=0 .
\end{aligned}
$$

From equations (2.5) and (2.6) follows the additional relation

$$
\frac{\partial u}{\partial y}=\frac{\partial v}{\partial x}
$$

expressing the irrotationality of the flow. In equations (2.4), (2.5), (2.6) and (2.7) we have wavelengths and periods of order unity, velocities $u$ and $v$ of order $\varepsilon$ and total depth $h=1+O(\varepsilon)$.

For $\mu=0$ the equations reduce to the well-known first-order non-linear shallow water equations for constant depth that display no dispersion. Linearization with respect to $\varepsilon$ only leads to the linear Boussinesq equations

$$
\begin{aligned}
& \frac{\partial h}{\partial t}+\frac{\partial u}{\partial x}+\frac{\partial v}{\partial y}=0 \\
& \frac{\partial u}{\partial t}+\frac{\partial h}{\partial x}+\mu \frac{\partial^{3} h}{\partial x \partial t^{2}}=0 \\
& \frac{\partial v}{\partial t}+\frac{\partial h}{\partial y}+\mu \frac{\partial^{3} h}{\partial y \partial t^{2}}=0
\end{aligned}
$$

Substitution of the harmonic plane wave 


$$
\begin{aligned}
& h=1+A \cos \left[2 \pi\left(\kappa_{1} x+\kappa_{2} y-\omega t\right)\right], \\
& u=\quad B_{1} \cos \left[2 \pi\left(\kappa_{1} x+\kappa_{2} y-\omega t\right)\right], \\
& v=\quad B_{2} \cos \left[2 \pi\left(\kappa_{1} x+\kappa_{2} y-\omega t\right)\right],
\end{aligned}
$$

into the linear Boussinesq equations gives a set of dispersion relations connecting the frequency $\omega$ with the wavenumbers $\kappa_{1}$ and $\kappa_{2}$ :

$$
\kappa_{1}^{2}+\kappa_{2}^{2}-\omega^{2}=4 \pi^{2} \mu \omega^{2}\left(\kappa_{1}^{2}+\kappa_{2}^{2}\right),
$$

and relating the amplitudes $A, B_{1}$ and $B_{2}$ by

$$
\omega A=\left(\kappa_{1}^{2}+\kappa_{2}^{2}\right) \frac{B_{1}}{\kappa_{1}}=\left(\kappa_{1}^{2}+\kappa_{2}^{2}\right) \frac{B_{2}}{\kappa_{2}} .
$$

In the following sections we will study the behaviour of slowly varying wavetrains governed by the non-linear Boussinesq equations (2.4), (2.5) and (2.6). At first onedimensional waves are treated, i.e. the case without $y$-dependence, then the results for the two-dimensional case will be given.

\section{II.2 Asymptotic expansions with respect to $K$ for the one-dimensional case}

Stretching the horizontal coordinate and the time by a large factor $K$ the one-dimensional Boussinesq equations become

$$
\begin{aligned}
& \frac{\partial h}{\partial t}+\frac{\partial}{\partial x}(u h)=0, \\
& \frac{\partial u}{\partial t}+u \frac{\partial u}{\partial x}+\frac{\partial h}{\partial x}+\frac{\mu}{K^{2}} \frac{\partial^{3} h}{\partial x \partial t^{2}}=0,
\end{aligned}
$$

where now $x$ and $t$ denote the stretched coordinates.

Before writing down the asymptotic representations for slowly varying wavetrains we remark that it is natural to expect that if curves $S(x, t)=$ constant are wavefronts for the waveheight $h(x, t)$, they also are wavefronts for the velocity $u(x, t)$. Hence we are looking for solutions of equations (2.13) and (2.14) which may be represented asymptotically by

$$
\begin{aligned}
& u(x, t)=U[K S(x, t), x, t]+\frac{1}{K} U_{1}[K S(x, t), x, t]+O\left(\frac{1}{K^{2}}\right), \\
& h(x, t)=H[K S(x, t), x, t]+\frac{1}{K} H_{1}[K S(x, t), x, t]+O\left(\frac{1}{K^{2}}\right) .
\end{aligned}
$$


Insertion of these two asymptotic expansions into the Boussinesq equations (2.13) and (2.14) and collecting powers of $K$ yields as terms of order $K$ :

$$
\begin{aligned}
& -\omega U_{p}+\kappa U U_{p}+\kappa H_{p}+\mu \kappa \omega^{2} H_{p p p}=0, \\
& -\omega H_{p}+\kappa U H_{p}+\kappa H U_{p}=0,
\end{aligned}
$$

with the usual notations

$$
\kappa=S_{x}, \quad \omega=-S_{t}, \quad p=K S(x, t) .
$$

The terms of order unity lead to the equations:

$$
\begin{aligned}
& (\kappa U-\omega) U_{1 p}+\kappa U_{p} U_{1}+\kappa H_{1 p}+\mu \kappa \omega^{2} H_{1 p p p}=F_{1}(p, x, t), \\
& (\kappa U-\omega) H_{1 p}+\kappa U_{p} H_{1}+\kappa\left(H U_{1}\right)_{p}=F_{2}(p, x, t),
\end{aligned}
$$

with inhomogeneous terms $F_{1}$ and $F_{2}$ defined by:

and

$$
\begin{aligned}
& F_{1}(p, x, t)=-U_{t}-U U_{x}-H_{x}-2 \omega \omega_{x} \mu H_{p p}+ \\
& +\kappa \omega_{t} \mu H_{p p}+2 \kappa \omega \mu H_{p p t}-\omega^{2} \mu H_{p p x}
\end{aligned}
$$

$$
F_{2}(p, x, t)=-H_{t}-U H_{x}-H U_{x} .
$$

At first we consider equations (2.15) and (2.16) determining $U$ and $H$ as functions of $p$. Integration with respect to $p$ gives:

$$
\begin{aligned}
& -\omega U+\frac{1}{2} \kappa U^{2}+\kappa H+\mu \kappa \omega^{2} H_{p p}=\text { constant, } \\
& -\omega H+\kappa H U=\text { constant }=\beta .
\end{aligned}
$$

Elimination of $U$ from equations (2.19) and (2.20) and integrating once again with respect to $p$ leads to an equation for $H$ only:

$$
\mu \kappa \omega^{2} H_{p}^{2}=\alpha H+\gamma+\frac{\beta^{2}}{\kappa H}-\kappa H^{2},
$$

with $\alpha(x, t), \beta(x, t)$ and $\gamma(x, t)$ as constants of integration. It is seen from equation (2.21) that $H$ is a periodic function of $p$ oscillating between those two zeroes $H_{\min }(x, t)$ and $H_{\max }(x, t)$ of the righthand side of equation (2.21) for which this righthand side is positive when $H_{\min }<H<H_{\max }$. By virtue of equation (2.20) $U$ is also periodic in 
$p$ with the same period as $H$ and from equation (2.16) it may be deduced that $H_{p}$ and $U_{p}$ are zero simultaneously and that $U$ and $H$ are oscillating in phase. $H(p, x, t)$ may be given in implicit form as:

$$
p+\dot{\delta}=\left(\mu \kappa \omega^{2}\right)^{\frac{1}{2}} \int_{H_{\min }}^{H} \frac{d H}{\left[\alpha H+\gamma+\frac{\beta^{2}}{\kappa H}-\kappa H^{2}\right]^{\frac{1}{2}}},
$$

where $\delta(x, t)$ denotes a non-essential shifting constant.

The condition that $H$ and $U$ should have a period independent of $x$ and $t$ which can be normalized to unity, gives the dispersion relation

$$
\frac{1}{2}=\left(\mu \kappa \omega^{2}\right)^{\frac{1}{2}} \int_{H_{\min }}^{H_{\max }} \frac{d H}{\left[\alpha H+\gamma+\frac{\beta^{2}}{\kappa H}-\kappa H^{2}\right]^{\frac{1}{2}}} .
$$

We observe that the first terms $U(p, x, t)$ and $H(p, x, t)$ of the asymptotic expansion for slowly varying wavetrains locally represent a uniform wave and the slow variations of the wavetrain are governed by five slowly varying parameters: $\kappa(x, t)$, $\omega(x, t), \alpha(x, t), \beta(x, t)$ and $\gamma(x, t)$. Two relations between these five functions have been obtained up to now, viz. dispersion relation (2.22) and the relation

$$
\frac{\partial \kappa}{\partial t}+\frac{\partial \omega}{\partial x}=0
$$

The three remaining equations are obtained analogous to the case of the cnoidal waves by imposing conditions of boundedness on the second terms $U_{1}(p, x, t)$ and $H_{1}(p, x, t)$ of the asymptotic expansions of $u(x, t)$ and $h(x, t)$.

\section{II.3 Three integral relations involving $U(p, x, t)$ and $H(p, x, t)$}

Equations (2.17) and (2.18) which determine $U_{1}$ and $H_{1}$, are integrated once with respect to $p$ :

$$
\begin{aligned}
& (\kappa U-\omega) U_{1}+\kappa H_{1}+\mu \kappa \omega^{2} H_{1 p p}=\int F_{1} d p+\gamma_{1}=G_{1}(p, x, t), \\
& (\kappa U-\omega) H_{1}+\kappa H U_{1}=\int F_{2} d p+\gamma_{2}=G_{2}(p, x, t),
\end{aligned}
$$

where $\gamma_{1}(x, t)$ and $\gamma_{2}(x, t)$ are constants of integration.

The lefthand side of both equation (2.24) and equation (2.25) should be bounded for large $p$ and because of the periodicity of $F_{1}$ and $F_{2}$ the integrals on the right- 
hand side of equations (2.24) and (2.25) can only be bounded for large $p$ if the integrals over one period vanish. This gives the integral relations

$$
\int_{0}^{1} F_{1}(p, x, t) d p=0
$$

and

$$
\int_{0}^{1} F_{2}(p, x, t) d p=0
$$

A third integral relation follows after integration of equations (2.24) and (2.25). Observing that a solution of the corresponding homogeneous equations is given by

$$
U_{1}^{(H)}=U_{p}, \quad H_{1}^{(H)}=H_{p},
$$

we obtain after putting

$$
H_{1}=w H_{p}
$$

and elimination of $U_{1}$ :

$$
\begin{aligned}
& -(\kappa U-\omega)^{2} w H_{p}+\kappa^{2} w H H_{p}+\mu \kappa^{2} \omega^{2} H\left(w_{p p} H_{p}+2 w_{p} H_{p p}+w H_{p p p}\right)= \\
& =\kappa H G_{1}-(\kappa U-\omega) G_{2} .
\end{aligned}
$$

Elimination of $U_{p}$ from equations (2.15) and (2.16) leads to the differential equation

$$
-(\kappa U-\omega)^{2} H_{p}+\kappa^{2} H H_{p}+\mu \kappa^{2} \omega^{2} H H_{p p p}=0
$$

and hence the terms with $w$ in equation (2.28) vanish. After some manipulations equation (2.28) may be written in the following form:

$$
\mu \kappa \omega^{2} \frac{\partial}{\partial p}\left(w_{p} H_{p}^{2}\right)=\mu \kappa \omega^{2} \frac{\partial}{\partial p}\left[H_{p} H_{1 p}-H_{p p} H_{1}\right]=H_{p} G_{1}+U_{p} G_{2} .
$$

Again the boundedness of the term between square brackets requires that the integral over one period of the inhomogeneous term is equal to zero:

$$
\int_{0}^{1}\left[H_{p} G_{1}+U_{p} G_{2}\right] d p=0 .
$$

By partial integration we find 


$$
\begin{aligned}
& \int_{0}^{1} H_{p} G_{1} d p=\int_{0}^{1} H_{p}\left\{\int F_{1} d p+\gamma_{1}\right\} d p= \\
& =\left[H\left\{\int F_{1} d p+\gamma_{1}\right\}\right]_{p=0}^{1}-\int_{0}^{1} H F_{1} d p=-\int_{0}^{1} H F_{1} d p,
\end{aligned}
$$

and in a similar way

$$
\int_{0}^{1} U_{p} G_{2} d p=-\int_{0}^{1} U F_{2} d p
$$

Then relation (2.29) becomes:

$$
\int_{0}^{1}\left[H(p, x, t) F_{1}(p, x, t)+U(p, x, t) F_{2}(p, x, t)\right] d p=0 .
$$

The three integral relations (2.26), (2.27) and (2.30), together with dispersion relation (2.22) and equation (2.23) provide a set of five equations for the determination of the slowly varying functions $\kappa(x, t), \omega(x, t), \alpha(x, t), \beta(x, t)$ and $\gamma(x, t)$. In close analogy to section I.4 we will show in the next section that integral relations (2.26), (2.27) and (2.30) may be obtained as well by applying an averaging technique to conservative equations for one-dimensional Boussinesq waves.

\section{II.4 Conservation laws for one-dimensional Boussinesq waves}

Governing equations (2.13) and (2.14) for one-dimensional Boussinesq waves can be written in conservative form as:

$$
\begin{aligned}
& \frac{\partial}{\partial t}(h)+\frac{\partial}{\partial x}(u h)=0, \\
& \frac{\partial}{\partial t}(u)+\frac{\partial}{\partial x}\left(\frac{1}{2} u^{2}+h+\frac{\mu}{K^{2}} h_{t t}\right)=0 .
\end{aligned}
$$

Equation (2.31) expresses the conservation of mass and equation (2.32) the conservation of momentum. A third conservation law follows from addition of equations (2.31) and (2.32) multiplied by $u$ and $h$ respectively:

$$
u\left\{\frac{\partial}{\partial t}(h)+\frac{\partial}{\partial x}(u h)\right\}+h\left\{\frac{\partial}{\partial t}(u)+\frac{\partial}{\partial x}\left(\frac{1}{2} u^{2}+h+\frac{\mu}{K^{2}} h_{t t}\right)\right\}=0,
$$

or in conservative form: 


$$
\frac{\partial}{\partial t}\left[u h-\frac{\mu}{K^{2}} h_{x} h_{t}\right]+\frac{\partial}{\partial x}\left[h u^{2}+\frac{1}{2} h^{2}+\frac{\mu}{K^{2}} h h_{t t}+\frac{\mu}{2 K^{2}} h_{t}^{2}\right]=0 .
$$

From equation (2.33) the structure of integral relation (2.30) becomes apparent.

If a slowly varying wavetrain is considered locally as a uniform wavetrain with slowly varying parameters $\kappa(x, t), \omega(x, t), \alpha(x, t), \beta(x, t)$ and $\gamma(x, t)$, we can put:

$$
\begin{aligned}
& u=U(p, x, t)=U[p ; \kappa(x, t), \omega(x, t), \alpha(x, t), \beta(x, t), \gamma(x, t)], \\
& h=H(p, x, t)=H[p ; \kappa(x, t), \omega(x, t), \alpha(x, t), \beta(x, t), \gamma(x, t)],
\end{aligned}
$$

with

$$
\frac{\partial p}{\partial x}=K \kappa(x, t), \quad \frac{\partial p}{\partial t}=-K \omega(x, t)
$$

and where the periodic dependence of $U$ and $H$ on $p$ is governed by differential equations (2.15) and (2.16). Insertion of $U$ and $H$ into conservative equations (2.31), (2.32) and (2.34) leads to three equations in which the coefficient of $K$ vanishes by virtue of equations (2.15) and (2.16). The remaining parts (being of order unity) are averaged over one period in $p$ in order to get equations for the slow variations of the wavetrain. In this way we obtain from equation (2.31):

$$
\int_{0}^{1}\left[H_{t}+U H_{x}+H U_{x}\right] d p=\int_{0}^{1} F_{2} d p=0
$$

and equation (2.32) gives

$$
\begin{aligned}
& \int_{0}^{1}\left[U_{t}+U U_{x}+H_{x}+2 \mu \omega \omega_{x} H_{p p}-\mu \kappa \omega_{t} H_{p p}-2 \mu \kappa \omega H_{p p t}+\mu \omega^{2} H_{p p x}\right] d p+ \\
& +O\left(\frac{1}{K}\right)=\int_{0}^{1} F_{1} d p+O\left(\frac{1}{K}\right)=0 .
\end{aligned}
$$

The relation obtained from equation (2.34) may be written as:

$$
\int_{0}^{1}\left[H F_{1}+U F_{2}\right] d p+O\left(\frac{1}{K}\right)=0 .
$$

These integral relations are, apart from terms of order $K^{-1}$, identical with integral relations (2.26), (2.27) and (2.30). 


\section{II.5 Asymptotic expansion of $U(p, x, t)$ and $H(p, x, t)$ in powers of $\varepsilon$}

The dependence of the leading terms $U(p, x, t)$ and $H(p, x, t)$ of the asymptotic expansions of $u(x, t)$ and $h(x, t)$ on the phase function $p=K S(x, t)$ is determined by differential equations (2.15) and (2.16). Similar to cnoidal waves the slowly varying parameters $\alpha(x, t), \beta(x, t)$ and $\gamma(x, t)$, occurring in $U(p, x, t)$ and $H(p, x, t)$, have no clear physical meaning and in order to get equations in terms of amplitudes, mean waveheight and mean velocity we put

$$
\begin{aligned}
& H(p, x, t)=\bar{H}_{0}(x, t)+\bar{A}(x, t) \Phi(p, x, t), \\
& U(p, x, t)=\bar{U}_{0}(x, t)+\bar{B}(x, t) \Psi(p, x, t),
\end{aligned}
$$

with

$$
\begin{aligned}
& \bar{H}_{0}(x, t)=\frac{1}{2}\left\{H_{\max }(x, t)+H_{\min }(x, t)\right\}, \\
& \bar{U}_{0}(x, t)=\frac{1}{2}\left\{U_{\text {max }}(x, t)+U_{\min }(x, t)\right\} .
\end{aligned}
$$

Then $\bar{A}(x, t)$ and $\bar{B}(x, t)$ may be considered as amplitude functions for the waveheight and velocity respectively. The functions $\Phi(p, x, t)$ and $\Psi(p, x, t)$ are periodic in $p$ with period one. The introduction of the functions $\bar{H}_{0}(x, t)$ and $\bar{U}_{0}(x, t)$ allows us to take $\Phi(p, x, t)$ and $\Psi(p, x, t)$ as functions oscillating between -1 and +1 . Notice that $\bar{H}_{0}(x, t)$ and $\bar{U}_{0}(x, t)$ are not exactly equal to the mean waveheight and mean velocity respectively.

Substitution of (2.35) and (2.36) into equations (2.20) and (2.21) gives

$$
\begin{aligned}
& \left(-\omega+\kappa \bar{U}_{0}+\kappa \bar{B} \Psi\right)\left(\bar{H}_{0}+\bar{A} \Phi\right)=\beta, \\
& \mu \kappa \omega^{2} \bar{A}^{2} \Phi_{p}^{2}=\alpha\left(\bar{H}_{0}+\bar{A} \Phi\right)+\gamma+\frac{\beta^{2}}{\kappa\left(\bar{H}_{0}+\bar{A} \Phi\right)}-\kappa\left(\bar{H}_{0}+\bar{A} \Phi\right)^{2} .
\end{aligned}
$$

In section II. 2 we found that $H$ and $U$ are oscillating in phase and hence also $\Phi$ and $\Psi$ reach their extremal values -1 and +1 simultaneously. By making use of this fact equations (2.37) and (2.38) give rise to four algebraical equations:

$$
\begin{aligned}
& \beta=\left(\bar{H}_{0}+\bar{A}\right)\left[-\omega+\kappa\left(\bar{U}_{0}+\bar{B}\right)\right]=\left(\bar{H}_{0}-\bar{A}\right)\left[-\omega+\kappa\left(\bar{U}_{0}-\bar{B}\right)\right], \\
& \alpha\left(\bar{H}_{0}+\bar{A}\right)+\gamma+\frac{\beta^{2}}{\kappa\left(\bar{H}_{0}+\bar{A}\right)}-\kappa\left(\bar{H}_{0}+\bar{A}\right)^{2}=0, \\
& \alpha\left(\bar{H}_{0}-\bar{A}\right)+\gamma+\frac{\beta^{2}}{\kappa\left(\bar{H}_{0}-\bar{A}\right)}-\kappa\left(\bar{H}_{0}-\bar{A}\right)^{2}=0 .
\end{aligned}
$$


From the first of these relations it follows

$$
\kappa \bar{H}_{0} \bar{B}-\bar{A} \omega+\bar{A} \kappa \bar{U}_{0}=0
$$

which is one of the dispersion relations for the problem.

Solving $\alpha, \beta$ and $\gamma$ as functions of $\bar{H}_{0}, \bar{U}_{0}, \bar{A}$ and $\bar{B}$ and substituting them into equation (2.38) we find:

$$
\mu \omega^{2} \Phi_{p}^{2}=\left(1-\Phi^{2}\right)\left[1-\frac{\bar{B}^{2}\left(\bar{H}_{0}^{2}-\bar{A}^{2}\right)}{\bar{A}^{2}\left(\bar{H}_{0}+\bar{A} \Phi\right)}\right] .
$$

The order of magnitude of the parameters $\bar{A}, \bar{B}, \bar{H}_{0}$ and $\bar{U}_{0}$ is expressed by the introduction of the quantities of order unity $A, B, \eta_{1}$ and $u_{1}$ :

$$
\left.\begin{array}{l}
\bar{A}(x, t)=\varepsilon A(x, t), \bar{B}(x, t)=\varepsilon B(x, t), \\
\bar{H}_{0}(x, t)=1+\varepsilon^{2} \eta_{1}(x, t), \bar{U}_{0}(x, t)=\varepsilon^{2} u_{1}(x, t) .
\end{array}\right\}
$$

Equation (2.39) then yields

$$
\frac{B}{A}=\frac{\omega}{\kappa}+O\left(\varepsilon^{2}\right)
$$

After substitution of relations (2.41) into equation (2.40) for $\Phi$ and a straightforward expansion in powers of $\varepsilon$ and making use of equation (2.42) in order to eliminate $B$, we arrive at the following differential equation for $\Phi(p, x, t)$ :

$$
\begin{aligned}
& \mu \kappa^{2} \omega^{2} \Phi_{p}{ }^{2}=\left(1-\Phi^{2}\right)\left[\kappa^{2}-\omega^{2}+\varepsilon A \omega^{2} \Phi+\right. \\
& \left.+\varepsilon^{2}\left(2 \kappa \omega u_{1}+A^{2} \omega^{2}-A^{2} \omega^{2} \Phi^{2}+\omega^{2} \eta_{1}\right)+O\left(\varepsilon^{3}\right)\right] .
\end{aligned}
$$

This equation is the counterpart of equation (1.26) for the cnoidal waves. The treatment of this equation with Lindstedt's method in order to obtain an asymptotic expansion of $\Phi(p, x, t)$ with respect to $\varepsilon$ is completely analogous to section I.5 and will not be repeated here.

The resulting asymptotic expansion of $H(p, x, t)$ becomes:

$$
H=1+\varepsilon A \cos 2 \pi p+\varepsilon^{2} \eta_{1}+\frac{\varepsilon^{2} A^{2}}{16 \pi^{2} \mu \kappa^{2}}[\cos 4 \pi p-1]+O\left(\varepsilon^{3}\right),
$$

and the condition of periodicity in $p$ with period one is found to give rise to the dispersion relation 
$\kappa^{2}-\omega^{2}=4 \pi^{2} \mu \kappa^{2} \omega^{2}+\varepsilon^{2}\left[-2 \omega \kappa u_{1}-\frac{1}{2} A^{2} \omega^{2}-\omega^{2} \eta_{1}+\frac{3 A^{2} \omega^{2}}{32 \pi^{2} \mu \kappa^{2}}\right]+O\left(\varepsilon^{3}\right)$.

The corresponding asymptotic expansion of $U(p, x, t)$ is obtained by using equation (2.20) and expanding in powers of $\varepsilon$ :

$U=\varepsilon \frac{A \omega}{\kappa} \cos 2 \pi p+\varepsilon^{2}\left[u_{1}+\left(\frac{A^{2} \omega}{2 \kappa}-\frac{A^{2} \omega}{16 \pi^{2} \mu \kappa^{3}}\right)(1-\cos 4 \pi p)\right]+O\left(\varepsilon^{3}\right)$.

After introduction of the mean waveheight $H_{0}(x, t)$ by means of

$$
H_{0}=\varepsilon^{2}\left[\eta_{1}-\frac{A^{2}}{16 \pi^{2} \mu \kappa^{2}}\right]
$$

the asymptotic series for $H(p, x, t)$ becomes:

$$
H=1+H_{0}+\varepsilon A \cos 2 \pi p+\frac{\varepsilon^{2} A^{2}}{16 \pi^{2} \mu \kappa^{2}} \cos 4 \pi p+O\left(\varepsilon^{3}\right)
$$

and by introducing the mean velocity $U_{0}(x, t)$ :

$$
U_{0}=\varepsilon^{2}\left[u_{1}+\frac{A^{2} \omega}{2 \kappa}-\frac{A^{2} \omega}{16 \pi^{2} \mu \kappa^{3}}\right]
$$

we get for $U(p, x, t)$ the asymptotic series

$$
U=U_{0}+\varepsilon \frac{A \omega}{\kappa} \cos 2 \pi p+\varepsilon^{2}\left[\frac{A^{2} \omega}{16 \pi^{2} \mu \kappa^{3}}-\frac{A^{2} \omega}{2 \kappa}\right] \cos 4 \pi p+O\left(\varepsilon^{3}\right) .
$$

Dispersion relation (2.45) transforms into

$\kappa^{2}-\omega^{2}=4 \pi^{2} \mu \kappa^{2} \omega^{2}-2 \omega \kappa U_{0}-\omega^{2} H_{0}+\varepsilon^{2}\left[\frac{1}{2} A^{2} \omega^{2}-\frac{3 A^{2} \omega^{2}}{32 \pi^{2} \mu \kappa^{2}}\right]+O\left(\varepsilon^{3}\right)$,

which may be written more conveniently as

$$
\omega=\omega_{0}(\kappa)+\frac{H_{0} \omega_{0}^{3}}{2 \kappa^{2}}+\frac{U_{0} \omega_{0}^{2}}{\kappa}-\frac{\omega_{0}^{3} E}{4 \kappa^{2}}\left[1-\frac{3}{16 \pi^{2} \mu \kappa^{2}}\right]+O\left(\varepsilon^{3}\right),
$$

where we have introduced the 'energy density' $E=\varepsilon^{2} A^{2}$ and where 


$$
\omega_{0}(\kappa)=\frac{\kappa}{\sqrt{1+4 \pi^{2} \mu \kappa^{2}}} .
$$

The slow variations of the wavetrain are governed by the five slowly varying functions $\omega(x, t), \kappa(x, t), A(x, t), H_{0}(x, t)$ and $U_{0}(x, t)$. Dispersion relation (2.50) together with the equation

$$
\frac{\partial \kappa}{\partial t}+\frac{\partial \omega}{\partial x}=0
$$

are two equations relating these five unknown functions. In the next section we will obtain the three remaining equations by insertion of asymptotic expansions (2.47) and (2.48) into the three integral relations (2.26), (2.27) and (2.30) satisfied by $U(p, x, t)$ and $H(p, x, t)$.

\section{II.6 Reduction of the integral relations}

Integral relation (2.26) is written as:

$$
\begin{aligned}
& \frac{\partial}{\partial t} \int_{0}^{1} U d p+\frac{1}{2} \frac{\partial}{\partial x} \int_{0}^{1} U^{2} d p+\frac{\partial}{\partial x} \int_{0}^{1} H d p+ \\
& +\mu\left(2 \omega \omega_{x}-\kappa \omega_{t}\right) \int_{0}^{1} H_{p p} d p-2 \kappa \omega \mu \int_{0}^{1} H_{p p t} d p+\mu \omega^{2} \int_{0}^{1} H_{p p x} d p=0 .
\end{aligned}
$$

Because of the periodicity of $H_{p}, H_{p t}$ and $H_{p x}$ the last three integrals vanish and substitution of the asymptotic expansions (2.47) and (2.48) for $U(p, x, t)$ and $H(p, x, t)$ gives:

$$
\frac{\partial U_{0}}{\partial t}+\frac{\partial}{\partial x}\left[H_{0}+\frac{E \omega_{0}^{2}}{4 \kappa^{2}}\right]+O\left(\varepsilon^{4}\right)=0
$$

Integral relation (2.27) is given by

$$
\frac{\partial}{\partial t} \int_{0}^{1} H d p+\frac{\partial}{\partial x} \int_{0}^{1} U H d p=0
$$

and leads to the equation:

$$
\frac{\partial H_{0}}{\partial t}+\frac{\partial}{\partial x}\left[U_{0}+\frac{E \omega_{0}}{2 \kappa}\right]+O\left(\varepsilon^{4}\right)=0 .
$$


Integral relation (2.30) reads:

$$
\begin{aligned}
& \frac{\partial}{\partial t} \int_{0}^{1} U H d p+\frac{\partial}{\partial x} \int_{0}^{1} U^{2} H d p+\frac{1}{2} \frac{\partial}{\partial x} \int_{0}^{1} H^{2} d p+ \\
& +\mu\left(2 \omega \omega_{x}-\kappa \omega_{t}\right) \int_{0}^{1} H H_{p p} d p-2 \mu \kappa \omega \int_{0}^{1} H H_{p p t} d p+ \\
& +\mu \omega^{2} \int_{0}^{1} H H_{p p x} d p=0,
\end{aligned}
$$

and yields after substitution of the expansions of $U(p, x, t)$ and $H(p, x, t)$ and using equation (2.52) in order to eliminate $U_{0}$ and $H_{0}$ :

$$
\begin{aligned}
& \frac{\partial}{\partial t}\left(\frac{A^{2} \omega}{2 \kappa}\right)+\frac{\partial}{\partial x}\left(\frac{A^{2} \omega^{2}}{4 \kappa^{2}}\right)+\frac{\partial}{\partial x}\left(\frac{1}{4} A^{2}\right)+ \\
& +2 \pi^{2} \mu\left[\left(\kappa \omega_{t}-2 \omega \omega_{x}\right) A^{2}+2 \kappa \omega A A_{t}-\omega^{2} A A_{x}\right]+O\left(\varepsilon^{2}\right)=0 .
\end{aligned}
$$

The term between square brackets in equation (2.54) is equal to

$$
\frac{\partial}{\partial t}\left(A^{2} \kappa \omega\right)-\frac{\partial}{\partial x}\left(\frac{1}{2} A^{2} \omega^{2}\right)
$$

and using dispersion relation (2.50) equation (2.54) is transformed into

$$
\frac{\partial}{\partial t}\left(\frac{A^{2} \kappa}{\omega_{0}}\right)+\frac{\partial}{\partial x}\left(\frac{A^{2} \omega_{0}^{2}}{\kappa^{2}}\right)+O\left(\varepsilon^{2}\right)=0 .
$$

Using the linear group velocity $C_{0}(\kappa)$ defined by

$$
C_{0}(\kappa)=\frac{d \omega_{0}}{d \kappa}=\frac{\omega_{0}^{3}(\kappa)}{\kappa^{3}},
$$

equation (2.55) may be written as

$$
\frac{\partial}{\partial t}\left(\frac{E \kappa}{\omega_{0}}\right)+\frac{\partial}{\partial x}\left(C_{0} \frac{E \kappa}{\omega_{0}}\right)+O\left(\varepsilon^{4}\right)=0 .
$$

By virtue of the relation

$$
\begin{aligned}
& \frac{\partial}{\partial t}\left(\frac{\kappa}{\omega_{0}}\right)+C_{0} \frac{\partial}{\partial x}\left(\frac{\kappa}{\omega_{0}}\right)=\left[\frac{1}{\omega_{0}}-\frac{\kappa \omega_{0}^{\prime}(\kappa)}{\omega_{0}^{2}}\right]\left[\frac{\partial \kappa}{\partial t}+\omega_{0}^{\prime}(\kappa) \frac{\partial \kappa}{\partial x}\right]= \\
& =-\left[\frac{1}{\omega_{0}}-\frac{\kappa \omega_{0}^{\prime}(\kappa)}{\omega_{0}^{2}}\right] \frac{\partial}{\partial x}\left\{\omega-\omega_{0}(\kappa)\right\}=O\left(\varepsilon^{2}\right)
\end{aligned}
$$


equation (2.56) is equivalent to the same order of approximation with:

$$
\frac{\partial E}{\partial t}+\frac{\partial}{\partial x}\left(C_{0} E\right)+O\left(\varepsilon^{4}\right)=0 .
$$

Equations (2.52), (2.53) and (2.57) complete the set of five equations for the determination of $\kappa, \omega, A, H_{0}$ and $U_{0}$. They may be given a physical interpretation as follows: equation (2.52) is an averaged momentum equation with a correction term proportional to $E$ accounting for the wave propagation, equation (2.53) is an averaged continuity equation and equation (2.57) expresses the conservation of averaged energy $E$ propagating with linear group velocity $C_{0}$ within this order of approximation. Notice that equation (2.57) is identical with energy equation (1.43) for cnoidal waves.

In the next section these equations are investigated further and it will appear that they can be reduced to a set of two equations for $\kappa$ and $E$ only, identical with the final equations (1.54) and (1.55) for the cnoidal waves of Chapter I.

\section{II.7 Investigation of the equations for $\kappa, \omega, E, H_{0}$ and $U_{0}$}

The set of five equations for $\kappa, \omega, E, H_{0}$ and $U_{0}$ is reduced to a set of four equations by eliminating $\omega$. Differentiation of dispersion relation (2.50) with respect to $x$ and using equation (2.51) leads to the equation:

$$
\begin{aligned}
& \frac{\partial \kappa}{\partial t}+C_{0} \frac{\partial \kappa}{\partial x}+\alpha_{1}(\kappa) \frac{\partial E}{\partial x}+\alpha_{1}^{\prime}(\kappa) E \frac{\partial \kappa}{\partial x}+\alpha_{2}(\kappa) \frac{\partial H_{0}}{\partial x}+ \\
& +\alpha_{2}^{\prime}(\kappa) H_{0} \frac{\partial \kappa}{\partial x}+\alpha_{3}(\kappa) \frac{\partial U_{0}}{\partial x}+\alpha_{3}^{\prime}(\kappa) U_{0} \frac{\partial \kappa}{\partial x}=0,
\end{aligned}
$$

where the prime denotes differentiation to $\kappa$ and where we have introduced the abbreviations $\alpha_{1}(\kappa), \alpha_{2}(\kappa)$ and $\alpha_{3}(\kappa)$ :

$$
\begin{aligned}
& \alpha_{1}(\kappa)=\frac{\omega_{0}^{3}}{4 \kappa^{2}}\left(\frac{3}{16 \pi^{2} \mu \kappa^{2}}-1\right), \\
& \alpha_{2}(\kappa)=\frac{\omega_{0}^{3}}{2 \kappa^{2}}, \quad \alpha_{3}(\kappa)=\frac{\omega_{0}^{2}}{\kappa} .
\end{aligned}
$$

In order to find the characteristic velocities we add equations (2.52), (2.53) and (2.57) multiplied by factors $\lambda, \sigma$ and $v$ respectively to equation (2.58). The requirement that $\kappa, E, H_{0}$ and $U_{0}$ should be differentiated in the same characteristic direction $C$ leads to four algebraical equations for $C, \lambda, \sigma$ and $v$ : 


$$
\begin{aligned}
C & =C_{0}+v E C_{0}^{\prime}+\alpha_{1}^{\prime} E+\alpha_{2}^{\prime} H_{0}+\alpha_{3}^{\prime} U_{0}+\lambda \beta^{\prime} E+\sigma \gamma^{\prime} E, \\
\nu C & =\alpha_{1}+\lambda \beta+\sigma \gamma+\nu C_{0}, \\
\lambda C & =\sigma+\alpha_{3}, \\
\sigma C & =\lambda+\alpha_{2},
\end{aligned}
$$

with the abbreviations $\beta(\kappa)$ and $\gamma(\kappa)$ :

$$
\beta(\kappa)=\frac{\omega_{0}^{2}}{4 \kappa^{2}}, \quad \gamma(\kappa)=\frac{\omega_{0}}{2 \kappa} .
$$

Elimination of $v, \lambda$ and $\sigma$ yields an equation for $C$ only:

$$
\begin{aligned}
& \left(C-C_{0}\right)^{2}=\left(C-C_{0}\right)\left[\alpha_{1}^{\prime} E+\alpha_{2}^{\prime} H_{0}+\alpha_{3}^{\prime} U_{0}+\right. \\
& \left.+\frac{E}{C^{2}-1}\left\{\beta^{\prime}\left(\alpha_{2}+\alpha_{3} C\right)+\gamma^{\prime}\left(\alpha_{3}+\alpha_{2} C\right)\right\}\right]+ \\
& +E C_{0}^{\prime}\left[\alpha_{1}+\frac{\beta\left(\alpha_{2}+\alpha_{3} C\right)+\gamma\left(\alpha_{3}+\alpha_{2} C\right)}{C^{2}-1}\right] .
\end{aligned}
$$

For linear waves with $E, H_{0}$ and $U_{0}$ infinitesimally small there is a double root $C=C_{0}=\omega_{0}^{\prime}(\kappa)$ corresponding to the linear group velocity. For non-linear waves with $\varepsilon$ small it is seen from equation (2.59) that two characteristic velocities $C_{1}$ and $C_{2}$ lie near $C_{0}$ and two other ones $C_{3}$ and $C_{4}$ near -1 and +1 respectively. Approximately we have:

$$
\begin{aligned}
C_{1,2} & =C_{0} \pm\left[E C_{0}^{\prime}\left\{\alpha_{1}+\frac{\beta\left(\alpha_{2}+\alpha_{3} C_{0}\right)+\gamma\left(\alpha_{3}+\alpha_{2} C_{0}\right)}{C_{0}^{2}-1}\right\}\right]^{\frac{1}{2}}+O\left(\varepsilon^{\frac{3}{2}}\right)= \\
& =C_{0} \pm\left[\frac{E C_{0}^{\prime} \omega_{0}^{9}}{16 \kappa^{8}\left(C_{0}^{2}-1\right)}\left\{12 \frac{\kappa^{6}}{\omega_{0}^{6}}-\frac{\kappa^{4}}{\omega_{0}^{4}}+5 \frac{\kappa^{2}}{\omega_{0}^{2}}-7\right\}\right]^{\frac{1}{2}}+O\left(\varepsilon^{\frac{3}{2}}\right) .
\end{aligned}
$$

By virtue of the definition of $\omega_{0}(\kappa)$ the following inequalities hold:

$$
\begin{aligned}
& C_{0}^{\prime}(\kappa)=\omega_{0}^{\prime \prime}(\kappa)<0, \\
& C_{0}^{2}-1=\left\{\omega_{0}^{\prime}(\kappa)\right\}^{2}-1<0, \\
& \frac{\kappa}{\omega_{0}}>1 .
\end{aligned}
$$


Furthermore the polynomial of the sixth degree in $\kappa / \omega_{0}$ between square brackets in equation (2.60) is positive for $\kappa / \omega_{0}>1$ and hence the radical in equation (2.60) is real. So there are always two distinct real characteristic velocities $C_{1}$ and $C_{2}$ near $C_{0}$. The two remaining velocities $C_{3,4}$ will not be needed in the sequel. It is sufficient to notice that they are real and hence we are dealing with a purely hyperbolic system of partial differential equations which may be written in characteristic form as:

$$
\begin{aligned}
& \frac{\partial \kappa}{\partial t}+C_{i} \frac{\partial \kappa}{\partial x}+v_{i}\left[\frac{\partial E}{\partial t}+C_{i} \frac{\partial E}{\partial x}\right]+\sigma_{i}\left[\frac{\partial H_{0}}{\partial t}+C_{i} \frac{\partial H_{0}}{\partial x}\right]+ \\
& +\lambda_{i}\left[\frac{\partial U_{0}}{\partial t}+C_{i} \frac{\partial U_{0}}{\partial x}\right]=0, \quad(i=1,2,3,4)
\end{aligned}
$$

with multipliers $v_{i}, \sigma_{i}$ and $\lambda_{i}$ given by

$$
\begin{aligned}
v_{i} & =\frac{1}{C_{i}-C_{0}}\left[\alpha_{1}+\frac{1}{C_{i}^{2}-1}\left\{\beta\left(\alpha_{2}+\alpha_{3} C_{i}\right)+\gamma\left(\alpha_{3}+\alpha_{2} C_{i}\right)\right\}\right] \\
\sigma_{i} & =\frac{\alpha_{3}+\alpha_{2} C_{i}}{C_{i}^{2}-1} \\
\lambda_{i} & =\frac{\alpha_{2}+\alpha_{3} C_{i}}{C_{i}^{2}-1}
\end{aligned}
$$

Analogous to the cnoidal waves we consider only $i=1$ and $i=2$, viz. the characteristic directions near the linear group velocity $C_{0}$. For $i=1$ and $i=2$ we observe that $v_{i}$ is large of order $1 / \varepsilon$ compared to $\sigma_{i}$ and $\lambda_{i}$ and hence the terms containing $H_{0}$ and $U_{0}$ in equation (2.61) can be neglected for $i=1$ and $i=2$. Noting also that $C_{1}$ and $C_{2}$ do not depend on $H_{0}$ and $U_{0}$ within the present order of approximation we get for $i=1$ and $i=2$ a set of two equations containing $\kappa(x, t)$ and $E(x, t)$ only:

$$
\begin{aligned}
& \frac{\partial \kappa}{\partial t}+\left\{C_{0}+\sqrt{E C_{0}^{\prime} F(\kappa)}\right\} \frac{\partial \kappa}{\partial x}+\sqrt{\frac{F(\kappa)}{E C_{0}^{\prime}}}\left[\frac{\partial E}{\partial t}+\left\{C_{0}+\sqrt{E C_{0}^{\prime} F(\kappa)}\right\} \frac{\partial E}{\partial x}\right]=0, \\
& \frac{\partial \kappa}{\partial t}+\left\{C_{0}-\sqrt{E C_{0}^{\prime} F(\kappa)}\right\} \frac{\partial \kappa}{\partial x}-\sqrt{\frac{F(\kappa)}{E C_{0}^{\prime}}}\left[\frac{\partial E}{\partial t}+\left\{C_{0}-\sqrt{E C_{0}^{\prime} F(\kappa)}\right\} \frac{\partial E}{\partial x}\right]=0,
\end{aligned}
$$

where $F(\kappa)$ stands for:

$$
F(\kappa)=\frac{\omega_{0}^{9}(\kappa)}{16 \kappa^{8}\left\{C_{0}^{2}(\kappa)-1\right\}}\left[12\left(\frac{\kappa}{\omega_{0}}\right)^{6}-\left(\frac{\kappa}{\omega_{0}}\right)^{4}+5\left(\frac{\kappa}{\omega_{0}}\right)^{2}-7\right]<0 .
$$


Addition and subtraction of equations (2.62) and (2.63) gives the set of equations:

$$
\begin{aligned}
& \frac{\partial \kappa}{\partial t}+C_{0}(\kappa) \frac{\partial \kappa}{\partial x}+F(\kappa) \frac{\partial E}{\partial x}=0 \\
& \frac{\partial E}{\partial t}+\frac{\partial}{\partial x}\left[C_{0}(\kappa) E\right]=0 .
\end{aligned}
$$

Introducing a new dependent variable $C_{0}(x, t)$ equation (2.64) transforms into

$$
\frac{\partial C_{0}}{\partial t}+C_{0} \frac{\partial C_{0}}{\partial x}+f\left(C_{0}\right) \frac{\partial E}{\partial x}=0
$$

with $f\left(C_{0}\right)$ determined by

$$
f\left[C_{0}(\kappa)\right]=C_{0}^{\prime}(\kappa) F(\kappa) .
$$

To the same order of approximation equation (2.66) may be replaced by

$$
\frac{\partial C_{0}}{\partial t}+C_{0} \frac{\partial C_{0}}{\partial x}+\frac{\partial}{\partial x}\left\{f\left(C_{0}\right) E\right\}=0,
$$

because the additional term $f^{\prime}\left(C_{0}\right) E C_{0 x}$ in equation (2.67) only contributes to terms of order $\varepsilon^{\frac{3}{2}}$ in the characteristic velocities $C_{1}$ and $C_{2}$ and hence the set of equations (2.67) and (2.65) has approximately the same characteristic form as equations (2.66) and (2.65), namely

$$
\begin{aligned}
& \frac{\partial C_{0}}{\partial t}+\left\{C_{0}+\sqrt{E f\left(C_{0}\right)}\right\} \frac{\partial C_{0}}{\partial x}+\sqrt{\frac{f\left(C_{0}\right)}{E}}\left[\frac{\partial E}{\partial t}+\left\{C_{0}+\sqrt{E f\left(C_{0}\right)}\right\} \frac{\partial E}{\partial x}\right]=0, \\
& \frac{\partial C_{0}}{\partial t}+\left\{C_{0}-\sqrt{E f\left(C_{0}\right)}\right\} \frac{\partial C_{0}}{\partial x}-\sqrt{\frac{f\left(C_{0}\right)}{E}}\left[\frac{\partial E}{\partial t}+\left\{C_{0}-\sqrt{E f\left(C_{0}\right)}\right\} \frac{\partial E}{\partial x}\right]=0 .
\end{aligned}
$$

Putting finally

$$
\bar{E}(x, t)=f\left(C_{0}\right) E(x, t)
$$

we arrive at the equations

$$
\begin{aligned}
& \frac{\partial C_{0}}{\partial t}+C_{0} \frac{\partial C_{0}}{\partial x}+\frac{\partial \bar{E}}{\partial x}=0, \\
& \frac{\partial \bar{E}}{\partial t}+\frac{\partial}{\partial x}\left(C_{0} \bar{E}\right)=0 .
\end{aligned}
$$


Equation (2.69) is equivalent to equation (2.65) within the present order of approximation because any function $g(\kappa)$ satisfies:

$$
\frac{\partial}{\partial t}\{g(\kappa)\}+C_{0} \frac{\partial}{\partial x}\{g(\kappa)\}=O\left(\varepsilon^{2}\right) .
$$

implying that in energy equation (2.65) $E$ may be replaced by $E$ multiplied with any function of $\kappa$.

Equations (2.68) and (2.69) show that also for one-dimensional Boussinesq waves the unsteady gas dynamics analogy holds and hence we may refer to section I.7 for an asymptotic solution of the initial value problem for slowly varying wavetrains.

\section{II.8 Two-dimensional Boussinesq waves}

Instead of treating the two-dimensional Boussinesq equations (2.4), (2.5) and (2.6) by substitution of asymptotic series in $K$ representing slowly varying wavetrains we prefer to make use of the one-dimensional case in order to give a concise derivation of the two-dimensional results. At first we derive the uniform wavetrain solution of the two-dimensional equations. It is expected that like the one-dimensional case this uniform wavetrain solution also will represent the leading terms in the asymptotic representation for slowly varying wavetrains in the two-dimensional case. The derivation of the two-dimensional uniform wavetrain is largely parallel to the onedimensional case and will only be given briefly.

For slowly varying wavetrains the constant parameters occurring in the uniform wavetrain solution are considered as slowly varying functions and the averaging technique of conservation laws which has been given in section II.4 for one-dimensional waves is extended to two-dimensional waves in order to arrive at integral relations which after substitution of the uniform wavetrain solution finally should yield equations for the slowly varying functions. The justification of the outline given here is found by using asymptotic series in $K$ as has been done for the one-dimensional case. For two-dimensional Boussinesq waves this leads to laborious calculations that will not be given here.

The governing non-dimensional equations of two-dimensional Boussinesq waves expressed in stretched coordinates $x, y$ and $t$ read as follows:

$$
\begin{aligned}
& \frac{\partial h}{\partial t}+\frac{\partial}{\partial x}(u h)+\frac{\partial}{\partial y}(v h)=0, \\
& \frac{\partial u}{\partial t}+u \frac{\partial u}{\partial x}+v \frac{\partial u}{\partial y}+\frac{\partial h}{\partial x}+\frac{\mu}{K^{2}} \frac{\partial^{3} h}{\partial x \partial t^{2}}=0, \\
& \frac{\partial v}{\partial t}+u \frac{\partial v}{\partial x}+v \frac{\partial v}{\partial y}+\frac{\partial h}{\partial y}+\frac{\mu}{K^{2}} \frac{\partial^{3} h}{\partial y \partial t^{2}}=0,
\end{aligned}
$$


together with the additional relation

$$
\frac{\partial u}{\partial y}=\frac{\partial v}{\partial x} .
$$

The uniform wavetrain solution has the form of a plane wave

$$
u=U(p), \quad v=V(p), \quad h=H(p) .
$$

with phase function $p$ defined by

$$
p=K\left(\kappa_{1} x+\kappa_{2} y-\omega t\right) .
$$

Insertion of this uniform wavetrain into equations (2.70), (2.71), (2.72) and (2.73) gives a set of four ordinary differential equations

$$
\begin{aligned}
& -\omega H_{p}+\kappa_{1}(U H)_{p}+\kappa_{2}(V H)_{p}=0, \\
& -\omega U_{p}+\kappa_{1} U U_{p}+\kappa_{2} V U_{p}+\kappa_{1} H_{p}+\mu \kappa_{1} \omega^{2} H_{p p p}=0, \\
& -\omega V_{p}+\kappa_{1} U V_{p}+\kappa_{2} V V_{p}+\kappa_{2} H_{p}+\mu \kappa_{2} \omega^{2} H_{p p p}=0, \\
& \kappa_{2} U_{p}=\kappa_{1} V_{p} .
\end{aligned}
$$

Note that equation (2.77) also may be deduced from equations (2.75) and (2.76). Integration of equations (2.74) and (2.77) yields

$$
\begin{aligned}
& -\omega H+\kappa_{1} U H+\kappa_{2} V H=\beta, \\
& \kappa_{2} U=\kappa_{1} V+\sigma,
\end{aligned}
$$

with $\beta$ and $\sigma$ constants of integration. Integrating equation (2.75) twice with respect to $p$ and using equations (2.78) and (2.79) to eliminate $U$ and $V$ we get the equation for $H(p)$ :

$$
\mu \omega^{2} H_{p}^{2}=\alpha H+\gamma+\frac{\beta^{2}}{\left(\kappa_{1}^{2}+\kappa_{2}^{2}\right) H}-H^{2},
$$

with $\alpha$ and $\gamma$ as constants of integration. From equation (2.80) it follows that $H(p)$ is periodic in $p$. Subsequently it is inferred from equations (2.78) and (2.79) that $U(p)$ and $V(p)$ are also periodic functions of $p$. From equations (2.74) and (2.77) it follows that $H, U$ and $V$ are oscillating in phase, i.e. they reach their extremal values simultaneously. 
The four parameters $\alpha, \beta, \gamma$ and $\sigma$ are replaced by quantities of physical interest by putting

$$
\begin{array}{ll}
H(p)=\bar{H}_{0}+\bar{A} \Phi(p), & \bar{H}_{0}=\frac{1}{2}\left(H_{\max }+H_{\min }\right), \\
U(p)=\bar{U}_{0}+\bar{B} \Psi(p), & \bar{U}_{0}=\frac{1}{2}\left(U_{\max }+U_{\min }\right), \\
V(p)=\bar{V}_{0}+\bar{C} \Omega(p), & \bar{V}_{0}=\frac{1}{2}\left(V_{\max }+V_{\min }\right),
\end{array}
$$

with $\Phi(p), \Psi(p)$ and $\Omega(p)$ periodic functions of $p$ oscillating between -1 and +1 . Using the fact that $\Phi(p), \Psi(p)$ and $\Omega(p)$ attain the value -1 or +1 simultaneously and also that $\Phi^{\prime}(p), \Psi^{\prime}(p)$ and $\Omega^{\prime}(p)$ vanish at the same points, equations (2.78), (2.79) and (2.80) lead to a set of six algebraical equations:

$$
\begin{aligned}
& \sigma=\kappa_{2}\left(\bar{U}_{0}+\bar{B}\right)-\kappa_{1}\left(\bar{V}_{0}+\bar{C}\right)=\kappa_{2}\left(\bar{U}_{0}-\bar{B}\right)-\kappa_{1}\left(\bar{V}_{0}-\bar{C}\right), \\
& \beta=-\omega\left(\bar{H}_{0}+\bar{A}\right)+\kappa_{1}\left(\bar{U}_{0}+\bar{B}\right)\left(\bar{H}_{0}+\bar{A}\right)+\kappa_{2}\left(\bar{V}_{0}+\bar{C}\right)\left(\bar{H}_{0}+\bar{A}\right)= \\
& =-\omega\left(\bar{H}_{0}-\bar{A}\right)+\kappa_{1}\left(\bar{U}_{0}-\bar{B}\right)\left(\bar{H}_{0}-\bar{A}\right)+\kappa_{2}\left(\bar{V}_{0}-\bar{C}\right)\left(\bar{H}_{0}-\bar{A}\right), \\
& \alpha\left(\bar{H}_{0}+\bar{A}\right)+\gamma+\frac{\beta^{2}}{\left(\kappa_{1}^{2}+\kappa_{2}^{2}\right)\left(\bar{H}_{0}+\bar{A}\right)}-\left(\bar{H}_{0}+\bar{A}\right)^{2}=0, \\
& \alpha\left(\bar{H}_{0}-\bar{A}\right)+\gamma+\frac{\beta^{2}}{\left(\kappa_{1}^{2}+\kappa_{2}^{2}\right)\left(\bar{H}_{0}-\bar{A}\right)}-\left(\bar{H}_{0}-\bar{A}\right)^{2}=0 .
\end{aligned}
$$

Using these equations in order to eliminate the constants $\alpha, \beta, \gamma$ and $\sigma$ we get the differential equation for $\Phi(p)$ :

$$
\mu \omega^{2} \Phi_{p}^{2}=\left(1-\Phi^{2}\right)\left[1-\frac{\left(\bar{H}_{0}^{2}-\bar{A}^{2}\right)\left(\omega-\kappa_{1} \bar{U}_{0}-\kappa_{2} \bar{V}_{0}\right)^{2}}{\left(\kappa_{1}^{2}+\kappa_{2}^{2}\right) \bar{H}_{0}^{2}\left(\bar{H}_{0}+\bar{A} \Phi\right)}\right]
$$

and the relations

$$
\begin{aligned}
& \frac{\bar{B}}{\kappa_{1}}=\frac{\bar{C}}{\kappa_{2}}=\frac{\bar{A}\left(\omega-\kappa_{1} \bar{U}_{0}-\kappa_{2} \bar{V}_{0}\right)}{\bar{H}_{0}\left(\kappa_{1}^{2}+\kappa_{2}^{2}\right)}, \\
& \sigma=\kappa_{2} \bar{U}_{0}-\kappa_{1} \bar{V}_{0} .
\end{aligned}
$$

Introducing the constants $A, B, C, \eta_{1}, u_{1}$ and $v_{1}$, all of order of magnitude one, by means of 


$$
\begin{aligned}
& \bar{A}=\varepsilon A, \quad \bar{B}=\varepsilon B, \quad \bar{C}=\varepsilon C, \\
& \bar{H}_{0}=1+\varepsilon^{2} \eta_{1}, \quad \bar{U}_{0}=\varepsilon^{2} u_{1}, \quad \bar{V}_{0}=\varepsilon^{2} v_{1},
\end{aligned}
$$

and expanding the fraction in equation (2.81) in powers of $\varepsilon$ we arrive at the differential equation:

$$
\begin{aligned}
& \mu \omega^{2}\left(\kappa_{1}^{2}+\kappa_{2}^{2}\right) \Phi_{p}^{2}=\left(1-\Phi^{2}\right)\left[\kappa_{1}^{2}+\kappa_{2}^{2}-\omega^{2}+\varepsilon A \omega^{2} \Phi+\right. \\
& \left.+\varepsilon^{2}\left\{A^{2} \omega^{2}+2 \omega\left(\kappa_{1} u_{1}+\kappa_{2} v_{1}\right)+\omega^{2} \eta_{1}-A^{2} \omega^{2} \Phi^{2}\right\}\right]+O\left(\varepsilon^{3}\right),
\end{aligned}
$$

which is the two-dimensional analogy of equation (2.43).

Application of Lindstedt's method leads to the following asymptotic series expansion for $H(p)$ :

$H(p)=1+\varepsilon A \cos 2 \pi p+\varepsilon^{2} \eta_{1}+\frac{\varepsilon^{2} A^{2}}{16 \pi^{2} \mu\left(\kappa_{1}^{2}+\kappa_{2}^{2}\right)}[\cos 4 \pi p-1]+O\left(\varepsilon^{3}\right)$.

The dispersion relation is found to be

$$
\begin{aligned}
& \kappa_{1}^{2}+\kappa_{2}^{2}-\omega^{2}=4 \pi^{2} \mu \omega^{2}\left(\kappa_{1}^{2}+\kappa_{2}^{2}\right)+\varepsilon^{2}\left[\frac{3 A^{2} \omega^{2}}{32 \pi^{2} \mu\left(\kappa_{1}^{2}+\kappa_{2}^{2}\right)}+\right. \\
& \left.-2 \omega\left(\kappa_{1} u_{1}+\kappa_{2} v_{1}\right)-\frac{1}{2} A^{2} \omega^{2}-\omega^{2} \eta_{1}\right]+O\left(\varepsilon^{3}\right)
\end{aligned}
$$

and the corresponding asymptotic expansions of $U(p)$ and $V(p)$ become:

$$
\begin{aligned}
U(p) & =\frac{\varepsilon A \omega \kappa_{1}}{\kappa_{1}^{2}+\kappa_{2}^{2}} \cos 2 \pi p+\varepsilon^{2}\left[u_{1}+\right. \\
& \left.+\left\{\frac{A^{2} \omega \kappa_{1}}{2\left(\kappa_{1}^{2}+\kappa_{2}^{2}\right)}-\frac{A^{2} \omega \kappa_{1}}{16 \pi^{2} \mu\left(\kappa_{1}^{2}+\kappa_{2}^{2}\right)^{2}}\right\}\{1-\cos 4 \pi p\}\right]+O\left(\varepsilon^{3}\right), \\
V(p) & =\frac{\varepsilon A \omega \kappa_{2}}{\kappa_{1}^{2}+\kappa_{2}^{2}} \cos 2 \pi p+\varepsilon^{2}\left[v_{1}+\right. \\
& \left.+\left\{\frac{A^{2} \omega \kappa_{2}}{2\left(\kappa_{1}^{2}+\kappa_{2}^{2}\right)}-\frac{A^{2} \omega \kappa_{2}}{16 \pi^{2} \mu\left(\kappa_{1}^{2}+\kappa_{2}^{2}\right)^{2}}\right\}\{1-\cos 4 \pi p\}\right]+O\left(\varepsilon^{3}\right) .
\end{aligned}
$$

Introducing the mean waveheight $H_{0}$ and the mean velocities $U_{0}$ and $V_{0}$ : 


$$
\begin{aligned}
& H_{0}=\varepsilon^{2}\left[\eta_{1}-\frac{A^{2}}{16 \pi^{2} \mu\left(\kappa_{1}^{2}+\kappa_{2}^{2}\right)}\right], \\
& U_{0}=\varepsilon^{2}\left[u_{1}+\frac{A^{2} \omega \kappa_{1}}{2\left(\kappa_{1}^{2}+\kappa_{2}^{2}\right)}-\frac{A^{2} \omega \kappa_{1}}{16 \pi^{2} \mu\left(\kappa_{1}^{2}+\kappa_{1}^{2}\right)^{2}}\right], \\
& V_{0}=\varepsilon^{2}\left[v_{1}+\frac{A^{2} \omega \kappa_{2}}{2\left(\kappa_{1}^{2}+\kappa_{2}^{2}\right)}-\frac{A^{2} \omega \kappa_{2}}{16 \pi^{2} \mu\left(\kappa_{1}^{2}+\kappa_{2}^{2}\right)^{2}}\right],
\end{aligned}
$$

we obtain the final asymptotic expansions for $H(p), U(p)$ and $V(p)$ :

$$
\begin{aligned}
H(p) & =1+H_{0}+\varepsilon A \cos 2 \pi p+\frac{\varepsilon^{2} A^{2}}{16 \pi^{2} \mu\left(\kappa_{1}^{2}+\kappa_{2}^{2}\right)} \cos 4 \pi p+O\left(\varepsilon^{3}\right), \\
U(p) & =U_{0}+\frac{\varepsilon A \omega \kappa_{1}}{\kappa_{1}^{2}+\kappa_{2}^{2}} \cos 2 \pi p+ \\
& +\varepsilon^{2}\left[\frac{A^{2} \omega \kappa_{1}}{16 \pi^{2} \mu\left(\kappa_{1}^{2}+\kappa_{2}^{2}\right)^{2}}-\frac{A^{2} \omega \kappa_{1}}{2\left(\kappa_{1}^{2}+\kappa_{2}^{2}\right)}\right] \cos 4 \pi p+O\left(\varepsilon^{3}\right), \\
V(p) & =V_{0}+\frac{\varepsilon A \omega \kappa_{2}}{\kappa_{1}^{2}+\kappa_{2}^{2}} \cos 2 \pi p+ \\
& +\varepsilon^{2}\left[\frac{A^{2} \omega \kappa_{2}}{16 \pi^{2} \mu\left(\kappa_{1}^{2}+\kappa_{2}^{2}\right)^{2}}-\frac{A^{2} \omega \kappa_{2}}{2\left(\kappa_{1}^{2}+\kappa_{2}^{2}\right)}\right] \cos 4 \pi p+O\left(\varepsilon^{3}\right),
\end{aligned}
$$

The dispersion relation transforms into

$$
\begin{aligned}
& \kappa_{1}^{2}+\kappa_{2}^{2}-\omega^{2}=4 \pi^{2} \mu \omega^{2}\left(\kappa_{1}^{2}+\kappa_{2}^{2}\right)-\omega^{2} H_{0}-2 \omega\left(\kappa_{1} U_{0}+\kappa_{2} V_{0}\right)+ \\
& +\varepsilon^{2}\left[\frac{1}{2} A^{2} \omega^{2}-\frac{3 A^{2} \omega^{2}}{32 \pi^{2} \mu\left(\kappa_{1}^{2}+\kappa_{2}^{2}\right)}\right]+O\left(\varepsilon^{4}\right) .
\end{aligned}
$$

To the same order of approximation dispersion relation (2.90) is equivalent with

where $E=\varepsilon^{2} A^{2}$ and

$$
\begin{aligned}
\left(\kappa_{1}^{2}+\kappa_{2}^{2}\right)\left(\omega-\omega_{0}\right) & =\omega_{0}^{2}\left(\kappa_{1} U_{0}+\kappa_{2} V_{0}\right)+\frac{1}{2} H_{0} \omega_{0}^{3}+ \\
& -\frac{1}{4} E \omega_{0}^{3}\left[1-\frac{3}{16 \pi^{2} \mu\left(\kappa_{1}^{2}+\kappa_{2}^{2}\right)}\right],
\end{aligned}
$$

$$
\omega_{0}\left(\kappa_{1}, \kappa_{2}\right)=\left\{\frac{\kappa_{1}^{2}+\kappa_{2}^{2}}{1+4 \pi^{2} \mu\left(\kappa_{1}^{2}+\kappa_{2}^{2}\right)}\right\}^{\frac{1}{2}} .
$$




\section{II.9 Conservative equations and integral relations for two-dimensional Boussinesq} waves

A slowly varying wavetrain solution of the two-dimensional Boussinesq equations (2.70), (2.71) and (2.72) is considered to be approximately of the form

$$
h=H(p, x, y, t), \quad u=U(p, x, y, t), \quad v=V(p, x, y, t),
$$

where the dependence of $H, U$ and $V$ on $p$ is the same as for the uniform wavetrain solution of section II. 8 but now $p$ stands for a general phase function

$$
p=K S(x, y, t) .
$$

The wavenumbers $\kappa_{1}, \kappa_{2}$ and the frequency $\omega$ are defined as

$$
\kappa_{1}(x, y, t)=\frac{\partial S}{\partial x}, \quad \kappa_{2}(x, y, t)=\frac{\partial S}{\partial y}, \quad \omega(x, y, t)=-\frac{\partial S}{\partial t},
$$

leading to the relations

$$
\frac{\partial \omega}{\partial x}+\frac{\partial \kappa_{1}}{\partial t}=0, \quad \frac{\partial \omega}{\partial y}+\frac{\partial \kappa_{2}}{\partial t}=0, \quad \frac{\partial \kappa_{1}}{\partial y}=\frac{\partial \kappa_{2}}{\partial x},
$$

of which only two equations are independent.

The slow dependence of $H, U$ and $V$ is introduced by considering the parameters $A, B, C, H_{0}, U_{0}$ and $V_{0}$ (and likewise $\kappa_{1}, \kappa_{2}$ and $\omega$ ) as functions of $x, y$ and $t$. The final asymptotic expansion of the uniform wavetrain solution as given by equations (2.87), (2.88), (2.89) and dispersion relation (2.91) contains only the parameters $A, H_{0}, U_{0}, V_{0}, \kappa_{1}, \kappa_{2}$ and $\omega$ whereas $B$ and $C$ may be found from them by using the two relations (2.82). Dispersion relation (2.91) and relations (2.95) constitute three equations connecting the seven unknown quantities $A, H_{0}, U_{0}, V_{0}, \kappa_{1}, \kappa_{2}$ and $\omega$. The four remaining equations are obtained now by an averaging of conservation laws.

The governing equations (2.70), (2.71) and (2.72) may be written in conservative form by using relation (2.73):

$$
\begin{aligned}
& \frac{\partial}{\partial t}(h)+\frac{\partial}{\partial x}(u h)+\frac{\partial}{\partial y}(v h)=0, \\
& \frac{\partial}{\partial t}(u)+\frac{\partial}{\partial x}\left[\frac{1}{2}\left(u^{2}+v^{2}\right)+h+\frac{\mu}{K^{2}} h_{t t}\right]=0, \\
& \frac{\partial}{\partial t}(v)+\frac{\partial}{\partial y}\left[\frac{1}{2}\left(u^{2}+v^{2}\right)+h+\frac{\mu}{K^{2}} h_{t t}\right]=0 .
\end{aligned}
$$


Substitution of equations (2.93) into these conservation laws yields terms of order $K$ which are equal to zero because of equations (2.74), (2.75) and (2.76). The remaining parts are of order unity and by averaging over one period in $p$ we find from equation (2.96):

$$
\int_{0}^{1} F_{1}(p, x, y, t) d p=0,
$$

with abbreviation

$$
F_{1}(p, x, y, t)=H_{t}+(U H)_{x}+(V H)_{y} .
$$

Conservative equation (2.97) leads to

where

$$
\int_{0}^{1} F_{2}(p, x, y, t) d p+O\left(\frac{1}{K}\right)=0,
$$

$$
\begin{aligned}
& F_{2}(p, x, y, t)=U_{t}+\frac{1}{2}\left(U^{2}+V^{2}\right)_{x}+H_{x}+\mu \omega^{2} H_{p p x}+ \\
& +2 \mu \omega \omega_{x} H_{p p}-\mu \kappa_{1} \omega_{t} H_{p p}-2 \mu \kappa_{1} \omega H_{p p t},
\end{aligned}
$$

and conservative equation (2.98) gives

where

$$
\int_{0}^{1} F_{3}(p, x, y, t) d p+O\left(\frac{1}{K}\right)=0,
$$

$$
\begin{aligned}
& F_{3}(p, x, y, t)=V_{t}+\frac{1}{2}\left(U^{2}+V^{2}\right)_{y}+H_{y}+\mu \omega^{2} H_{p p y}+ \\
& +2 \mu \omega \omega_{y} H_{p p}-\mu \kappa_{2} \omega_{t} H_{p p}-2 \mu \kappa_{2} \omega H_{p p t} .
\end{aligned}
$$

Further conservation laws may be found by multiplication of equation (2.96) by $u$ and adding the result to equation (2.97) multiplied by $h$, viz.:

$\frac{\partial}{\partial t}\left[u h-\frac{\mu}{K^{2}} h_{x} h_{t}\right]+\frac{\partial}{\partial x}\left[u^{2} h+\frac{1}{2} h^{2}+\frac{\mu}{K^{2}} h h_{t t}+\frac{\mu}{2 K^{2}} h_{t}^{2}\right]+\frac{\partial}{\partial y}[u v h]=0$,

and by multiplication of equation (2.96) by $v$ and adding the result to equation (2.98) multiplied by $h$, viz. :

$\frac{\partial}{\partial t}\left[v h-\frac{\mu}{K^{2}} h_{y} h_{t}\right]+\frac{\partial}{\partial x}[u v h]+\frac{\partial}{\partial y}\left[v^{2} h+\frac{1}{2} h^{2}+\frac{\mu}{K^{2}} h h_{t t}+\frac{\mu}{2 K^{2}} h_{t}^{2}\right]=0$. 
Application of the averaging technique to conservative equations (2.102) and (2.103) leads to the following integral relations repectively:

$$
\begin{aligned}
& \int_{0}^{1}\left(U F_{1}+H F_{2}\right) d p+O\left(\frac{1}{K}\right)=0 \\
& \int_{0}^{1}\left(V F_{1}+H F_{3}\right) d p+O\left(\frac{1}{K}\right)=0 .
\end{aligned}
$$

Substitution of the asymptotic expansions of $H(p, x, y, t), U(p, x, y, t)$ and $V(p, x, y, t)$ into integral relations (2.99), (2.100) and (2.101) gives successively

$$
\begin{aligned}
& \frac{\partial H_{0}}{\partial t}+\frac{\partial}{\partial x}\left[U_{0}+\frac{E \omega_{0} \kappa_{1}}{2\left(\kappa_{1}^{2}+\kappa_{2}^{2}\right)}\right]+\frac{\partial}{\partial y}\left[V_{0}+\frac{E \omega_{0} \kappa_{2}}{2\left(\kappa_{1}^{2}+\kappa_{2}^{2}\right)}\right]+O\left(\varepsilon^{4}\right)=0, \\
& \frac{\partial U_{0}}{\partial t}+\frac{\partial}{\partial x}\left[H_{0}+\frac{E \omega_{0}^{2}}{4\left(\kappa_{1}^{2}+\kappa_{2}^{2}\right)}\right]+O\left(\varepsilon^{4}\right)=0, \\
& \frac{\partial V_{0}}{\partial t}+\frac{\partial}{\partial y}\left[H_{0}+\frac{E \omega_{0}^{2}}{4\left(\kappa_{1}^{2}+\kappa_{2}^{2}\right)}\right]+O\left(\varepsilon^{4}\right)=0 .
\end{aligned}
$$

The first of these equations may be considered as an averaged continuity equation, the second and third equation as averaged momentum equations in the $x$ - and $y$ direction respectively.

Integral relations (2.104) and (2.105) lead to the equations:

$$
\begin{aligned}
& \frac{\partial}{\partial t}\left[U_{0}+\frac{E \omega \kappa_{1}}{2\left(\kappa_{1}^{2}+\kappa_{2}^{2}\right)}\right]+\frac{\partial}{\partial x}\left[\frac{1}{4} E+H_{0}+\frac{E \omega^{2} \kappa_{1}^{2}}{2\left(\kappa_{1}^{2}+\kappa_{2}^{2}\right)^{2}}\right]+ \\
& +\frac{\partial}{\partial y}\left[\frac{E \omega^{2} \kappa_{1} \kappa_{2}}{2\left(\kappa_{1}^{2}+\kappa_{2}^{2}\right)^{2}}\right]+2 \pi^{2} \mu\left[E \kappa_{1} \omega_{t}+E_{t} \kappa_{1} \omega-\frac{1}{2} \omega^{2} E_{x}+\right. \\
& \left.-2 \omega \omega_{x} E\right]+O\left(\varepsilon^{4}\right)=0, \\
& \frac{\partial}{\partial t}\left[V_{0}+\frac{E \omega \kappa_{2}}{2\left(\kappa_{1}^{2}+\kappa_{2}^{2}\right)}\right]+\frac{\partial}{\partial x}\left[\frac{E \omega^{2} \kappa_{1} \kappa_{2}}{2\left(\kappa_{1}^{2}+\kappa_{2}^{2}\right)^{2}}\right]+ \\
& +\frac{\partial}{\partial y}\left[\frac{1}{4} E+H_{0}+\frac{E \omega^{2} \kappa_{2}^{2}}{2\left(\kappa_{1}^{2}+\kappa_{2}^{2}\right)^{2}}\right]+2 \pi^{2} \mu\left[E \kappa_{2} \omega_{t}+\right. \\
& \left.+E_{t} \kappa_{2} \omega-\frac{1}{2} \omega^{2} E_{y}-2 \omega \omega_{y} E\right]+O\left(\varepsilon^{4}\right)=0 .
\end{aligned}
$$


Making use of the fact that the coefficient of $2 \pi^{2} \mu$ in equation (2.109) is equal to

$$
\frac{\partial}{\partial t}\left(E \omega \kappa_{1}\right)-\frac{\partial}{\partial x}\left(\frac{1}{2} E \omega^{2}\right)
$$

and using equation (2.107) in order to eliminate $U_{0}$ and $H_{0}$, equation (2.109) is found to reduce to

$$
\frac{\partial}{\partial t}\left(\frac{\kappa_{1} E}{2 \omega_{0}}\right)+\frac{\partial}{\partial x}\left(C_{01} \frac{\kappa_{1} E}{2 \omega_{0}}\right)+\frac{\partial}{\partial y}\left(C_{02} \frac{\kappa_{1} E}{2 \omega_{0}}\right)+O\left(\varepsilon^{4}\right)=0,
$$

where the $x$ - and $y$-components $C_{01}$ and $C_{02}$ of the linear group velocity vector are defined by

$$
\begin{aligned}
& C_{01}=\frac{\partial \omega_{0}}{\partial \kappa_{1}}=\frac{\kappa_{1} \omega_{0}^{3}}{\left(\kappa_{1}^{2}+\kappa_{2}^{2}\right)^{2}}, \\
& C_{02}=\frac{\partial \omega_{0}}{\partial \kappa_{2}}=\frac{\kappa_{2} \omega_{0}^{3}}{\left(\kappa_{1}^{2}+\kappa_{2}^{2}\right)^{2}} .
\end{aligned}
$$

Similarly it can be shown that equation (2.110) reduces to

$$
\frac{\partial}{\partial t}\left(\frac{\kappa_{2} E}{2 \omega_{0}}\right)+\frac{\partial}{\partial x}\left(C_{01} \frac{\kappa_{2} E}{2 \omega_{0}}\right)+\frac{\partial}{\partial y}\left(C_{02} \frac{\kappa_{2} E}{2 \omega_{0}}\right)+O\left(\varepsilon^{4}\right)=0 .
$$

By virtue of the relation

$$
\begin{aligned}
& \frac{\partial}{\partial t}\left\{f\left(\kappa_{1}, \kappa_{2}\right)\right\}+C_{01} \frac{\partial}{\partial x}\left\{f\left(\kappa_{1}, \kappa_{2}\right)\right\}+C_{02} \frac{\partial}{\partial y}\left\{f\left(\kappa_{1}, \kappa_{2}\right)\right\}= \\
& =\frac{\partial f}{\partial \kappa_{1}} \frac{\partial}{\partial x}\left\{\omega-\omega_{0}\left(\kappa_{1}, \kappa_{2}\right)\right\}+\frac{\partial f}{\partial \kappa_{2}} \frac{\partial}{\partial y}\left\{\omega-\omega_{0}\left(\kappa_{1}, \kappa_{2}\right)\right\}=O\left(\varepsilon^{2}\right),
\end{aligned}
$$

holding for any function $f\left(\kappa_{1}, \kappa_{2}\right)$ of $\kappa_{1}$ and $\kappa_{2}$, both equation (2.111) and (2.112) are asymptotically equivalent to the averaged energy equation:

$$
\frac{\partial E}{\partial t}+\frac{\partial}{\partial x}\left(C_{01} E\right)+\frac{\partial}{\partial y}\left(C_{02} E\right)=0
$$

Hence we have a set of seven equations for the determination of the seven slowly varying functions $\omega(x, y, t), \kappa_{1}(x, y, t), \kappa_{2}(x, y, t), E(x, y, t), U_{0}(x, y, t), V_{0}(x, y, t)$ and $H_{0}(x, y, t)$, viz. relations (2.95), equations (2.106), (2.107), (2.108) and (2.113) together with dispersion relation (2.91). 
III.1 Formulation of the problem and the asymptotic representation of a slowly varying wavetrain

The general formulation of the boundary value problem for the velocity potential of two-dimensional irrotational surface waves of finite amplitude on water of finite depth (Stokes waves) is given in the Appendix by equation (A.1) and boundary conditions (A.2), (A.3) and (A.4). After stretching the horizontal coordinate and the time by a large factor $K$ and leaving the vertical coordinate unaltered the boundary value problem for the potential $\tilde{\Phi}(x, y, t)$, where $x$ and $t$ now denote the stretched variables, becomes as follows: within the fluid bounded by the bottom $y=-h_{0}$ and the free surface $y=\eta(x, t)$ the velocity potential $\tilde{\Phi}(x, y, t)$ satisfies the partial differential equation:

$$
\widetilde{\Phi}_{x x}+K^{2} \widetilde{\Phi}_{y y}=0
$$

with boundary conditions

$$
\begin{aligned}
& {\left[\tilde{\Phi}_{y}\right]_{y=-h_{0}}=0,} \\
& K\left[\tilde{\Phi}_{t}\right]_{y=\eta(x, t)}+\frac{1}{2}\left[\tilde{\Phi}_{x}^{2}+K^{2} \tilde{\Phi}_{y}^{2}\right]_{y=\eta(x, t)}+g K^{2} \eta=0, \\
& K \eta_{t}+\eta_{x}\left[\tilde{\Phi}_{x}\right]_{y=\eta(x, t)}-K^{2}\left[\tilde{\Phi}_{y}\right]_{y=\eta(x, t)}=0,
\end{aligned}
$$

where the Bernoulli constant in boundary condition (3.3) has been taken equal to zero by absorbing it into the potential $\tilde{\Phi}(x, y, t)$.

In order to consider slowly varying wavetrains with $K^{-1}$ as a measure for the slow variations of amplitude, wavenumber, frequency, etc. we have to expand both the potential $\tilde{\Phi}(x, y, t)$ and the free surface elevation $\eta(x, t)$ in asymptotic series in $K$. At first we notice that the derivatives of $\tilde{\Phi}(x, y, t)$ necessarily should be bounded for all values of $x, y$ and $t$. This means that $\widetilde{\Phi}(x, y, t)$ itself may contain terms that become unbounded for large values of $x$ and $t$. For instance consider the uniform wavetrain solution studied in the Appendix: its first term $K(\beta x-\gamma t)$ accounting for the mean velocity $\beta$ and the Bernoulli constant $\gamma$ is not bounded for large $x$ and $t$.

Furthermore the phase function $S$ of the problem is taken as a function of $x$ and $t$ only: rapid variations only occur in the $x$ - and $t$-directions and not in the direction of the vertical coordinate $y$. Hence for a slowly varying wavetrain we introduce the following asymptotic representation: 


$$
\begin{aligned}
\widetilde{\Phi}(x, y, t) & =K \Psi(x, t)+\Phi[K S(x, t), x, y, t]+\Psi_{1}(x, t)+ \\
& +\frac{1}{K} \chi[K S(x, t), x, y, t]+\frac{1}{K} \Psi_{2}(x, t)+O\left(\frac{1}{K^{2}}\right), \\
\eta(x, t) & =U[K S(x, t), x, t]+\frac{1}{K} V[K S(x, t), x, t]+O\left(\frac{1}{K^{2}}\right),
\end{aligned}
$$

with $\Phi, \chi, U$ and $V$ bounded functions for all values of $x, y$ and $t$. The functions $\Psi$, $\Psi_{1}$ and $\Psi_{2}$ account for the unbounded parts of the potential $\tilde{\Phi}$ and also for the absorption of the Bernoulli constant into $\tilde{\Phi}$. However, the derivatives of $\Psi, \Psi_{1}$ and $\Psi_{2}$ should be bounded for all $x$ and $t$.

Introducing the notations

$$
\begin{array}{ll}
\kappa(x, t)=S_{x}, & \omega(x, t)=-S_{t}, \quad p=K S(x, t), \\
\beta(x, t)=\Psi_{x}, & \gamma(x, t)=-\Psi_{t}, \\
\beta_{1}(x, t)=\Psi_{1 x}, & \gamma_{1}(x, t)=-\Psi_{1 t}, \\
\beta_{2}(x, t)=\Psi_{2 x}, & \gamma_{2}(x, t)=-\Psi_{2 t},
\end{array}
$$

we insert asymptotic expansions (3.5) and (3.6) into boundary value problem (3.1), (3.2), (3.3) and (3.4) for $\widetilde{\Phi}(x, y, t)$. The highest power of $K$ leads to a boundary value problem involving $\Phi(p, x, y, t), U(p, x, t)$ and $\Psi(x, t)$. The leading term $\Phi(p, x, y, t)$ satisfies the equation

$$
\kappa^{2} \Phi_{p p}+\Phi_{y y}=0, \quad \text { for } \quad-h_{0} \leqq y \leqq U(p, x, t),
$$

with boundary condition at the bottom $y=-h_{0}$ :

$$
\Phi_{y}=0
$$

and free surface conditions to be satisfied on $y=U(p, x, t)$ :

$$
\begin{aligned}
& g U+(\beta \kappa-\omega) \Phi_{p}+\frac{1}{2}\left(\Phi_{y}^{2}+\kappa^{2} \Phi_{p}^{2}\right)=\gamma-\frac{1}{2} \beta^{2}, \\
& (\beta \kappa-\omega) U_{p}+\kappa^{2} U_{p} \Phi_{p}-\Phi_{y}=0 .
\end{aligned}
$$

The next power of $K$ yields a boundary value problem containing the second terms $\chi(p, x, y, t), V(p, x, t)$ and $\Psi_{1}(x, t)$. The equation is

$$
\kappa^{2} \chi_{p p}+\chi_{y y}=-\kappa_{x} \Phi_{p}-2 \kappa \Phi_{p x}-\beta_{x}, \quad\left(-h_{0} \leqq y \leqq U(p, x, t)\right)
$$


with the boundary condition at the bottom $y=-h_{0}$ :

$$
\chi_{y}=0
$$

and free surface conditions on $y=U(p, x, t)$ :

$$
\begin{aligned}
& {\left[-\omega \Phi_{p y}+\left(\beta+\kappa \Phi_{p}\right) \kappa \Phi_{p y}+\Phi_{y} \Phi_{y y}+g\right] V+} \\
& +\left[-\omega+\kappa\left(\beta+\kappa \Phi_{p}\right)\right] \chi_{p}+\Phi_{y} \chi_{y}+\beta \beta_{1}-\gamma_{1}= \\
& =-\Phi_{t}-\left(\beta+\kappa \Phi_{p}\right) \Phi_{x} \\
& {\left[-\omega+\kappa\left(\beta+\kappa \Phi_{p}\right)\right] V_{p}+\kappa^{2} U_{p} \chi_{p}-\chi_{y}+} \\
& +\left[\kappa^{2} U_{p} \Phi_{p y}-\Phi_{y y}\right] V+\beta_{1} \kappa U_{p}= \\
& =-U_{t}-\kappa U_{p} \Phi_{x}-\left(\beta+\kappa \Phi_{p}\right) U_{x} .
\end{aligned}
$$

Notice that the free surface conditions of these problems are imposed on the curve $y=U(p, x, t)$.

The first boundary value problem involving $\Phi, U$ and $\Psi$ is seen to be exactly equivalent to the problem for the uniform wavetrain solution given in the Appendix by equation (A.6) and boundary conditions (A.7), (A.8) and (A.9) with $\kappa \theta$ replaced by $p$. The solution is given in terms of two asymptotic power series in $\varepsilon$ for $\Phi$ and $U$. The small parameter is $\varepsilon=\kappa a$ where $a$ is a measure for the amplitude of the wave. Quoting the results from the Appendix we have up to terms of order $\varepsilon^{2}$ :

$$
\begin{aligned}
\Phi(p, x, y, t)= & A_{1} \cosh \left[\kappa\left(y+h_{0}\right)\right] \sin p+ \\
& +A_{2} \cosh \left[2 \kappa\left(y+h_{0}\right)\right] \sin 2 p+O\left(\varepsilon^{3}\right), \\
U(p, x, t)= & b+a \cos p+a_{2} \cos 2 p+O\left(\varepsilon^{3}\right),
\end{aligned}
$$

with $A_{1}=O(\varepsilon), A_{2}=O\left(\varepsilon^{2}\right)$ and $a_{2}=O\left(\varepsilon^{2}\right)$ defined by equation (A.26), (A.27) and (A. 28) of the Appendix.

The leading terms $\Phi(p, x, y, t), U(p, x, t)$ and $\Psi(x, t)$ of the asymptotic representation of the slowly varying wavetrain represent locally a uniform wavetrain containing six slowly varying parameters, viz. the wavenumber $\kappa(x, t)$ and frequency $\omega(x, t)$ being of order unity, the amplitude $a(x, t)$ being of order $\varepsilon$, and the mean velocity $\beta(x, t)$, the mean waveheight $b(x, t)$ and the pseudo-frequency $\gamma(x, t)$ which are quantities of order $\varepsilon^{2}$. We have already four equations relating these six functions, viz. dispersion relation (A.29): 


$$
\omega=\omega_{0}(\kappa)+\beta \kappa+\frac{g b \kappa^{2}}{2 \omega_{0} \cosh ^{2} \kappa h_{0}}+\frac{1}{2} g \kappa^{3} a^{2} \frac{D_{0}}{\omega_{0}},
$$

where $\omega_{0}^{2}(\kappa)=g \kappa \operatorname{tgh} \kappa h_{0}$ and

$$
D_{0}=\frac{9 \operatorname{tgh}^{4} \kappa h_{0}-10 \operatorname{tgh}^{2} \kappa h_{0}+9}{8 \operatorname{tgh}^{3} \kappa h_{0}},
$$

the pseudo-dispersion relation:

$$
g b=\gamma-\frac{g \kappa a^{2}}{2 \sinh 2 \kappa h_{0}},
$$

and the relations

$$
\begin{aligned}
& \frac{\partial \kappa}{\partial t}+\frac{\partial \omega}{\partial x}=0 \\
& \frac{\partial \beta}{\partial t}+\frac{\partial \gamma}{\partial x}=0
\end{aligned}
$$

following from the definition of $\kappa, \omega, \beta$ and $\gamma$.

The two remaining equations are obtained in the next section by imposing conditions of boundedness on the second terms $\chi(p, x, y, t)$ and $V(p, x, t)$ of the asymptotic representations (3.5) and (3.6) for slowly varying wavetrains.

III.2 Investigation of the boundary value problem for $\chi(p, x, y, t), V(p, x, t)$ and $\psi_{1}(x, t)$

For Stokes waves it does not seem to be possible to derive integral relations analogous to those obtained in Chapters I and II for shallow water waves. We proceed in the present case by expanding $\chi$ and $V$ in asymptotic series with respect to $\varepsilon$ and formulating the boundary value problems for the first order and second order terms of these series. In doing so we will meet secular terms containing $\Phi, U$ and $\Psi$ which have to vanish in order to ensure the boundedness of $\chi$ and $V$. This will provide the remaining equations relating the six unknown functions $\kappa, \omega, a, \gamma, \beta, b$.

Thus we introduce the expansions

$$
\begin{aligned}
& \chi(p, x, y, t)=\chi_{1}(p, x, y, t)+\chi_{2}(p, x, y, t)+O\left(\varepsilon^{3}\right), \\
& V(p, x, t)=V_{1}(p, x, t)+V_{2}(p, x, t)+O\left(\varepsilon^{3}\right),
\end{aligned}
$$

where $\chi_{1}$ and $V_{1}$ are functions of order $\varepsilon$ and $\chi_{2}$ and $V_{2}$ are of order $\varepsilon^{2}$. 
We recall that $\Phi(p, x, y, t)$ and $U(p, x, t)$ are expanded as follows:

$$
\begin{aligned}
& \Phi(p, x, y, t)=\Phi_{1}(p, x, y, t)+\Phi_{2}(p, x, y, t)+O\left(\varepsilon^{3}\right), \\
& U(p, x, t)=U_{1}(p, x, t)+U_{2}(p, x, t)+O\left(\varepsilon^{3}\right),
\end{aligned}
$$

with $\Phi_{1}$ and $U_{1}$ of order $\varepsilon$ and $\Phi_{2}$ and $U_{2}$ of order $\varepsilon^{2}$.

Furthermore we notice that $\beta, \gamma, \beta_{1}$ and $\gamma_{1}$ are quantities of order of magnitude $\varepsilon^{2}$. Inserting these expansions into the boundary value problem for $\chi$ and $V$ we obtain for the terms of order $\varepsilon$ the following boundary value problem:

$$
\begin{aligned}
& \kappa^{2} \chi_{1 p p}+\chi_{1 y y}=-\kappa_{x} \Phi_{1 p}-2 \kappa \Phi_{1 p x} \text { for } \quad-h_{0} \leqq y \leqq 0 . \\
& \chi_{1 y}=0, \quad \text { on } y=-h_{0}, \\
& g V_{1}-\omega_{0} \chi_{1 p}=-\Phi_{1 t} \quad \text { on } \quad y=0 \\
& \omega_{0} V_{1 p}+\chi_{1 y}=U_{1 t} \quad \text { on } \quad y=0,
\end{aligned}
$$

Note that the expansion with respect to $\varepsilon$ implies that the free surface conditions are to be satisfied on $y=0$ and that $\chi_{1}$ and $V_{1}$ satisfy a linear inhomogeneous boundary value problem.

For $\chi_{2}$ and $V_{2}$ we obtain the linear boundary value problem:

$$
\begin{array}{lr}
\kappa^{2} \chi_{2 p p}+\chi_{2 y y}=-\kappa_{x} \Phi_{2 p}-2 \kappa \Phi_{2 p x}-\beta_{x}, & \text { for }-h_{0} \leqq y \leqq 0, \\
\chi_{2 y}=0, & \text { on } y=-h_{0}, \\
g V_{2}-\omega_{0} \chi_{2 p}=\omega_{0} V_{1} \Phi_{1 p y}+\omega_{0} U_{1} \chi_{1 p y}-\kappa^{2} \Phi_{1 p} \chi_{1 p}+\gamma_{1}+ \\
-\Phi_{1 y} \chi_{1 y}-\Phi_{2 t}-\kappa \Phi_{1 p} \Phi_{1 x}, & \text { on } y=0, \\
\omega_{0} V_{2 p}+\chi_{2 y}=\kappa^{2} \Phi_{1 p} V_{1 p}+\kappa^{2} U_{1 p} \chi_{1 p}-U_{1} \chi_{1 y y}-V_{1} \Phi_{1 y y}+ \\
+U_{2 t}+\kappa U_{1 p} \Phi_{1 x}+\kappa \Phi_{1 p} U_{1 x}, & \text { on } y=0 .
\end{array}
$$

At first we consider the boundary value problem for $\chi_{1}$ and $V_{1}$. Elimination of $V_{1}$ from free surface conditions (3.22) and (3.23) and using the explicit expressions for $\Phi_{1}$ and $U_{1}$ yields the free surface condition for $\chi_{1}$ :

$$
\frac{\omega_{0}^{2}}{g} \chi_{1 p p}+\chi_{1 y}=U_{1 t}+\frac{\omega_{0}}{g} \Phi_{1 p t}=\alpha_{0} \cos p, \quad \text { on } y=0
$$


with the abbreviation:

$$
\alpha_{0}=a_{t}+\frac{\omega_{0}}{g} \frac{\partial}{\partial t}\left(A_{1} \cosh \kappa h_{0}\right)=\frac{\omega_{0}}{a} \frac{\partial}{\partial t}\left(\frac{a^{2}}{\omega_{0}}\right)
$$

The differential equation for $\chi_{1}$ becomes:

$$
\begin{aligned}
& \kappa^{2} \chi_{1 p p}+\chi_{1 y y}=\alpha_{1} \cosh \left[\kappa\left(h_{0}+y\right)\right] \cos p+ \\
& +\alpha_{2}\left(h_{0}+y\right) \sinh \left[\kappa\left(h_{0}+y\right)\right] \cos p,
\end{aligned}
$$

where we have put

$$
\begin{aligned}
& \alpha_{1}=-\kappa_{x} A_{1}-2 \kappa A_{1 x}, \\
& \alpha_{2}=-2 \kappa \kappa_{x} A_{1},
\end{aligned}
$$

and where $A_{1}$ is defined by equation (A.26) of the Appendix.

A particular solution of equation (3.29) satisfying bottom condition (3.21) and which is bounded in the strip $-h_{0} \leqq y \leqq 0$, is given by the periodic function in $p$ :

$$
\begin{aligned}
\chi_{1}^{(P)} & =\frac{\alpha_{2}}{4 \kappa}\left(y+h_{0}\right)^{2} \cosh \left[\kappa\left(h_{0}+y\right)\right] \cos p+ \\
& +\left(\frac{\alpha_{1}}{2 \kappa}-\frac{\alpha_{2}}{4 \kappa^{2}}\right)\left(y+h_{0}\right) \sinh \left[\kappa\left(h_{0}+y\right)\right] \cos p .
\end{aligned}
$$

Introducing $\tilde{\chi}_{1}$ by putting:

$$
\chi_{1}=\chi_{1}^{(P)}+\tilde{\chi}_{1},
$$

then $\tilde{\chi}_{1}$ satisfies the equation

$$
\kappa^{2} \tilde{\chi}_{1 p p}+\tilde{\chi}_{1 y y}=0,
$$

with boundary conditions

and

$$
\tilde{\chi}_{1 y}=0, \quad \text { on } y=-h_{0},
$$

$$
\frac{\omega_{0}^{2}}{g} \tilde{\chi}_{1 p p}+\tilde{\chi}_{1 y}=\alpha \cos p \quad \text { on } y=0
$$

where we have put 


$$
\begin{aligned}
\alpha & =\alpha_{0}+\frac{\omega_{0}^{2}}{g}\left(\frac{\alpha_{1}}{2 \kappa}-\frac{\alpha_{2}}{4 \kappa^{2}}\right) h_{0} \sinh \kappa h_{0}+ \\
& -\frac{\alpha_{2}}{2 \kappa} h_{0} \cosh \kappa h_{0}-\left(\frac{\alpha_{1}}{2 \kappa}-\frac{\alpha_{2}}{4 \kappa^{2}}\right)\left(\sinh \kappa h_{0}+\kappa h_{0} \cosh \kappa h_{0}\right) .
\end{aligned}
$$

It may be verified that this boundary value problem for $\tilde{\chi}_{1}$ has a solution of the form:

$$
C \alpha\left\{p \sin p \cosh \left[\kappa\left(y+h_{0}\right)\right]-\kappa\left(y+h_{0}\right) \cos p \sinh \left[\kappa\left(y+h_{0}\right)\right]\right\},
$$

with $C$ a constant. This solution is not bounded for large $p$ and hence the term $\alpha \cos p$ in the free surface condition for $\tilde{\chi}_{1}$ must vanish. This leads to the equation:

$$
\begin{aligned}
\alpha & =\frac{\omega_{0}}{a} \frac{\partial}{\partial t}\left(\frac{a^{2}}{\omega_{0}}\right)-\kappa h_{0} A_{1 x} \frac{\sinh ^{2} \kappa h_{0}}{\cosh \kappa h_{0}}+A_{1} h_{0} \kappa_{x} \cosh \kappa h_{0}+ \\
& +A_{1 x}\left(\sinh \kappa h_{0}+\kappa h_{0} \cosh \kappa h_{0}\right)=0,
\end{aligned}
$$

which reduces after considerable manipulation to the energy density equation:

$$
\frac{\partial E}{\partial t}+\frac{\partial}{\partial x}\left[\omega_{0}^{\prime}(\kappa) E\right]=0, \quad E=\frac{a^{2}}{\omega_{0}} .
$$

For $\chi_{2}$ we have the equation

$$
\begin{aligned}
\kappa^{2} \chi_{2 p p}+\chi_{2 y y} & =-\beta_{x}-2 \kappa_{x} A_{2} \cosh \left[2 \kappa\left(y+h_{0}\right)\right] \cos 2 p+ \\
& -4 \kappa \frac{\partial}{\partial x}\left\{A_{2} \cosh \left[2 \kappa\left(y+h_{0}\right)\right]\right\} \cos 2 p .
\end{aligned}
$$

The inhomogeneous term of equation (3.31) contains terms with $\cos 2 p$ and a term $-\beta_{x}$ that is constant with respect to $p$. The terms with $\cos 2 p$ cause no difficulties but the constant term may induce unbounded terms in $p$ as will appear below.

Some calculation shows that the inhomogeneous terms in free surface conditions (3.26) and (3.27) are made up of terms with $\sin 2 p$ or $\cos 2 p$ and terms constant with respect to $p$. Eliminating $V_{2}$ from conditions (3.26) and (3.27) it is clear that only the constant terms in the righthand side of condition (3.27) will remain in the resulting free surface condition for $\chi_{2}$ because the constant terms of condition (3.26) vanish by differentiation with respect to $p$.

Writing condition (3.27) as

$$
\begin{aligned}
\omega_{0} V_{2 p}+\chi_{2 y} & =\kappa^{2} \frac{\partial}{\partial p}\left(V_{1} \Phi_{1 p}+U_{1} \chi_{1 p}\right)+U_{2 t}+\kappa U_{1 p} \Phi_{1 x}+ \\
& +\kappa \Phi_{1 p} U_{1 x}+U_{1}\left(\kappa_{x} \Phi_{1 p}+2 \kappa \Phi_{1 p x}\right), \quad \text { on } y=0,
\end{aligned}
$$


it is seen that the constant term in the righthand side is independent of $\chi_{1}$ and $V_{1}$. Schematically we find the following boundary value problem for $\chi_{2}$ :

$$
\begin{array}{lc}
\kappa^{2} \chi_{2 p p}+\chi_{2 y y}=-\beta_{x}+[\ldots] \cos 2 p, & \text { for }-h_{0} \leqq y \leqq 0, \\
\chi_{2 y}=0, & \text { on } y=-h_{0}, \\
\frac{\omega_{0}^{2}}{g} \chi_{2 p p}+\chi_{2 y}=b_{t}+\frac{1}{2} \kappa a\left(A_{1 x} \cosh \kappa h_{0}+A_{1} \kappa_{x} h_{0} \sinh \kappa h_{0}\right)+ \\
+\frac{1}{2} \frac{\partial}{\partial x}(a \kappa) \cdot A_{1} \cosh \kappa h_{0}+[\ldots] \cos 2 p, & \text { on } \quad y=0 .
\end{array}
$$

The terms with $\cos 2 p$ do not give rise to unbounded terms in $\chi_{2}$ and need not be considered further. Considering only the constant parts of the inhomogeneous terms and introducing a function $\tilde{\chi}_{2}$ by:

$$
\chi_{2}=-\frac{1}{2} \beta_{x}\left(y+h_{0}\right)^{2}+\tilde{\chi}_{2},
$$

the resulting boundary value problem for $\tilde{\chi}_{2}$ is similar to that for $\tilde{\chi}_{1}$, only with the term $\alpha \cos p$ in the free surface condition replaced by the constant term:

$$
\begin{aligned}
\sigma & =\beta_{x} h_{0}+b_{t}+\frac{1}{2} \kappa a\left(A_{1 x} \cosh \kappa h_{0}+A_{1} \kappa_{x} h_{0} \sinh \kappa h_{0}\right)+ \\
& +\frac{1}{2} \frac{\partial}{\partial x}(a \kappa) \cdot A_{1} \cosh \kappa h_{0} .
\end{aligned}
$$

Introducing the complex conjugate $\zeta(p, y)$ of $\tilde{\chi}_{2}(p, y)$ and putting

$$
f(z)=\tilde{\chi}_{2}(p, y)+i \zeta(p, y), \quad z=\frac{p}{\kappa}+i y,
$$

then $f(z)$ is an analytic function of $z$ that has to be bounded in the strip $-h_{0} \leqq I m$ $z \leqq 0$. The free surface condition for $\tilde{\chi}_{2}$ may be written as

$$
\frac{\omega_{0}^{2}}{g} \tilde{\chi}_{2 p p}+\tilde{\chi}_{2 y}=\frac{\omega_{0}^{2}}{g} \tilde{\chi}_{2 p p}-\kappa \zeta_{p}=\sigma, \quad \text { on } \quad y=0 .
$$

Integration with respect to $p$ transforms this condition into

$$
\frac{\omega_{0}^{2}}{g} \tilde{\chi}_{2 p}-\kappa \zeta=\sigma p+\text { constant, } \quad \text { on } \quad y=0 .
$$


Now both $\tilde{\chi}_{2 p}$ and $\zeta$ should be bounded for all $p$ on $y=0$, so $\sigma p$ is a secular term. Hence we have $\sigma=0$, which leads to the equation

$$
\frac{\partial b}{\partial t}+h_{0} \frac{\partial \beta}{\partial x}+\frac{\partial}{\partial x}\left(\frac{g a^{2} \kappa}{2 \omega_{0}}\right)=0
$$

This equation which may be considered as an averaged continuity equation, completes the set of six equations (3.16), (3.17), (3.18), (3.19), (3.30) and (3.33) for the determination of the six slowly varying functions $\kappa(x, t), \omega(x, t), a(x, t), \gamma(x, t), \beta(x, t)$ and $b(x, t)$. By elimination of $\omega(x, t$ and $\gamma(x, t)$ this set reduces to a set of four equations:

$$
\begin{aligned}
& \frac{\partial \kappa}{\partial t}+\frac{\partial}{\partial x}\left[\omega_{0}+\beta \kappa+\frac{g b \kappa^{2}}{2 \omega_{0} \cosh ^{2} \kappa h_{0}}+\frac{1}{2} g \kappa^{3} D_{0} E\right]=0, \\
& \frac{\partial E}{\partial t}+\frac{\partial}{\partial x}\left[\omega_{0}^{\prime}(\kappa) E\right]=0, \\
& \frac{\partial \beta}{\partial t}+\frac{\partial}{\partial x}\left[g b+\frac{g \kappa \omega_{0} E}{2 \sinh 2 \kappa h_{0}}\right]=0, \\
& \frac{\partial b}{\partial t}+\frac{\partial}{\partial x}\left[\beta h_{0}+\frac{1}{2} g \kappa E\right]=0 .
\end{aligned}
$$

These equations are in exact agreement with the results of WHITHAM [11] and for a detailed investigation of this set of equations we refer to that paper. 



\section{APPENDIX}

\section{Periodic progressive water waves of finite amplitude (Stokes waves)}

The exact formulation of the boundary value problem for irrotational water waves is as follows: within the incompressible fluid bounded by the bottom $y=-h_{0}$ and the free surface $y=\eta(x, t)$ we have a velocity potential $\Phi(x, y, t)$ defined by

$$
u=\frac{\partial \Phi}{\partial x}, \quad v=\frac{\partial \Phi}{\partial y},
$$

and satisfying the Laplace equation

$$
\Phi_{x x}+\Phi_{y y}=0 .
$$

The boundary condition at the bottom is: (See fig. 4)

$$
\left[\Phi_{y}\right]_{y=-h_{0}}=0 .
$$

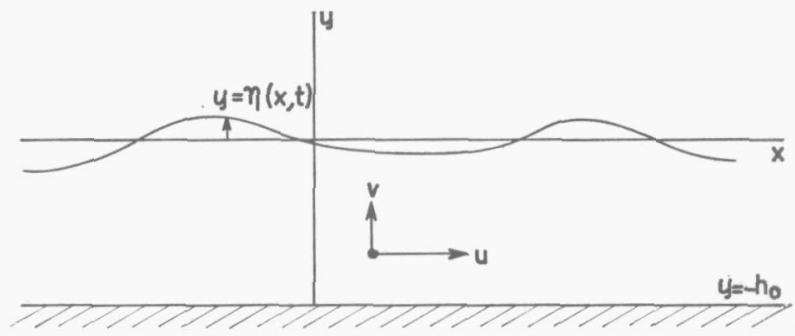

Fig. 4.

On the free surface $y=\eta(x, t)$ we have the kinematical condition

$$
\eta_{t}+\eta_{x}\left[\Phi_{x}\right]_{y=\eta(x, t)}-\left[\Phi_{y}\right]_{y=\eta(x, t)}=0,
$$

and the condition of constant pressure:

$$
g \eta+\left[\Phi_{t}+\frac{1}{2}\left(\Phi_{x}^{2}+\Phi_{y}^{2}\right)\right]_{y=\eta(x, t)}=\text { constant }(=\gamma) .
$$

A periodic wavetrain propagating with phase velocity $c$ in the direction of the positive $x$-axis is given by 


$$
\begin{aligned}
& u(x, y, t)=u(x-c t, y), \\
& v(x, y, t)=v(x-c t, y), \\
& \eta(x, t)=\eta(x-c t),
\end{aligned}
$$

where $u, v$ and $\eta$ are periodic functions of the phase function $\theta=x-c t$. Many authors starting with STOKES in 1847 have given solutions of equations (A.1) with boundary conditions (A.2), (A.3) and (A.4) in the form of a uniform progressive wavetrain of the form

$$
\begin{aligned}
& \Phi(x, y, t)=\Phi(x-c t, y), \\
& \eta(x, t)=\eta(x-c t) .
\end{aligned}
$$

with $\Phi$ and $\eta$ periodic in $\theta=x-c t$, for instance LAMB [18], Bowden [19], and WeHAUSEN [20]. The assumption of periodicity of $\Phi$ in $\theta$ does not allow a mean velocity $\beta$ in the $x$-direction. Such a mean velocity would give rise to a term $\beta x$ in $\Phi$ which is not periodic in $\theta$. In this Appendix a term $\beta x$ is added to the potential $\Phi$ and also the constant $\gamma$ occurring in the condition of constant pressure (A.4) is absorbed into $\Phi$ by adding a term $-\gamma t$. Hence we construct a solution of equation (A.1) with boundary conditions (A.2), (A.3) and (A.4) of the form

$$
\left.\begin{array}{l}
\Phi(x, y, t)=\beta x-\gamma t+\varphi(x-c t, y) \\
\eta(x, t)=\eta(x-c t),
\end{array}\right\}
$$

with $\varphi$ and $\eta$ bounded periodic functions of the phase $\theta=x-c t$. This general representation is also used by WHITHAM [11] who proceeds by applying an averaged Lagrangian principle in order to find the solution. We will derive the same results by a formal perturbation procedure.

Insertion of equations (A.5) into the boundary value problem for $\Phi(x, y, t)$ and $\eta(x, t)$ gives a boundary value problem involving $\varphi(\theta, y)$ and $\eta(\theta)$. The equation is:

$$
\varphi_{\theta \theta}+\varphi_{y y}=0 \quad \text { for } \quad-h_{0} \leqq y \leqq \eta(\theta),
$$

with boundary condition at the bottom $y=-h_{0}$ :

$$
\varphi_{y}=0,
$$

and on the free surface $y=\eta(\theta)$ :

$$
\begin{array}{ll}
(\beta-c) \eta_{\theta}+\eta_{\theta} \varphi_{\theta}-\varphi_{y}=0, & \text { on } y=\eta(\theta), \\
g \eta+(\beta-c) \varphi_{\theta}+\frac{1}{2}\left(\varphi_{\theta}^{2}+\varphi_{y}^{2}\right)=\gamma-\frac{1}{2} \beta^{2}, & \text { on } \gamma=\eta(\theta) .
\end{array}
$$


An exact solution of this problem is not available and all investigations of Stokes waves are concerned with finding series expansions of $\varphi(\theta, y)$ and $\eta(\theta)$ in powers of a small parameter $\varepsilon=\kappa a$, where $\kappa$ is the wavenumber per $2 \pi$ and $a$ is a measure for the amplitude of the wave. This means that we are looking for waves of moderately small amplitude and given wavelength $2 \pi / \kappa$.

Hence we suppose that the various functions and constants occurring in the boundary value problem for $\varphi(\theta, y)$ and $\eta(\theta)$ may be expanded as follows:

$$
\begin{aligned}
\varphi(\theta, y) & =\varepsilon \Phi_{1}(\theta, y)+\varepsilon^{2} \Phi_{2}(\theta, y)+\varepsilon^{3} \Phi_{3}(\theta, y)+\ldots \\
\eta(\theta) & =\varepsilon U_{1}(\theta)+\varepsilon^{2} U_{2}(\theta)+\varepsilon^{3} U_{3}(\theta)+\ldots \\
c & =\frac{\omega}{\kappa}=c_{0}+\varepsilon c_{1}+\varepsilon^{2} c_{2}+\varepsilon^{3} c_{3}+\ldots \\
\gamma & =\varepsilon^{2} \gamma_{0}+\varepsilon^{3} \gamma_{1}+\ldots \\
\beta & =\varepsilon^{2} \beta_{0}+\varepsilon^{3} \beta_{1}+\ldots
\end{aligned}
$$

The leading terms in the series for $\varphi(\theta, y)$ and $\eta(\theta)$ are assumed to be of order $\varepsilon$ corresponding to linear waves of infinitesimal amplitude. Furthermore the constants $\beta$ and $\gamma$, being absent in linear theory, are assumed to be of order $\varepsilon^{2}$ and hence only play a part in the higher order perturbation terms accounting for the non-linear effects.

From equation (A.6) it is seen that all functions $\Phi_{i}(\theta, y)$ satisfy the equation:

$$
\Phi_{i \theta \theta}+\Phi_{i y y}=0, \quad i=1,2,3, \ldots,
$$

with boundary condition at the bottom $y=-h_{0}$ :

$$
\Phi_{i y}=0, \quad i=1,2,3, \ldots
$$

Free surface conditions (A.8) and (A.9) are expanded now in powers of $\varepsilon$ and yield the following conditions for the first order terms $\Phi_{1}(\theta, y)$ and $U_{1}(\theta)$ to be satisfied on the undisturbed free surface $y=0$ :

$$
\begin{aligned}
& c_{0} U_{1 \theta}+\Phi_{1 y}=0, \\
& g U_{1}-c_{0} \Phi_{1 \theta}=0 .
\end{aligned}
$$

The second order terms $\Phi_{2}(\theta, y)$ and $U_{2}(\theta)$ satisfy on $y=0$ : 


$$
\begin{aligned}
& c_{0} U_{2 \theta}+\Phi_{2 y}=-c_{1} U_{1 \theta}+\Phi_{1 \theta} U_{1 \theta}-U_{1} \Phi_{1 y y}, \\
& g U_{2}-c_{0} \Phi_{2 \theta}=c_{1} \Phi_{1 \theta}+c_{0} U_{1} \Phi_{1 \theta y}-\frac{1}{2}\left(\Phi_{1 \theta}^{2}+\Phi_{1 y}^{2}\right)+\gamma_{0} .
\end{aligned}
$$

The third order terms $\Phi_{3}(\theta, y)$ and $U_{3}(\theta)$ satisfy on $y=0$ :

$$
\begin{gathered}
c_{0} U_{3 \theta}+\Phi_{3 y}=-c_{1} U_{2 \theta}+\left(\beta_{0}-c_{2}\right) U_{1 \theta}-U_{1} \Phi_{2 y y}-U_{2} \Phi_{1 y y}+ \\
-\frac{1}{2} U_{1}^{2} \Phi_{1 y y y}+U_{2 \theta} \Phi_{1 \theta}+U_{1} U_{1 \theta} \Phi_{1 \theta y}+U_{1 \theta} \Phi_{2 \theta}, \\
g U_{3}-c_{0} \Phi_{3 \theta}=c_{0} U_{1} \Phi_{2 \theta y}+\frac{1}{2} c_{0} U_{1}^{2} \Phi_{1 \theta y y}+c_{0} U_{2} \Phi_{1 \theta y}+ \\
-\Phi_{1 \theta}\left(U_{1} \Phi_{1 \theta y}+\Phi_{2 \theta}\right)-\Phi_{1 y}\left(U_{1} \Phi_{1 y y}+\Phi_{2 y}\right)+ \\
+\gamma_{1}+c_{1}\left(\Phi_{2 \theta}+U_{1} \Phi_{1 \theta y}\right)-\left(\beta_{0}-c_{2}\right) \Phi_{1 \theta} .
\end{gathered}
$$

The first order boundary value problem for $\Phi_{1}(\theta, y)$ and $U_{1}(\theta)$ is solved by:

$$
\begin{aligned}
\varepsilon \Phi_{1}(\theta, y) & =A_{1} \cosh \left[\kappa\left(h_{0}+y\right)\right] \sin \kappa \theta, \\
\varepsilon U_{1}(\theta) & =a \cos \kappa \theta .
\end{aligned}
$$

The unknown constants $A_{1}$ and $c_{0}$ are expressed in terms of $\kappa$ and $a$ by substitution of (A.16) and (A.17) into the free surface conditions for $\Phi_{1}$ and $U_{1}$. This yields:

$$
A_{1}=\frac{a c_{0}}{\sinh \kappa h_{0}}
$$

and the linear dispersion relation:

$$
c_{0}^{2}(\kappa)=\frac{g}{\kappa} \operatorname{tgh} \kappa h_{0} .
$$

Free surface conditions (A.12) and (A.13) for $\Phi_{2}(\theta, y)$ and $U_{2}(\theta)$ become respectively:

$$
\begin{aligned}
& c_{0} U_{2 \theta}+\Phi_{2 y}=c_{1} \sin \kappa \theta-c_{0} \operatorname{cotgh} \kappa h_{0} \sin 2 \kappa \theta, \\
& g U_{2}-c_{0} \Phi_{2 \theta}=c_{0} c_{1} \operatorname{cotgh} \kappa h_{0} \cos \kappa \theta+\gamma_{0}+\frac{1}{4} c_{0}^{2}+ \\
& \quad-\frac{1}{4} c_{0}^{2} \operatorname{cotgh}^{2} \kappa h_{0}+\frac{1}{4} c_{0}^{2}\left(3-\operatorname{cotgh}^{2} \kappa h_{0}\right) \cos 2 \kappa \theta .
\end{aligned}
$$

Elimination of $U_{2}(\theta)$ gives one condition for $\Phi_{2}(\theta, y)$ to be satisfied on $y=0$ : 


$$
\begin{gathered}
\frac{c_{0}^{2}}{g} \Phi_{2 \theta \theta}+\Phi_{2 y}=2 c_{1} \sin \kappa \theta-c_{0} \operatorname{cotgh} \kappa h_{0} \sin 2 \kappa \theta+ \\
+\frac{c_{0}^{3} \kappa}{g}\left[1-\frac{1}{2 \sinh ^{2} \kappa h_{0}}\right] \sin 2 \kappa \theta .
\end{gathered}
$$

The general solution of equation (A.10) satisfying boundary condition (A.11) and having period $2 \pi / \kappa$ in $p$, is given by

$$
\Phi_{2}(\theta, y)=\alpha_{0}+\sum_{j=1}^{\infty} \alpha_{j} \cosh \left[j \kappa\left(h_{0}+y\right)\right] \sin j \kappa \theta .
$$

Calculating the unknown coefficients $\alpha_{j}$ by substitution of (A.23) into free surface condition (A.22) it is seen that the term with $j=1$ automatically satisfies the homogeneous boundary condition:

$$
\frac{c_{0}^{2}}{g} \Phi_{2 \theta \theta}+\Phi_{2 y}=0 \quad \text { on } \quad y=0
$$

as a result of equation (A.19). Hence the term with $\sin \kappa \theta$ in the righthand side of condition (A.22) can never be produced by the periodic solution (A.23). So we put $c_{1}=0$. Solutions of the homogeneous problem of the form

$$
\alpha_{1} \cosh \left[\kappa\left(h_{0}+y\right)\right] \sin \kappa \theta,
$$

may be added to $\Phi_{2}(\theta, y)$ but defining the amplitude $a$ by fixing $A_{1}=a c_{0} / \sinh \kappa h_{0}$ as the coefficient of the first term $\cosh \left[\kappa\left(h_{0}+y\right)\right] \sin \kappa \theta$ in the expansion of $\varphi(\theta, y)$, then terms such as (A.24) need not be taken into account in the higher order approximations $\Phi_{2}(\theta, y), \Phi_{3}(\theta, y)$, etc.

Thus the solution of the second order boundary value problem is

$$
\varepsilon^{2} \Phi_{2}(\theta, y)=A_{2} \cosh \left[2 \kappa\left(h_{0}+y\right)\right] \sin 2 \kappa \theta
$$

where $A_{2}$ is found by satisfying condition (A.22):

$$
A_{2}=\frac{3}{8} \frac{a^{2} \omega_{0}}{\sinh ^{4} \kappa h_{0}}=O\left(\varepsilon^{2}\right)
$$

The corresponding second approximation $\varepsilon^{2} U_{2}(\theta)$ to the free surface elevation is calculated from equation (A.21):

$$
\varepsilon^{2} U_{2}(\theta)=b+a_{2} \cos 2 \kappa \theta,
$$


where the first approximation $b$ to the mean waveheight is given by

$$
b=\frac{\varepsilon^{2} \gamma_{0}}{g}-\frac{\kappa a^{2}}{2 \sinh 2 \kappa h_{0}}=O\left(\varepsilon^{2}\right)
$$

and the constant $a_{2}$ is determined by

$$
a_{2}=\frac{1}{4} \kappa a^{2} \frac{3-\operatorname{tgh}^{2} \kappa h_{0}}{\operatorname{tgh}^{3} \kappa h_{0}}=O\left(\varepsilon^{2}\right) .
$$

In order to obtain the second order term $\varepsilon^{2} c_{2}$ in the dispersion relation we have to investigate the secular terms, viz. terms containing $\sin \kappa \theta$, in the boundary condition of the third order problem. By substitution of the first and second order results into equations (A.14) and (A.15) the third order free surface conditions are found to be as follows:

$$
\begin{aligned}
& c_{0} U_{3 \theta}+\Phi_{3 y}=\mu_{1} \sin \kappa \theta+\mu_{3} \sin 3 \kappa \theta, \\
& g U_{3}-c_{0} \Phi_{3 \theta}=\sigma_{1} \cos \kappa \theta+\sigma_{3} \cos 3 \kappa \theta+\gamma_{1},
\end{aligned}
$$

with constants $\mu_{1}$ and $\sigma_{1}$ given by

$$
\begin{aligned}
\varepsilon^{3} \mu_{1} & =-\kappa^{3} a^{3}\left(\beta_{0}-c_{2}\right)-\kappa^{2} a A_{2} \cosh 2 \kappa h_{0}-\frac{3}{8} a^{2} \kappa^{3} A_{1} \sinh \kappa h_{0}+ \\
& -\kappa^{2} A_{1}\left(\frac{1}{2} a_{2}+b\right) \cosh \kappa h_{0}, \\
\varepsilon^{3} \sigma_{1} & =\left(2 a c_{0} A_{2} \kappa^{2}-\frac{1}{2} a A_{1}^{2} \kappa^{3}\right) \sinh 2 \kappa h_{0}+\left[\frac{3}{8} c_{0} a^{2} A_{1} \kappa^{3}-\left(\beta_{0}-c_{2}\right) a^{2} \kappa^{3} A_{1}\right] \cosh \kappa h_{0}+ \\
& +\left(b c_{0} A_{1} \kappa^{2}+\frac{1}{2} c_{0} a_{2} A_{1} \kappa^{2}\right) \sinh \kappa h_{0}-A_{1} A_{2} \kappa^{2} \cosh 3 \kappa h_{0} .
\end{aligned}
$$

The constants $\mu_{3}$ and $\sigma_{3}$ will not be needed because only terms with $\sin \kappa \theta$ and $\cos \kappa \theta$ give rise to secular terms. Elimination of $U_{3}(\theta)$ gives a single free surface condition for $\Phi_{3}(\theta, y)$ to be satisfied on $y=0$ :

$$
\frac{c_{0}^{2}}{g} \Phi_{3 \theta \theta}+\Phi_{3 y}=\left[\mu_{1}+\frac{\sigma_{1} \kappa c_{0}}{g}\right] \sin \kappa \theta+\left[\mu_{3}+\frac{3 \kappa c_{0} \sigma_{3}}{g}\right] \sin 3 \kappa \theta .
$$

By a similar reasoning as before it follows that no periodic solution can satisfy this condition with the term containing $\sin \kappa \theta$ in the righthand side and hence we must have:

$$
\mu_{1}+\frac{\sigma_{1} \kappa c_{0}}{g}=0
$$


After considerable manipulation this relation transforms into:

$$
\varepsilon^{2}\left(c_{2}-\beta_{0}\right)=\frac{g b}{2 c_{0} \cosh ^{2} \kappa h_{0}}+\frac{1}{2} g a^{2} \kappa \frac{D_{0}}{c_{0}},
$$

with the abbreviation:

$$
D_{0}=\frac{9 \operatorname{tgh}^{4} \kappa h_{0}-10 \operatorname{tgh}^{2} \kappa h_{0}+9}{8 \operatorname{tgh}^{3} \kappa h_{0}} .
$$

Summarizing we have obtained the following results up to the second order of approximation:

$$
\begin{gathered}
\Phi(x, y, t)=\beta x-\gamma t+A_{1} \cosh \left[\kappa\left(h_{0}+y\right)\right] \sin \kappa \theta+ \\
+A_{2} \cosh \left[2 \kappa\left(h_{0}+y\right)\right] \sin 2 \kappa \theta+O\left(\varepsilon^{3}\right), \\
\eta(\theta)=b+a \cos \kappa \theta+a_{2} \cos 2 \kappa \theta+O\left(\varepsilon^{3}\right),
\end{gathered}
$$

with constants $A_{1}, A_{2}$ and $a_{2}$ given by

$$
\begin{aligned}
& A_{1}=\frac{a c_{0}}{\sinh \kappa h_{0}}, \\
& A_{2}=\frac{3}{8} \frac{a^{2} \omega_{0}}{\sinh ^{4} \kappa h_{0}}, \\
& a_{2}=\frac{1}{4} \kappa a^{2} \frac{3-\operatorname{tgh}^{2} \kappa h_{0}}{\operatorname{tgh}^{3} \kappa h_{0}} .
\end{aligned}
$$

Furthermore we have the dispersion relation

where

$$
\omega(\kappa)=c \kappa=\omega_{0}(\kappa)+\beta \kappa+\frac{g b \kappa^{2}}{2 \omega_{0} \cosh ^{2} \kappa h_{0}}+\frac{1}{2} g \kappa^{3} a^{2} \frac{D_{0}}{\omega_{0}}+O\left(\varepsilon^{3}\right),
$$

$$
\omega_{0}^{2}(\kappa)=g \kappa \operatorname{tgh} \kappa h_{0},
$$

and also a ,pseudo-dispersion relation':

$$
g b=\gamma-\frac{g \kappa a^{2}}{2 \sinh 2 \kappa h_{0}}+O\left(\varepsilon^{3}\right)
$$


The uniform wavetrain solution for Stokes waves depends on the six parameters $\kappa, \omega, a, b, \beta$ and $\gamma$ with two dispersion relations connecting them and hence four of them can be chosen independently. 


\section{REFERENCES}

1. BRoER, L. J. F.

On the propagation of energy in linear conservative waves, Appl. Sci. Res., vol. A2, pp. 329-344, 1950.

2. LighthiLL, M. J.

Group velocity, J. Maths. Appl., vol. 1, pp. 1-28, 1965.

3. ECKART, C.

The approximate solution of one-dimensional wave equations, Rev. Mod. Phys., vol. 20, no. 2, pp. 399-417, 1948.

4. Brillouin, L. \& Sommerfeld, A.

Wave propagation and group velocity, Academic Press, New York, 1960.

5. JeFFreYs, H. \& JeFFreYs, B. S.

Methods of mathematical physics, Cambridge Univ. Press, 1946.

6. LewIS, R. M.

Asymptotic methods for the solution of dispersive hyperbolic equations. In: Asymptotic solutions of differential equations and their applications, C. H. WILcox, ed., University of Wisconsin Symposium Proceedings, pp. 53-107, Wiley, New York, 1964.

7. Bleistein, N. \& Lewis, R. M.

Space-time diffraction for dispersive hyperbolic equations, SIAM J. Appl. Math., vol. 14, no. $6, \mathrm{pp} .1454-1470,1966$.

8. BOERSMA, J.

Uniform asymptotic solutions of discontinuous initial value problems for dispersive hyperbolic equations, Quart. Appl. Math., vol. 25, no. 1, pp. 31-44, 1967.

9. Whiтнам, G. B.

Non-linear dispersive waves, Proc. Roy. Soc. A, vol. 283, pp. 238-261, 1965.

10. Whitham, G. B.

A general approach to linear and non-linear dispersive waves using a Lagrangian, J. Fluid Mech., vol. 22, pp. 273-283, 1965.

11. Whitham, G. B.

Non-linear dispersion of water waves, J. Fluid Mech., vol. 27, pp. 399-412, 1967.

12. Whitham, G. B.

Variational methods and applications to water waves, Proc. Roy. Soc. A, vol. 299, pp. 6-25, 1967.

13. Lighthill, M. J.

Contributions to the theory of waves in non-linear dispersive systems, J. Maths. Appl., vol. 1, pp. 269-306, 1965.

14. LighthILL, M. J.

Some special cases treated by the Whitham theory, Proc. Roy. Soc. A, vol. 299, pp. 28-53, 1967.

15. LUKE, J. C.

A perturbation method for non-linear dispersive wave problems, Proc. Roy. Soc. A, vol. 292, pp. 403-412, 1966. 
16. KoRTEWEG, D. J. \& DE VRIES, G.

On the change of form of lon g waves advancing in a rectangular canal, Phil. Mag. (5), vol. 39, pp. 422-443, 1895.

17. MiNORSKY, N.

Non-linear oscillations, Van Nostrand, Princeton, 1962.

18. LAMB, $\mathrm{H}$.

Hydrodynamics, 6th ed., Cambridge Univ. Press, 1932. (Dover Press, New York, 1945).

19. BOWDEN, K. F.

Some observations of waves and other fluctuations in a tidal current. Appendix: Surface waves of finite amplitude in water of finite depth. Proc. Roy. Soc. A, vol. 192, p. 403, 1948.

20. Wehausen, J. V. \& Lattone, E. V.

Surface waves, Handbuch der Physik, ed. S. Flügge, Band IX, Strömungsmechanik III, Springer Verlag, Berlin, 1960. 


\section{SAMENVATTING}

In dit proefschrift wordt de voortplanting van een drietal soorten niet-lineaire dispersieve watergolven bestudeerd, te weten cnoïde golven, Boussinesq-golven en Stokes-golven.

De inleiding is gewijd aan een algemene beschouwing over dispersieve golfverschijnselen. Wij voeren het begrip ,langzaam veranderlijke golf' in, dat wil zeggen een golf die plaatselijk als nagenoeg periodiek mag worden beschouwd. Voor een dergelijke golf veranderen grootheden zoals golfgetal, frequentie, amplitude, enz. slechts zeer weinig binnen een afstand van enkele golflengten of gedurende enkele perioden. Indien een dispersief systeem een willekeurige beginverstoring krijgt, dan zal in het algemeen deze verstoring zich na lange tijd ontwikkelen (,dispergeren') tot een langzaam veranderlijke golf. Asymptotische oplossingen van bovengenoemde watergolf-problemen in de vorm van langzaam veranderlijke golven zijn het onderwerp van dit proefschrift.

$\mathrm{Na}$ een kort overzicht van lineaire golven en van de methode van WHITHAM voor niet-lineaire conservatieve golfverschijnselen wordt de inleiding besloten met een uiteenzetting over een algemene asymptotische representatie van langzaam veranderlijke golven, die het uitgangspunt vormt voor de berekeningen in de daaropvolgende hoofdstukken. De asymptotiek wordt betrokken op een grote parameter $K$, waarbij $K^{-1}$ een maat is voor de langzame variaties van golfgetal, frequentie, amplitude, enz.. Men beschouwt nu een gestrekt coördinatenstelsel waarin iedere lengte-eenheid en iedere tijdseenheid een groot aantal (van de orde van grootte $K$ ) golflengten, respectievelijk perioden bevat. In een dergelijk coördinatenstelsel kunnen lijnen van constante fase of golffronten gedefinieerd worden als lijnen waarlangs de normale afgeleide van de golffunctie van de orde van grootte $K$ is, terwijl de tangentiële afgeleide van de orde van grootte 1 is. Een asymptotische voorstelling van een langzaam veranderlijke golf, die op deze definitie van golffronten is gebaseerd, kan vervolgens in de partiële differentiaalvergelijking voor de gezochte golffunctie worden gesubstitueerd.

Nadat de coëfficiënten van de verschillende machten van $K$ gelijk aan nul zijn gesteld, blijkt de hoofdterm van de asymptotische reeks te voldoen aan de gewone differentiaalvergelijking voor de uniforme lopende golfoplossing van het probleem, met dien verstande dat de constante parameters in die uniforme lopende golfoplossing nu langzaam veranderlijke functies van de coördinaten zijn. Voor deze functies, die de veranderingen op grote schaal van de golf beheersen, worden differentiaalvergelijkingen afgeleid. Allereerst voldoen zij aan de zgn. dispersierelatie, die een betrekking 
geeft tussen de diverse constante parameters van de uniforme lopende golf en die ook geldig blijkt te zijn voor de langzaam veranderlijke parameters. Verder geldt er een betrekking tussen golfgetal en frequentie.

De overige relaties tussen de langzaam veranderlijke parameters van het probleem volgen uit de eis dat de tweede term van de asymptotische ontwikkeling begrensd moet zijn. Dit leidt tot een aantal integraalrelaties voor de hoofdtermen. Voor de lange golven op ondiep water, die wij in de hoofdstukken I en II behandelen, wordt vervolgens aangetoond dat deze integraalrelaties ook verkregen kunnen worden door enkele behoudswetten van het probleem op geschikte wijze te middelen over één periode.

De aldus verkregen vergelijkingen voor de langzaam veranderlijke parameters worden verder vereenvoudigd door een asymptotische ontwikkeling naar machten van de klein veronderstelde amplitude/diepte-verhouding $\varepsilon$. Hierbij brengen wij slechts de laagste orde niet-lineaire effecten in rekening. In het geval van de cnoïde golven en van de Boussinesq-golven ontstaat er na transformatie in de karakteristieke vorm een stelsel van twee partiële differentiaalvergelijkingen voor golfgetal en amplitude, dat equivalent is met de vergelijkingen voor de niet-stationaire beweging van een samendrukbaar gas. Voor het beginwaardenprobleem voor langzaam veranderlijke golven wordt van deze vergelijkingen een asymptotische oplossing gegeven. In het derde hoofdstuk, waarin Stokes golven worden behandeld, blijken de resultaten overeen te stemmen met die van WhIтHAM. In de appendix geven wij een afleiding van de uniforme, periodieke Stokes-golf, ontwikkeld naar machten van de kleine amplitude/golflengteverhouding. 


\section{STELLINGEN}

I.

Analoog aan de cnoïde golven en de Boussinesq golven die in hoofdstuk I, respectievelijk hoofdstuk II van dit proefschrift worden behandeld, kan voor de Stokes golven in hoofdstuk III het stelsel vergelijkingen voor golfgetal, amplitude, gemiddelde snelheid en gemiddelde golfhoogte worden gereduceerd tot een stelsel van twee vergelijkingen voor golfgetal en amplitude.

II.

Voor de in dit proefschrift behandelde watergolfproblemen is het ook mogelijk asymptotische oplossingen in de vorm van langzaam veranderlijke golven te vinden, indien de ongestoorde diepte een langzaam veranderlijke functie van de coördinaten is.

III.

De Poincaré-Lighthill-Kuo-methode kan in enigszins aangepaste vorm worden toegepast om uniform geldige benaderingen te verkrijgen van de onsamendrukbare, twee-dimensionale stroming om een dun draagvlak met afgeronde voorkant.

H. S. Tsien, The Poincaré-Lighthill-Kuo-method, Advances in Applied Mechanics, vol. 4, pp. 281-349, 1956.

H. W. Hoogstraten, Uniformly Valid Approximations in Two-dimensional Subsonic Thin Airfoil Theory, J. Engineering Math., vol. 1, pp. 51-65, 1967.

\section{IV.}

Het quasi-stationaire probleem van een vlakke watergolf, die schuin invalt op een langzaam oplopend strand, kan in het kader van de gelineariseerde theorie van golven op ondiep water asymptotisch worden opgelost door gebruik te maken van de volgende representatie van de golfhoogte $\eta(x, y)$ :

$$
\eta(x, y) \sim\left\{A_{0}(x, y)+i \varepsilon A_{1}(x, y)+\ldots\right\} \exp \left\{i \varepsilon^{-1} S(x, y)\right\},
$$

waarin $x$ en $y$ de horizontale coördinaten voorstellen, $A(x, y)$ een amplitude- en $S(x, y)$ een fase-functie is, en $\varepsilon$ een kleine parameter is, die bepaald wordt door de kleine helling van het strand.

\section{V.}

De gelineariseerde, twee-dimensionale stroming met cavitatie van een onsamendrukbare vloeistof om een vlakke plaat onder kleine invalshoek kan eenvoudig worden berekend met behulp van conforme afbeeldingen. 

VI.

Volgens Song is voor het pulseren van een geventileerde caviteit de aanwezigheid van een vrij oppervlak essentieel. Latere onderzoekingen hebben aangetoond dat deze bewering onjuist is.

C. S. Song, Pulsation of Ventilated Cavities, J. Ship Res., vol. 5, no. 4, pp. 8-20, 1962.

VII.

Teneinde een inzicht te krijgen in de boeggolf van een schip met afgeronde voorkant, verdient het aanbeveling de stroming met vrij oppervlak langs een oneindig lange, verticale parabolische cilinder te onderzoeken.

VIII.

Van de niet-lineaire partiële differentiaalvergelijking

$$
u_{t t}-u_{x x}+K^{2} V^{\prime}(u)=0, \quad(K \gg 1)
$$

kan voor een langzaam veranderlijke golfoplossing op eenvoudige wijze een vergelijking voor de fase-functie $S(x, t)$ gevonden worden door substitutie van de plaatselijk geldige asymptotische representatie:

$$
u(x, t) \sim A(x, t) \Phi\{K S(x, t)\},
$$

waarin $A(x, t)$ een amplitude-functie en $\Phi$ een functie van de fase $K S(x, t)$ is.

\section{IX.}

De mogelijkheid tot snelle publikatie van artikelen, zoals deze door het Journal of Engineering Mathematics wordt geboden, kan stimulerend werken op diegenen in Nederland, die zich met de toepassing van wiskundige methoden op technische problemen bezighouden.

$\mathrm{X}$.

Het is nuttig om studenten in de toegepaste wiskunde in een zo vroeg mogelijk stadium van hun studie zelfstandig te laten werken aan de wiskundige formulering en oplossing van problemen uit de praktijk. 
